University of Louisville

ThinkIR: The University of Louisville's Institutional Repository

\title{
Sleep/wake activity and energy expenditure in overweight and obese obstructive sleep apnea patients : a pre- and post-CPAP comparison.
}

\author{
Pamela A. McCullough
}

University of Louisville

Follow this and additional works at: https://ir.library.louisville.edu/etd

\section{Recommended Citation}

McCullough, Pamela A., "Sleep/wake activity and energy expenditure in overweight and obese obstructive sleep apnea patients : a pre- and post-CPAP comparison." (2011). Electronic Theses and Dissertations. Paper 943.

https://doi.org/10.18297/etd/943

This Doctoral Dissertation is brought to you for free and open access by ThinkIR: The University of Louisville's Institutional Repository. It has been accepted for inclusion in Electronic Theses and Dissertations by an authorized administrator of ThinkIR: The University of Louisville's Institutional Repository. This title appears here courtesy of the author, who has retained all other copyrights. For more information, please contact thinkir@louisville.edu. 


\title{
SLEEP/WAKE ACTIVITY AND ENERGY EXPENDITURE IN OVERWEIGHT AND OBESE OBSTRUCTIVE SLEEP APNEA PATIENTS: A PRE- AND POST-CPAP COMPARISON
}

\author{
By \\ Pamela A. McCullough \\ B.S.N., University of Louisville, 1991 \\ M.S.N., University of Louisville, 1997

\begin{abstract}
A Dissertation
Submitted to the Faculty of the Graduate School of the University of Louisville in Partial Fulfillment of the Requirements for the Degree of
\end{abstract}

Doctor of Philosophy

School of Nursing University of Louisville Louisville, Kentucky

August 2011 
Copyright 2011 by Pamela A. McCullough

All rights reserved 

SLEEP/WAKE ACTIVITY AND ENERGY EXPENDITURE IN OVERWEIGHT AND OBESE OBSTRUCTIVE SLEEP APNEA PATIENTS: A PRE-AND POST-CPAP COMPARISON

By

Patricia A. McCullough

B.S.N., University of Louisville, 1991

M.S.N., University of Louisville, 1997

A Dissertation Approved on

July 27, 2011

By the following Dissertation Committee:

Barbara Speck

Dissertation Chair

David Winslow

John Myers

Diane Chlebowy

Rosalie Mainous 


\section{ACKNOWLEDGMENTS}

I would like to thank my major professor, Dr. Barbara Speck, for her guidance, patience, and encouragement. To Dr. Speck and my committee members, Dr. Rosalie Mainous, Dr. Diane Chlebowy, Dr. John Myers, and Dr. David Winslow, I would like to express sincere gratitude for sharing their knowledge, expertise, and wisdom throughout this journey. I would also like to extend deepest appreciation to the physicians and staff of Kentucky Research Group, Chest Medicine Associates, and Sleep Medicine Specialists; without their kindness, support, and daily affirmations I would have never seen this daunting challenge and commitment to completion. I would also like to acknowledge my family and friends, for their respect and admiration of higher education and my pursuit of a doctoral degree. Last, but certainly not least, very special recognition goes to Elizabeth McReynolds and my children, Caroline and Ethan, for providing unconditional love and devotion throughout this life-changing endeavor. They surrounded me with joy and filled me with inner peace through the best and worst of times. I love you all so very much. 


\begin{abstract}
SLEEP/WAKE ACTIVITY AND ENERGY EXPENDITURE IN OVERWEIGHT AND OBESE OBSTRUCTIVE SLEEP APNEA PATIENTS: A PRE- AND POST-CPAP COMPARISON
\end{abstract}

Pamela A. McCullough

July 27,2011

Overweight and obesity is a major contributing factor in an estimated $70 \%$ of all obstructive sleep apnea (OSA) cases. Approximately five percent of the adult population has OSA, and the numbers continue to soar with the rising prevalence of obesity. As a primary therapy, continuous positive airway pressure (CPAP) has demonstrated improvement in many comorbities associated with both OSA and obesity. Although anecdotal and clinical references support increased energy, activity, and weight loss as a potential benefit of CPAP treatment, there remains little evidence to endorse CPAP as a significant weight-reduction measure for overweight and obese OSA patients. The purpose of this study was to examine sleep/wake activity and energy expenditure, preand post-CPAP treatment, in adult overweight and obese patients with OSA. A prospective, observational, longitudinal study design was employed to assess 24-hour actigraphic measures of sleep/wake activity and energy expenditure prior and subsequent to CPAP therapy. Analysis of variance (ANOVA) using repeated measures was performed to identify overall differences between pre-CPAP and one week post-CPAP and pre-CPAP and one month and post-CPAP sleep activity, wake activity, and 24-hour 
energy expenditure. Sixty-nine subjects were consented, with a total of 35 subjects completing the study. Data analyses revealed statistically significant mean differences in sleep activity, wake activity, and energy expenditure from pre-CPAP to post-CPAP at one week and one month. At baseline, and continuing through one week post-CPAP and one month post-CPAP, the CPAP compliant group demonstrated less sleep activity, more wake activity, and expended more energy than the CPAP noncompliant group. This study concluded that CPAP use is a statistically significant factor affecting sleep activity time, wake activity time, and energy expenditure. Regardless of CPAP compliance and length of use, the entire post-CPAP group demonstrated more sleep activity, had less wake activity, and burned fewer calories. From a clinical perspective, the results of this study do not support the use of CPAP as a potential weight loss measure in overweight and obese OSA patients, and emphasize the need for the inclusion of behavioral weight management and weight loss strategies in an at-risk population for comorbid illnesses. 


\section{TABLE OF CONTENTS}

PAGE

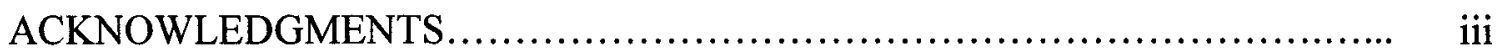

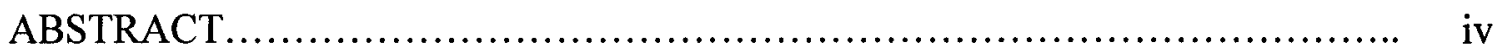

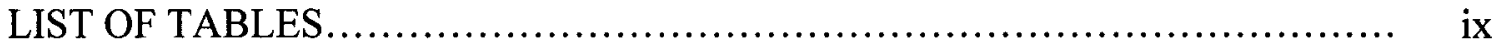

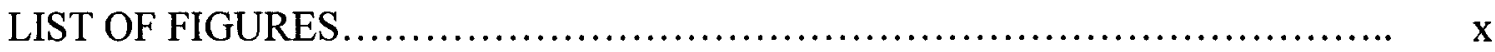

\section{CHAPTER}

I. RESEARCH PROBLEM....................................................

Background and Significance............................................ $\quad 1$

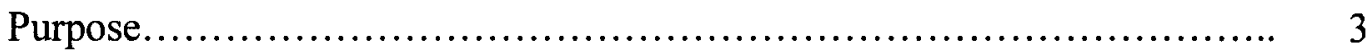

Conceptual Framework ................................................. 4

Hypotheses.......................................................... 12

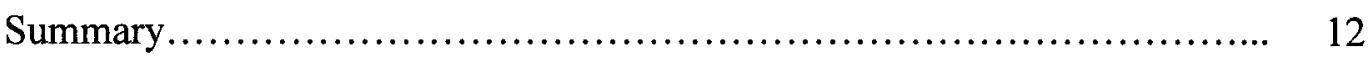

II. LITERATURE REVIEW ............................................ 14

Introduction..................................................... 14

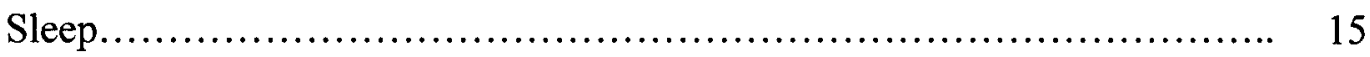

Obstructive Sleep Apnea............................................... 16

Continuous Positive Airway Pressure....................................... 29

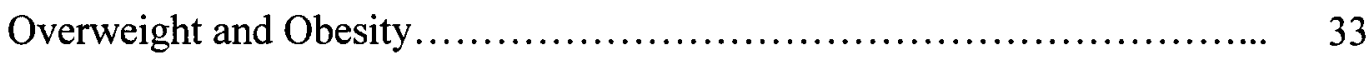

Sleep, Wake, and Energy Expenditure................................... 38

Actigraphy ......................................................... 43

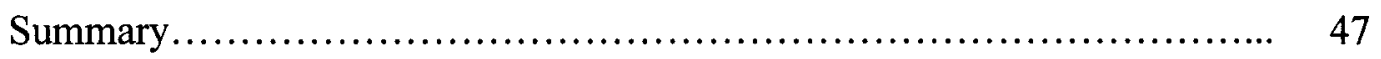


III. METHODS ................................................................. 52

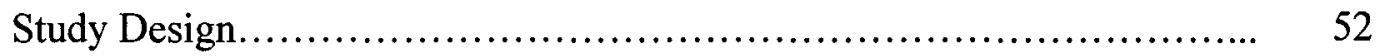

Sample and Setting................................................... 53

Human Subjects Considerations........................................ 55

Measurement............................................................ 56

Data Collection Procedures............................................... 63

Data Management Procedures........................................... 67

Statistical Analysis..................................................... $\quad 68$

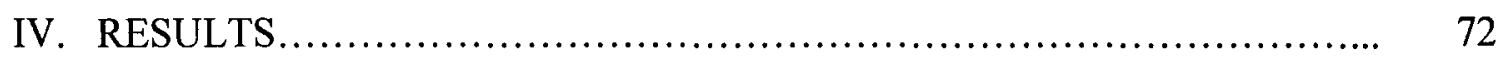

Descriptive Statistics........................................... 72

Repeated Measures Analysis of Variance............................... $\quad 82$

Paired $t$-tests.......................................................... 87

V. DISCUSSION .......................................................... 92

Introduction........................................................ 92

Findings......................................................... 93

Conclusions............................................................ 97

Implications...................................................... 99

Limitations.............................................................. 100

Recommendations.................................................... 101

REFERENCES...................................................... 103

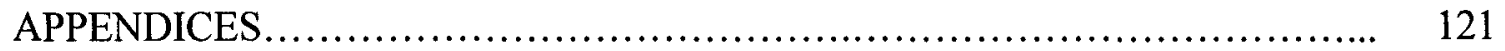

A. Human Subjects Protection Program Office Approval Letters.......... 121

B. Actical Report................................................ 127 
C. Actiwatch Report................................................ 128

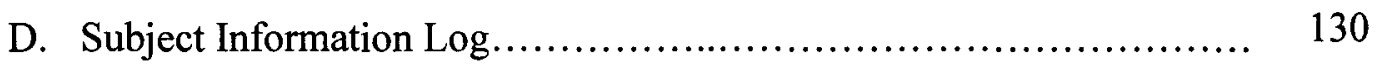

E. Obstructive Sleep Apnea Polysomnography Data.................... 131

F. CPAP Titration Polysomnography Data.................................. 132

G. Obstructive Sleep Apnea Polysomnography Report.................. 133

H. CPAP Titration Polysomnography Report............................. 136

I. CPAP Compliance Report........................................... 138

J. Visit Schedule of Procedures...................................... 140

K. Data Collection Forms............................................. 141

CURRICULUM VITAE .................................................... 153 


\section{LIST OF FIGURES}

FIGURES

PAGE

1. Conceptual Framework Schematic Model....................................... 5

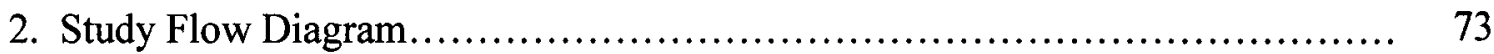

3. Sleep Activity Over Time Stratified by Compliance............................ 84

4. Wake Activity Over Time Stratified by Compliance............................. 85

5. Energy Expenditure Over Time Stratified by Compliance........................ 86 


\section{CHAPTER I}

\section{RESEARCH PROBLEM}

\section{Background and Significance}

Obstructive sleep apnea (OSA) is a sleep disorder that disproportionately affects individuals who are overweight or obese. Approximately five percent of the adult population has OSA, with overweight and obesity a major contributing factor in an estimated $70 \%$ of these cases (Young, Peppard, \& Gottlieb, 2002). Diagnostically, OSA is characterized by repeated upper airway collapse during sleep with subsequent reduction or cessation of airflow for greater than 10 seconds, occurring at least five times per hour of sleep. Either complete or partial obstruction of the upper airway and airflow results in decreased oxygenation and arousal from sleep. The most common cause of apnea is obesity and associated adipose tissue deposits within the neck, compressing and narrowing the upper airway, which collapses when the dilator muscle tone is reduced during sleep (Dempsey, Veasey, Morgan, \& O’Donnell, 2010). OSA is often suspected when snoring, gasping or choking episodes, disrupted sleep, morning headache, and daytime sleepiness is reported (Guilleminault \& Bassiri, 2005). Furthermore, sleep apnea has been linked to increased risk of hypertension, heart attack, stroke, heart failure, insulin resistance, depression, fatigue, and sleepiness with related occupational and driving accidents (Kapur et al., 1999; Phillips \& Kryger, 2005; Punjabi \& Beamer, 2005; Won \& Robert, 2007; Young, Peppard, \& Gottlieb, 2002). 
Polysomnography (PSG) is the standard diagnostic testing procedure used to confirm OSA, which measures physiologic parameters during sleep. Traditionally, treatment of OSA depends on the severity, determined from PSG as a calculated apneahypopnea index (AHI) or respiratory disturbance index (RDI). Conservative treatment is often the first line of therapy, with weight loss a primary recommendation. Weight loss has been shown effective in reducing apneic events, particularly in cases where weight gain coincided with worsening of apnea and related symptoms (Peppard, Young, Palta, \& Skatrud, 2000; Phillips \& Kryger, 2005). Lifestyle and behavioral modifications and sleep hygiene are typical conservative treatment recommendations and often include weight loss strategies, such as increased physical activity and reduced calorie diets. According to the Cochrane Database of Systematic Reviews, the success and value of these first line measures has not been established (Shneerson \& Wright, 2007). While nasal continuous positive airway pressure (CPAP) as an effective form of therapy for OSA has been well documented, the treatment adherence and compliance as measured both subjectively and objectively using downloadable compliance meters remains problematic, despite known cardiovascular, metabolic and quality of life benefits (Grunstein, 2005; Phillips \& Kryger, 2005; Weaver, 2001).

Research has consistently demonstrated the ability of nasal CPAP therapy to improve overall sleep, daytime fatigue, sleepiness, concentration, attention, and mood in OSA (Grunstein, 2005; Weaver, 2001). There is evidence that CPAP treatment reduces hypertension, insulin resistance related to diabetes, inflammatory markers identified in cardiovascular and cerebrovascular disease, and leptin levels associated with obesity in OSA (Won \& Robert, 2000). These studies fail to translate outcomes to weight loss for 
OSA patients, regardless of favorable changes in obesity-related physiologic parameters and laboratory variables in OSA patients treated with CPAP.

While the most common cause of OSA is obesity, the effectiveness of CPAP for weight reduction is not well studied and to date has yielded inconsistent results (Chin et al., 1999; Loube, Loube, \& Erman, 1997; Noseda, Kempenaers, Kerkhofs, Houben, \& Linkowski, 1996; Peppard et al., 2000; Redenius, Murphy, O'Neill, Al-Hamwi, \& Zallek, 2007). Outcome measures of improved sleep architecture, oxygenation, and sleep consolidation have been demonstrated during one or two nights of PSG while using CPAP (Phillips \& Kryger, 2005). Other daytime objective and subjective measures of sleepiness and performance have yielded improvement post-CPAP, yet studies have not addressed the effectiveness of CPAP, nor the extent of improving overall objective measures of continuous sleep/wake activity and energy expenditure. There exists primarily anecdotal and clinical reference to increased energy and activity as a potential benefit of CPAP treatment. Research incorporating the use of portable actigraphy devices could address this research gap by objectively, conveniently, and cost-effectively measuring continuous 24 hour sleep/wake activity and energy expenditure in a patient's

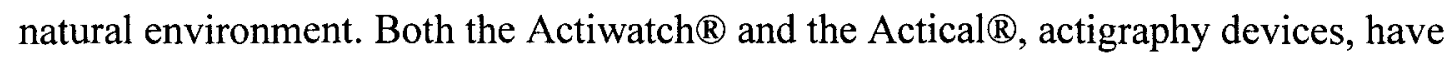
the ability to objectively assess 24-hour sleep and wake activity and energy expenditure, respectively.

Purpose

The purpose of this study was to examine the influence of CPAP therapy on 24hour sleep/wake activity and energy expenditure in overweight and obese OSA adults, as measured by the Actiwatch ${ }^{\circledR}$ and Actical ${ }^{\circledR}$ devices. Specifically, this research measured 
and tested for differences in 24-hour sleep/wake activity and energy expenditure prior and subsequent to initiating CPAP therapy.

\section{Conceptual Framework}

This research study involved physiologic variables and phenomena that were derived from the physical/natural science perspective; however, there was not an existing formal theory, framework, or model that contributed to the structural process diagrammed in the schematic model (Figure 1). Rather, a synthesis of the available literature, combined with over 15 years of clinical sleep experience and observation, guided the framework and provided the theoretical underpinnings. This conceptual framework, was inductively derived, loosely structured with less specific conceptual interrelatedness, not directly tied to explicit theory or theories, and offered a general guidance to the research question and hypotheses.

Variables of significance to this research have been incorporated into the schematic model and include overweight/obesity (BMI), OSA (polysomnography-AHI), sleep activity (actigraphy), wake activity (actigraphy), energy expenditure (actigraphy) and CPAP compliance (internal compliance meter). In this research, the propositions linking these concepts, as currently diagrammed, include the relationships between overweight/obesity and OSA, how sleep fragmentation/disruption and daytime fatigue/sleepiness are associated with OSA, and how (respectively) these concepts link OSA to increased sleep activity and decreased wake activity, with a decrease in 24-hour total energy expenditure. The lower half of the diagram illustrates CPAP treatment, with CPAP compliance affecting sleep consolidation (less sleep fragmentation/disruption), improving wakefulness (less daytime fatigue/sleepiness), and 


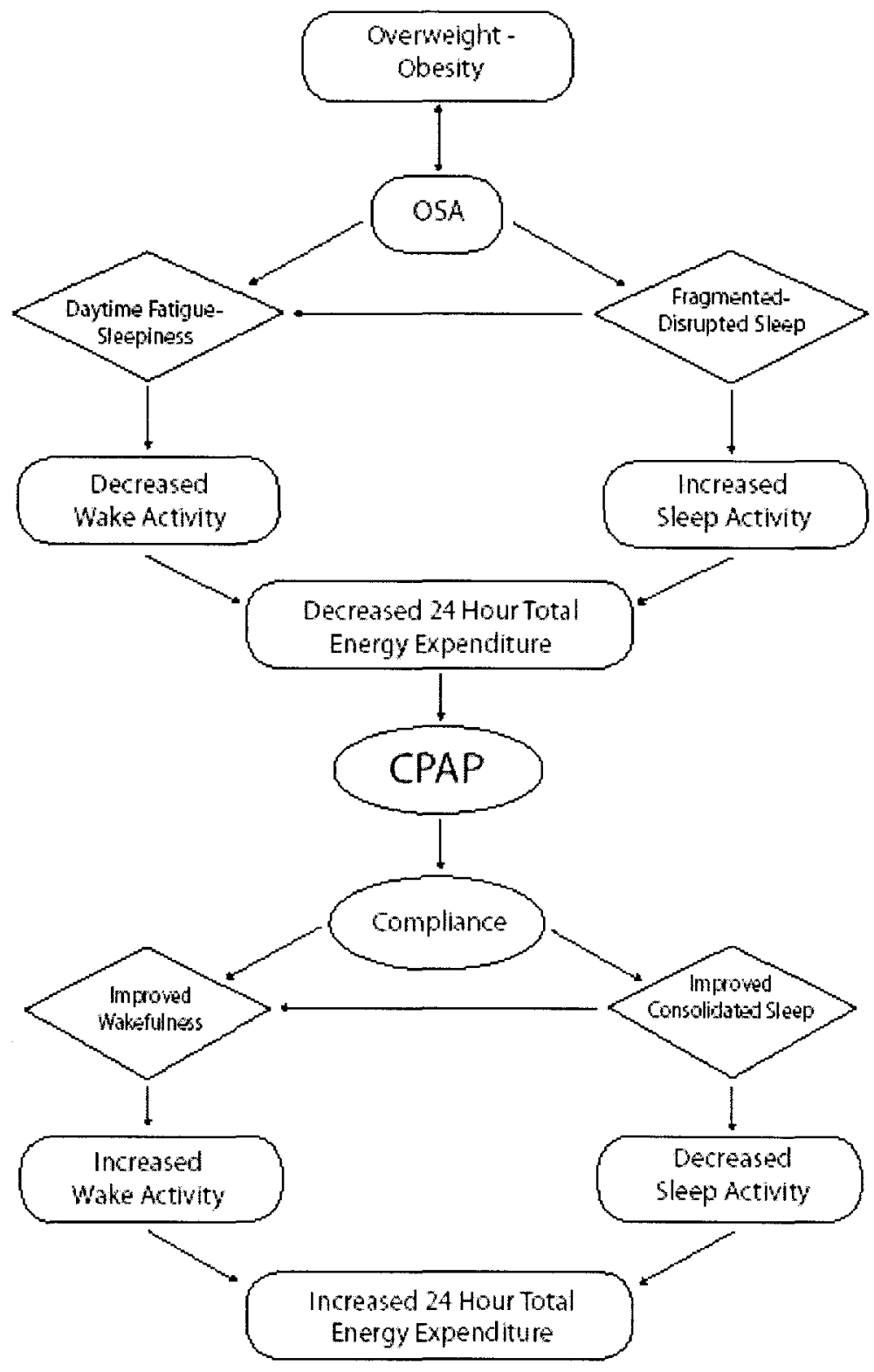

Figure 1. Schematic model illustrating sleep/wake activity and energy expenditure preand post-CPAP in overweight and obese obstructive sleep apnea patients. 
how (respectively) these concepts negatively correlate CPAP treatment (and level of compliance) with sleep activity, and positively correlate CPAP therapy with wake activity and 24-hour total energy expenditure.

\section{Overweight/obesity}

Starting at the top of the Figure 1, overweight and obesity have been defined based on the National Heart Lung and Blood Institute established body mass index (BMI) guidelines for underweight $(<18.5)$, overweight $(25-29.9)$, class 1 obesity $(30-34.9)$, class 2 obesity (35-39.9), and extreme obesity, class 3 (>40) (National Institutes of Health, 1998; National Institutes of Health, National Heart, Lung, and Blood Institute, 2000).

\section{Obstructive sleep apnea}

For this study, OSA has been defined using the diagnostic criteria adopted by the American Academy of Sleep Medicine Task Force (1999), which includes five or more obstructed breathing events per hour of sleep combined with excessive daytime sleepiness (not explained by other factors), or at least two of the following (not explained by other factors): 1) choking or gasping during sleep; 2) recurrent awakenings from sleep; 3) unrefreshing sleep; 4) daytime fatigue/sleepiness; or 5) impaired concentration. A diagnosis of OSA includes clinical symptoms confirmed by polysomnographic findings demonstrating an $\mathrm{AHI} \geq 5$, using the 2007 AASM scoring guidelines (Iber, Ancoli-Israel, Chesson, \& Quan, 2007).

Obesity and obstructive sleep apnea

As diagrammed, the schematic model demonstrates a link from overweight/obesity to obstructive sleep apnea, and is supported by evidence that increased weight and obesity is one of the strongest identifiable risk factors for OSA (Guilleminault \& Bassiri, 
2005). While the link between OSA and obesity has been well recognized, the association is complex and not completely understood (Wolk \& Somers, 2006). Collectively, the cluster of mediating factors related to increased sympathetic activity, inflammation, endothelial dysfunction, oxidative stress, and hypercoaguability are postulated mechanisms contributing to the link between OSA and obesity and associated cardiovascular risk and metabolic dysfunction (Jean-Louis et al., 2008; Miller \& Cappuccio, 2007; Penev, 2000); however, it has yet to be confirmed that obesity is a direct cause rather than just a component OSA. These findings support the bi-directional relationship between overweight/obesity and OSA as diagrammed.

\section{Fragmented/disrupted sleep}

Figure 1 illustrates relationships between OSA and fragmented/disrupted sleep and daytime fatigue/sleepiness, with independent linkages from OSA to fragmented/disrupted sleep and daytime fatigue/sleepiness. An additional link from fragmented/disrupted sleep to daytime fatigue and sleepiness is diagrammed, as well. It is the arousals and awakenings resulting from apneic events that underlie the relationship with fragmented/disrupted sleep, which is frequently subjectively reported by those with OSA. Objectively, a polysomnographic arousal is defined by the AASM (Iber et al., 2007) as at least 3 seconds of EEG shifting from alpha or theta (wake) waveforms followed by 10 seconds of continuous sleep preceding and following the change; whereas, an awakening is at least 30 seconds of alpha or theta (wake) waveforms (Iber et al.).

Daytime fatigue/sleepiness

The most common complaint from those with OSA is daytime fatigue and/or sleepiness (Guilleminault \& Bassiri, 2005; Weaver, 2001; Weaver \& George, 2005). 
These terms are conceptually different; however it is not the concern of this study to objectively operationalize these concepts. These are considered subjective reports most commonly associated with daytime symptoms of OSA and may range from feeling tired or drowsy to napping or falling asleep (Guilleminault \& Bassiri). The relationship between OSA and sleep fragmentation and disruption is believed to result in daytime fatigue and/or sleepiness. It has also been postulated that the direct effects of hypoxemia, carbon dioxide retention, and sympathetic nervous system activation on visceral obesity, insulin resistance, and hypercytokinemia lead to daytime fatigue and sleepiness in OSA patients (Ryan, Taylor, \& McNicholas, 2005; Vgontzas et al., 2000).

\section{Sleep and wake activity}

Sleep disruption and arousal are included in the constellation of pathophysiological manifestations of OSA and are usually objectively measured using polysomnography (American Academy of Sleep Medicine Task Force, 1999). For purposes of this study, activity refers to gross motor (physical) movements as measured using an accelerometer (actigraphy) to distinguish 24-hour activity during wake and sleep. Polysomnographically, sleep activity is measured using electroencephalographic (EEG) measures and scored based on EEG waveforms, rather than measuring physical sleep disturbance or movements (Iber et al.). As measured by actigraphy, increased sleep activity in those diagnosed with OSA is associated with disruption and arousal during nighttime sleep resulting in greater total 24-hour sleep time. In terms of polysomnography, wake activity refers to alpha EEG waveforms consistent with wake. In this study, decreased wake activity in those diagnosed with OSA refers to less physical 
movement and activity during the day with less total 24-hour wake time (measured by actigraphy) versus actual EEG wake activity.

In the upper half of the proposed model, OSA depicts a relationship with decreased wake activity (decreased physical movement with less total 24-hour daytime wake) and increased daytime fatigue and sleepiness. Studies have revealed subjective sleepiness in those with OSA as measured by sleepiness scales (Roehrs, Carskadon, Dement, \& Roth, 2005). Likewise, objective sleepiness has been demonstrated in OSA with controlled maintenance of wakefulness studies (MWT) and multiple sleep latency tests (MSLT) (Carskadon \& Dement, 2005; Roehrs et al.). To date, there have been only a few studies conducted to assess unrestricted daytime physical activity in OSA patients. It seems conceptually reasonable that an individual with OSA who experiences sleep fragmentation and disruption resulting in daytime fatigue and sleepiness would be less physically active during waking hours, even without empirical data. Jean-Louis et al. (2008) and Penev (2007), as well as others (Guilleminault \& Bassiri, 2005; Vgontzas et al. 1994; Weaver \& George, 2005), have at least hypothesized or provided anecdotal reference to these phenomena.

\section{Energy expenditure}

The sum of activity during sleep and wake can be translated to total daily energy expenditure, with weight management a balance of energy intake and energy expenditure. Energy intake is measured by total kilocalories of dietary or nutritional food and beverage consumed, while energy expended is measured by level of activity, inclusive of both sleep and wake. The three main components of energy expenditure include basal metabolic rate (BMR) and resting metabolic rate (RMR), dietary-induced thermogenesis 
(DIT), and physical activity or non-resting energy expenditure (Summerfield, 2001).

While metabolic rate and dietary thermogenesis have been studied in OSA patients for a single 24-hour duration under controlled metabolic chamber conditions (Major, Series, \& Tremblay, 2007; Ryan, Love, \& Buckley, 1995; Stenlof, Grunstein, Hedner, \& Sjostrom, 1996), this research is interested in non-resting energy expenditure or physical movements during sleep and wake, such as fidgeting, random muscle movements, activities of daily living, exercise, and other movements or activities that can be measured using actigraphy, over multiple days and translated to energy expenditure.

Research involving OSA and energy expenditure was limited and offered no clear consensus on whether OSA increases or decreases sleeping EE in OSA patients. Daytime wake time has been even less well-studied, but anecdotally, is thought to be reduced in OSA patients as a result of sleepiness and fatigue (Ancoli-Israel, 2005). Overall, these limited findings do not provide substantive support of energy expenditure changes in OSA, and further highlight the need for research to address this knowledge gap.

\section{Continuous positive airway pressure}

Continuous positive airway pressure (CPAP), when used, leads to less fragmented sleep, improves variable oxygen desaturation and changes, and decreases cardiovascular workload and other forms of autonomic nervous system stimulation resulting in favorable cardiovascular outcomes (Doherty, Kiely, Swan, \& McNicholas, 2005; Heitmann et al., 2004; Kaneko et al., 2003; Pepperell et al., 2002; Stoohs et al., 1993), less cerebrovascular disease and better metabolic profiles (Babu, Herdegen, Fogelfeld, Shott, \& Mazzone, 2005; Becker et al., 2003; Chin et al., 1999; Harsch et al., 2003; Harsch et al., 2004). Furthermore, CPAP has shown improvements in neuropsychologic and 
cognitive dysfunction (Bardwell, Ancoli-Israel, Berry, \& Dimsdale, 2001; Borak et al., 1996; Derderian, Bridenbaugh, \& Rajagopal, 1998), sleepiness (Yamamoto et al., 2000), depression (Means et al., 2003; Schwartz, Kohler, \& Karatinos, 2005), and impaired quality of life (Jenkinson, Stradling, \& Peterson, 1997; Sin, Mayer, Man, Ghahary, \& Pawluk, 2002).

The lower half of Figure 1 links CPAP compliance with less fragmented sleep and improved wakefulness, then links CPAP compliance to decreased 24-hour total sleep activity (measured in minutes via actigraphy) and increased 24-hour total wake activity (measured in minutes via actigraphy). The model terminates with an increase in 24-hour energy expenditure (as measured in kilocalories via actigraphy). As depicted in Figure 1, it is hypothesized that there is an association between CPAP compliance and sleep activity, wake activity and energy expenditure. That is, those who are more compliant with CPAP are more likely to exhibit increased wake activity, decreased sleep activity, and greater energy expenditure.

\section{Actigraphy}

Twenty-four hour energy expenditure, in kilocalories, can be measured using the Actical ${ }^{\circledR}$ actigraphy device. Likewise, 24-hour sleep and wake activity can be measured, in minutes, using the Actiwatch ${ }^{\circledR}$ actigraphy device. Hedner et al. (2004) reported actigraphy to be a novel approach for assessing sleep-wake activity in OSA patients; however, only a few studies have used actigraphy to address changes in total 24-hour sleep/wake activity or non-resting energy expenditure following CPAP treatment. 


\section{Hypotheses}

The purpose of this study was to examine the influence of CPAP on sleep activity, wake activity, and energy expenditure in overweight $(\mathrm{BMI}>25)$ and obese $(\mathrm{BMI}>30)$ OSA (AHI $\geq 5$ ) adults. Sleep activity, wake activity and energy expenditure were measured on a group of overweight and obese individuals prior and subsequent to initiating CPAP therapy, testing for differences that exist before and after therapy over time. The framework developed guided this research and addressed each of the following three hypotheses (hypothesis 1 [H1], hypothesis 2 [H2], hypothesis 3 [H3]):

H1) Subjects will have a significant decrease in 24-hour total sleep activity over time as a result of using CPAP therapy (measured by the Actiwatch $囚$ )

H2) Subjects will have a significant increase in 24-hour total wake activity over time as a result of using CPAP therapy (measured by the Actiwatch ${ }^{\circledR}$ )

H3) Subjects will have a significant increase in 24-hour total energy expenditure over time as a result of using CPAP therapy (measured by the Actical ${ }^{\text {) }}$ )

\section{Summary}

The conceptual framework and corresponding schematic model illustrates a structural process that is grounded in the literature, based upon findings from prior studies, and drawn from clinical experience. While illustrated as a structural process, it is the linking of these concepts, which offered direction to developing the purpose of this research, formulating hypotheses, and guiding proposed data collection methods. The literature review and research findings offered sufficient underpinnings to support the conduct of the proposed study and further suggested additional empirical testing using 
current models that link OSA with obesity, cardiovascular disease and metabolic dysfunction as a framework for larger prospective studies.

The schematic model provided the concepts and framework to guide this research in examining sleep activity, wake activity, and total calculated energy expenditure in overweight and obese OSA patients before and after CPAP treatment using the

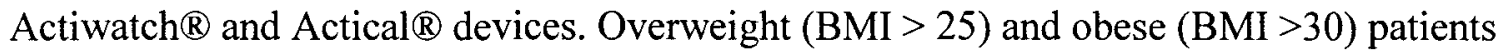
were the targeted population for this study, as their risk of comorbidities are greater than those non-overweight/obese OSA patients. As research has shown, OSA (AHI $\geq 5)$ leads to disrupted and fragmented sleep, which increases energy expended during sleep. This sleep disruption results in daytime fatigue and sleepiness, which may reduce 24-hour total wake time, as determined by physical movement using actigraphy. Because there are two-thirds more hours of wake time than sleep, decreases in total 24-hour energy expenditure (caloric expenditure) may be expected. Following CPAP treatment it would be expected that these OSA patients will experience less sleep disruption and fragmentation, resulting in less total nighttime sleep, lower energy expenditure during sleep, less daytime sleepiness and fatigue with increased wake time activity. As stated in the hypotheses, and following the schematic model, the net loss of energy expenditure during sleep should be well compensated for during waking hours, and result in an increase in overall 24-hour energy expenditure following CPAP therapy. 


\section{CHAPTER II}

\section{LITERATURE REVIEW}

Introduction

This review of the literature will be divided into sections relevant to the concepts and variables of interest to this study. This chapter will provide an initial overview and discussion of sleep, obstructive sleep apnea, continuous positive airway pressure and overweight/obesity. Sleep and wake activity and energy expenditure as the study outcome variables of interest will be included, followed by a separate sleep-related review of actigraphy as the primary measurement instrument for this study. A brief introduction about sleep and the consequences of inadequate sleep and sleep disorders will provide the foundation to support the importance of sleep for maintenance and promotion of physical and mental health. OSA will be discussed as one of the most prevalent sleep disorders and will include a review of OSA pathogenesis, symptoms, diagnosis, health risks and treatment. A separate section will be devoted to CPAP therapy, as the most commonly prescribed treatment for OSA, and will address issues pertaining to compliance and effectiveness. Because OSA is associated with overweight and obesity, this review will explore the current state of the science as it relates to the link between obesity and OSA, associated health consequences and weight loss approaches. Furthermore, as the primary variables of interest to this study, the literature will be examined to identify relationships between sleep activity, wake activity and energy expenditure in OSA. Lastly, actigraphy, 
as the research instrument used to measure sleep/wake activity and energy expenditure will be included in the review. Each of these areas will be explored in depth, highlighting the most relevant research findings as it pertains to the present study of 24-hour sleep activity, wake activity, and energy expenditure in overweight and obese OSA subjects prior to and following CPAP therapy.

\section{Sleep}

Sleep comprises about one-third, or approximately eight hours, of a 24-hour day. The understanding of sleep has historically evolved from simple subjective observations to more objective measures of physiologic and behavioral functions, distinguishing normal sleep from abnormal sleep. Conceptually, sleep has shifted from an intuitive belief of a passive state of brain and body inactivity, somewhere between wakefulness and death, to a more highly organized view of sleep as a complex state which is both qualitatively and quantitatively different from wakefulness, and is initiated and maintained by specific physiologic and behavioral mechanisms (Dement, 2005). Sleep involves a balance between physiology (circadian cyclic activity involving homeostatic regulatory neurologic and hormonal processes) and behavior (sleep-wake schedules, diet and physical activity, along with environmental, societal and cultural influences and demands). Any disruption to either physiologic or behavioral processes may lead to insufficient sleep, sleep disruption and sleep disorders. Individual consequences associated with sleep disorders result from abnormal or inadequate sleep, and may include varying degrees of irritability, moodiness, fatigue, tiredness, sleepiness, somnolence, and lethargy with decreased motivation, attention, memory, and concentration. (Hodgson, 1991; Vaughn \& D'Cruz, 2005). Furthermore, poor sleep and 
sleep disorders have been shown to decrease productivity, reaction time, and attention span. Evidence has also linked poor sleep and sleep disorders to increased health risks such as obesity, hypertension, vascular disease, heart disease, stroke, and diabetes (Vaughn \& D'Cruz). Depression, anxiety and substance abuse have also been associated with inadequate sleep (Lee \& Ward, 2005). More recent studies have linked obesity to inadequate, restricted, or short sleep duration among various age groups and populations (Gangwisch, Malaspina, Boden-Albala, \& Heymsgield, 2005; Hasler et al., 2004; Patel, Malhotra, White, Gottlieb, \& Hu, 2006; Spiegel, Tasali, Penev, \& Van Cauter, 2004; Taheri, Lin, Austin, Young, \& Mignot, 2004;). Additionally, safety issues related to sleep and sleepiness have been more widely recognized and publicized, primarily the risk of motor vehicle, commercial, and industrial accidents (Rosekind, 2006).

Sleep is considered a basic need and as critical to health as nutrition and physical activity (U.S. Department of Health and Human Services [USDHHS], 2003). Even with substantial sleep knowledge, there exists pervasive illiteracy and ignorance as to the importance of sleep and the relationship between sleep, sleepiness, wake function, and performance capacity. This pervasiveness has persisted as a direct result of the failure to adequately translate existing scientific knowledge linking sleep, sleep disorders, and health to broad-based research programs, educational initiatives, clinical practice settings, and public health policy agendas (Institute of Medicine Committee on Sleep Medicine and Research Board on Health Sciences Policy, 2006; Walsh, Dement, \& Dinges, 2005).

\section{Obstructive Sleep Apnea}

It has been estimated that 50 to 70 million Americans suffer with sleep disorders (USDHHS, 2003). Obstructive sleep apnea is just one of more than 70 sleep disorders 
affecting 18 million Americans that disrupt normal sleep architecture and physiology, resulting in many individual and public health risks and consequences (National Sleep Foundation, 2002; USDHHS, 2003). These risks and consequences have generally been poorly translated to public health agendas. More recently, steps have been made toward this effort with the introduction of objectives related to OSA medical evaluation and long-term management, and sleep-related motor vehicle safety into the Healthy People 2010 datasets (Healthy People 2010).

Obstructive sleep apnea (OSA) is considered the most common of the sleeprelated breathing disorders. Early descriptions of OSA date back to the 1800 s, with a well known reference to the Pickwickian Syndrome, named for Joe, an obese, excessively sleepy, snoring boy as described in 1836 by novelist Charles Dickens in the "Posthumous Papers of the Pickwick Club" (Dement, 2005). It was not until 1965 that OSA was medically diagnosed using polysomnography in patients with pickwickian syndrome (Dement), and not until 1970 that OSA was identified in non-obese individuals (Dement). Pickwickian syndrome is now most often referred to as obesity-hypoventilation syndrome which is characterized by obesity, snoring, plethoric complexion, cyanosis, peripheral edema, and right-sided heart failure. While OSA patients may have common features associated with pickwickian syndrome, the obesity-hypoventilation syndrome is classified separately and is considered the most severe form of sleep-disordered breathing (Phillips \& Kryger, 2005, Dempsey, Veasey, Morgan, O'Donnell, 2010). Sleepdisordered breathing generally ranges from severe obesity-hypoventilation syndrome to mild, simple and benign snoring. OSA is the most common of the sleep-related breathing disorders and is also used interchangeably with obstructive sleep apnea-hypopnea 
syndrome (OSAHS). For purposes of this study, OSA encompasses the spectrum of obstructive apneas, hypopneas and respiratory disturbance associated with sleep.

Obstructive sleep apnea is an obstruction in upper airway breathing during sleep. Apneas (and hypopneas) are brief periods of reduced or absent airflow resulting from a complete or partial upper airway collapse while sleeping. From epidemiologic studies based on the general population or community cohorts, the incidence of OSA has been estimated to be between two and four percent of middle-aged men and women, with men affected more frequently than women (Kripke et al., 1997; Young et al., 1993). In more recent years, an exhaustive epidemiologic review of the literature conducted by Young, Peppard, and Gottlieb (2002), found that up to five percent of adults in Western countries are likely to have OSA. While there are numerous studies of infants, children, adolescents and the elderly with OSA, the scope of this study is limited to adults, ages 18 to 65 .

Other factors significant to OSA prevalence rates include menopause, race and ethnicity. Bixler et al. (2001) found that after menopause, the prevalence in women equals that of men. Increased risk and higher prevalence rates of OSA has also been found in African Americans, Latinos and Asians with craniofacial features identified as the primary contributing factor (Ip et al., 2001; Scharf et al., 2004).

\section{Pathogenesis}

The pathogenesis of OSA primarily relates to repeated upper airway collapse as a result of sleep-related neuromuscular compensatory loss and abnormal upper airway anatomy, either caused by adiposity or craniofacial structure (Nguyen, Yim, \& Malhotra, 2007). Structurally, the upper airway consists of the nasopharynx, oropharynx, and hypopharynx. Collapsibility may be favored based on the size of the upper airway lumen, 
inclusive of the tongue, pharyngeal walls, and adipose tissue. Posterior displacement of the mandible and inferior placement of the hyoid bone is often implicated in upper airway collapse (Miles, Weyant, Forrest, \& Rockette, 1996). Clinically, these deformities are referred to as a small chin, overbite, or retrognathia. Schwab et al. (1995) identified the volume of soft tissue structures surrounding the upper airway as an important OSA factor, primarily the lateral oropharyngeal structures such as oropharyngeal muscles, the pharyngeal constrictors, adipose tissue, and lymphoid tissue. Both inflammation and edema of the soft tissues have been described as both a cause and effect of OSA (Miles et al.). Additionally, an oropharynx which has a longer anterior to posterior shape, and a shorter lateral orientation, is more likely to increase predisposition to upper airway narrowing, resistance, and collapse (Malhotra et al., 2002). Nasal obstruction further increases negative pharyngeal pressure, airway resistance and collapsibility, and may be related to deviated nasal septum, mucosal edema and inflammation. Although nasal obstruction is significant, the effect on OSA is variable and not considered primary (Pevernagie, Demeyer, \& Claeys, 2005). Other causes of upper airway collapse include involvement of neuromuscular control, lung volume, ventilatory control instability, $\mathrm{CO} 2$ reserve and loop gain, arousal threshold, and neurotransmitters (Miles et al.); all of which are all relevant, but the complexity of physiological involvement is beyond the scope of this study for inclusion.

Overall, the consensus is that OSA results from predisposing anatomical factors and structural features combined with a decrease in neuromuscular compensation during sleep. While anatomical structure is critical to evaluating OSA, it does not exclusively account for the severity of OSA. The primary risk factors for OSA include obesity with 
android-type fat distribution in the neck and abdomen, crowded and narrow oropharynx, nasal resistance, mandibular deformity and retrognathia, male gender, menopausal women, and certain ethnicities such as African Americans, Latinos and Asians (Ramar \& Guilleminault, 2007). Other risk factors include middle-age (40 to 65 years old), smoking, alcohol and sedative use, snoring, mouth breathing, supine sleep position, and conditions causing macroglossia, stroke or neuromuscular disease, congenital abnormalities affecting craniofacial structure, and polycystic ovarian syndrome (Ramar \& Guilleminault; Young et al., 2002). A positive family history also increases the risk of OSA, with genetic predisposition related to obesity and inherited craniofacial features (Guilleminault \& Bassiri, 2005).

\section{Symptoms}

Clinically, OSA includes both nighttime and daytime symptoms. Nocturnal symptoms tend to be more specific to OSA, whereas, daytime symptoms are often a result of disrupted sleep, regardless of the cause (Guilleminault \& Bassiri, 2005; Dempsey, et al., 2010). The most common nighttime symptoms or observations of an OSA patient include a pattern of loud snoring, alternating with brief pauses in breathing, and followed by gasping or snorting episodes and a brief awakening. A spouse, family member, or bed partner usually provides the greatest insight into this characteristic nighttime pattern associated with OSA, with apneic episodes reported by about $75 \%$ of bed partners (Hoffstein \& Szalai, 1993). Most OSA patients are unaware of these patterns; however, some patients may report varying degrees of restless or disrupted sleep, diaphoresis, dyspnea or choking sensations, esophageal reflux, nocturia, dry mouth, drooling, and bruxism (Guilleminault \& Bassiri). 
Daytime or wake symptoms of OSA primarily include reports of daytime sleepiness or fatigue. Sleepiness may range from mild drowsiness and occasional napping when disengaged and inactive, to more severe episodes of sleep while engaged or actively eating, talking, or driving. Whether sleepiness is mild, moderate, or severe, there is usually underlying insufficient sleep associated with OSA respiratory-related arousals and sleep disturbance. Many may also experience varying degrees of chronic sleep restriction and/or sleep deprivation. In addition to sleepiness, some patients with OSA describe changes in dexterity, concentration, attention, memory, and judgment (Guilleminault \& Bassiri, 2005; Weaver \& George, 2005). Personality and mood changes have been reported, such as aggressiveness, irritability, anxiety, and depression (Guilleminault \& Bassiri). Morning headaches have been described in about half of OSA patients (Paiva, Farinha, Martins, \& Guilleminault, 1997). Decreased libido or impotence has been reported as well (Guilleminault \& Bassiri). OSA has further been associated with cognitive impairment resulting in decreased work and academic performance, increased accidents, and diminished quality of life (Weaver \& George; Gonsalves, Paiva, Ramos, \& Guilleminault, 2004).

Diagnosis

Diagnosis of OSA is based on symptom presentation and confirmed with polysomnography (PSG). PSG is the standard outpatient diagnostic procedure conducted overnight or during a person's usual sleep hours. This testing quantitatively measures physiologic sleep parameters, inclusive of electroencephalogram (sleep/wake stages), electrooculogram (eye movements), submental electromyogram (chin movement), electrocardiogram (heart rate and rhythm), nasal and oral thermocouples/pressure 
transducers (airflow), snore/noise transducers, chest and abdominal bands (respiratory effort), anterior tibialis electromyogram (leg movements), pulse oximetry (oxygen saturation) and video recording and audio monitoring. Computer software programs are utilized for sleep data acquisition and preliminary analysis. Sleep technologists perform patient hook up with the necessary placement of electrodes and sensors, which is followed by computer and biologic calibrations and recording. During data acquisition, the technologists monitor the patient and data closely, both by computer tracings of recorded physiologic activity and video/audio surveillance. The technologist also initiates (per physician protocol and order) and titrates continuous positive airway pressure (CPAP) treatment for those patients diagnosed with sleep apnea or sleep disordered breathing. A single PSG is usually sufficient for diagnosis (American Thoracic Society \& Medical Section of the American Lung Association, 1989), and if OSA is severe enough, both diagnosis and treatment with CPAP can be accomplished in one night/stay (McArdle et al., 2000). A second night/stay for positive airway titration is indicated for those patients with more moderate OSA or for those unable to be successfully titrated during their first night or stay (Wittig et al., 1984). Using Rechtschaffen \& Kales (1968) scale, sleep data can be scored and analyzed by the sleep technologist, and later reviewed and interpreted by the board certified sleep specialist. Although Rechtschaffen \& Kales has been the standard for sleep (EEG) scoring for several decades, a new scoring manual for sleep and sleep-related events has been adopted by the AASM and is required for accredited sleep centers (Iber, Ancoli-Israel, Chesson, \& Quan, 2007). Alternatives to PSG include portable oximetry and ambulatory home monitoring devices, which offer 
advantages over the laboratory setting, however, have limited diagnostic parameters (Chesson, Berry, \& Pack, 2003).

Level of OSA severity has been somewhat ambiguous and is usually a clinical judgment derived from PSG and based on a calculated apnea-hypopnea index (AHI) or respiratory disturbance index (RDI). The $\mathrm{AHI}$ or $\mathrm{RDI}$ is the most commonly used criteria to establish a diagnosis of OSA and quantify the severity, and is determined by the total number of apnea and hypopneas divided by the number of hours asleep (Phillips \& Kryger, 2005). Findings from the Sleep Heart Health Study (SHHS) generated the greatest consensus about defining apneic and hypopneic events (Sharar et al., 2001; Phillips \& Kryger). This longitudinal study included over 6000 middle- and older-aged individuals with cardiovascular disease who underwent in-home portable PSG (Sharar et al.). Sharar et al. defined apneas as an absence up to a $30 \%$ of baseline airflow for greater than 10 seconds, with hypopneas defined as a reduction up to $70 \%$ of baseline airflow for greater than 10 seconds. This definition further required a four percent or more oxygen desaturation associated with the apneas and hypopneas, but did not include sleep disturbance or arousal (Phillips \& Kryger). These definitions have been more widely accepted due to cardiovascular predictability and reproducibility of findings (Phillips \& Kryger) and have been adopted by the AASM and outlined in detail in their new scoring manual (Iber, Ancoli-Israel, Chesson \& Quan, 2007). The specific criteria for diagnosis of OSAHS adopted by the American Academy of Sleep Medicine Task Force (1999) includes five or more obstructed breathing events per hour of sleep combined with excessive daytime sleepiness (not explained by other factors), or at least two of the following (not explained by other factors): 1) choking or gasping during sleep; 2) 
recurrent awakenings from sleep; 3) unrefreshing sleep; 4) daytime fatigue; or 5) impaired concentration. Severity of apnea has also been a debated issue, with mild OSA between five and fifteen obstructed breathing events per hour of sleep ( $\mathrm{AHI} \geq 5$ and $\leq 15$ ), and moderate to severe greater than 15 apneas or hypopneas per hour of sleep (AHI $>15)$ mild sleep apnea (Bixler et al., 2001; Young et al., 1993; Young, Peppard, \& Gottlieb, 2002).

\section{Health risks}

Health risks associated with OSA have been cited as hypertension (Hu et al., 1999; Nieto et al., 2000; Peppard, Young, Palta, \& Skatrud, 2000; Wolk, Shamsuzzaman, \& Somers, 2003), neuropsychologic and cognitive dysfunction (Bedard et al., 1991; Engleman et al., 2000), impotence (Guilleminault \& Bassiri, 2005), motor vehicle crashes (Horstman, Hess, Bassetti, Gugger, \& Mathis, 2000; Young, Blustein, Finn, \& Palta, 1997), cardiovascular disease (Schafer et al. 2002; Sharar et al., 2001), and stroke (Bassetti \& Aldrich, 1999; Bassetti, Aldrich, Chervin, \& Quint, 1996). OSA has been linked endocrine and metabolic disorders, such as type 2 diabetes mellitus (T2DM) associated with glucose intolerance/insulin resistance (Al-Delaimy, Manson, Willett, Stampfer, \& Hu, 2002; Punjabi, Ahmed, Polotsky, Beamer, \& O'Donnell, 2003; Reichmuth, Austin, Skatrud, \& Young, 2005; Vgontas, Bixler, \& Chrousos, 2005) and increased leptin levels associated with obesity (Ip et al., 2001; Spiegel, Tasali, Penev, \& Van Cauter, 2004). Depression (Schroder \& O'Hara, 2005; Shepertycky, Banno, \& Kryger, 2005), decreased quality of life (Baldwin, Griffith, \& Nieto, 2001; Weaver, 2000;), and increased health care costs (Kapur et al., 1999) have been linked to OSA as well. 
Morbidity and mortality has been reported in large observational studies, with several studies demonstrating a significantly greater risk for those with moderate to severe OSA as compared to the general population (Ancoli-Israel et al., 2001; Kripke et al., 1997; Lavie, Lavie, \& Herer, 2005; Young, Peppard, \& Gottlieb, 2002). Three of the most commonly referenced longitudinal studies that have demonstrated an association with OSA and increased cardiovascular morbidities include the Wisconsin Sleep Cohort Study (Peppard, Young, Palta, \& Skatrud), the Nurses' Health Study (Hu et al., 1999), and the Sleep Heart Health Study (SHHS) (Nieto et al., 2000). These studies have all supported the link between systemic hypertension and OSA. Additionally, the incidence of pulmonary hypertension has been estimated between $17 \%$ and $53 \%$ and is most likely to occur in those with underlying heart or lung disease, or in cases of obesityhypoventilation syndrome (Bady, Achkar, Pascal, Orvoen-Frija, \& Laaban , 2000; Niijima et al., 1999). Similarly, the prevalence of coronary artery disease in OSA patients ranges from $14 \%$ to $65 \%$ (Andreas, Schulz, Werner, \& Kreuzer, 1996), with AHI shown to be an independent risk factor and predictor for coronary artery and ischemic heart disease (Peker, Hedner, Kraiczi, \& Loth, 2000; Schafer et al. 1999). As with coronary artery disease, the prevalence rate of heart failure in OSA patients is quite variable, from 12 to 54\% (Javaheri, 2006; Peppard, Young, Palta, \& Skatrud, 2000), and attributed mainly to large negative intrathoracic pressure increases and hypoxemic episodes during apneic events (Won \& Robert, 2007). Lastly, the SHHS also found a two to fourfold increased odds of cardiac arrhythmias (bradyarrhythmias, premature beats, and atrial fibrillation) in patients with an AHI greater than 30 (Mehra et al., 2006). 
Vascular risks associated with OSA have been explained, at least in part, by increased sympathetic nervous activity related to numerous arousals during sleep secondary to obstructed breathing events (Somers \& Javaheri, 2005; Dempsey, et al., 2010). Collectively, the literature does not agree whether OSA is a cause of cerebrovascular disease; however, several studies have found intima-media thickness of the carotid arteries, carotid plaques, and serum inflammatory markers to be increased in OSA patients (Baguet et al., 2005; Minoguchi et al., 2005). Drager et al. (2005) found AHI to be an independent predictor of stroke, and the severity of OSA was associated with increased risk.

Metabolic risks linked to OSA include the complex contribution of both sleep arousals/fragmentation and increased hypoxia/hypercapnia affecting adipocyte-derived inflammatory mediators, the sympathetic nervous system, and cortisol secretion at the hypothalamic-pituitary adrenal axis (Punjabi \& Beamer, 2005). Thus, the causal pathway of metabolic dysfunction and OSA, as reported by Punjabi and Beamer, includes increases in sympathetic activity, along with changes in hypothalamic-pituitary-adrenal function, and a predisposition to a proinflammatory state. Comprehensive reviews of studies involving OSA and metabolic disorders have shown consistent correlations between the severity of sleep-related hypoxemia, sleep duration, and glucose intolerance and insulin resistance, contributing to the risk of T2DM (Punjabi, Ahmed, Polotsky, Beamer, \& O'Donnell, 2003; Vgontas et al., 2000). Likewise, a growing body of evidence has identified apnea-induced dysfunctional leptin and leptin resistance, further affecting glucose intolerance, insulin resistance, T2DM, hypertension, and obesity (Coughlin, Mawdsley, Mugarza, \& Calverley, 2004; Shimzu et al., 2002). 
Sleepiness poses one of the greatest risks to those with OSA, particularly as it relates to motor vehicle accidents. Sassani et al. (2004) conducted a meta-analysis of studies from 1980 to 2003, and when combined with National Safety Council data, findings revealed more than 800,000 drivers were involved in OSA-related motor vehicle accidents in 2000 , costing $\$ 15.9$ billion and 1400 lives. Drivers with OSA have been found have higher motor vehicle accident rates at $12 \%$ to $31 \%$ versus $3 \%$ to $15 \%$ in drivers without OSA (Horstman, Hess, Bassetti, Gugger, \& Mathis, 2000). Commercial long-haul drivers have been estimated to have a higher prevalence of OSA than the general population, $40 \%$ of whom have reported problems with alertness while driving and $20 \%$ of whom have reported dozing at the wheel (Hakkanen \& Summala, 2000).

\section{Treatment}

To date, continuous positive airway pressure (CPAP) is considered the most uniformly effective treatment for OSA, and is the only intervention shown to consistently improve neurobehavioral and cardiovascular morbidities (Veasey et al. 2006). Unfortunately, as reported by Zozula \& Rosen (2001), CPAP is often refused or poorly tolerated in as many as $25 \%$ to $50 \%$ of OSA patients. In many cases, conservative treatment for OSA is offered first line or combined with CPAP therapy. Conservative treatment focuses on lifestyle and behavioral modifications to improve overall sleep hygiene and sleep apnea. Such measures include maintaining routine sleep/wake activity with eight consecutive hours allocated to sleep, altering the sleep environment to control for comfort, noise and temperature, elevating the head of the bed, lateral or side-lying sleep position, relieving nasal rhinitis and congestion with intranasal corticosteriods, smoking cessation, reducing caffeine intake, avoiding alcohol, relaxants and sedative 
medications, increasing physical activity, and avoiding naps (Phillips \& Kryger). Unfortunately, according to Shneerson and Wright (2007) the value of these first line measures has not been established.

Clinically, weight loss is often a primary recommendation in overweight and obese patients, and has been shown effective in reducing apneic events, particularly in cases where weight gain coincided with worsening of apnea and related symptoms (Phillips \& Kryger, 2005). Despite effectiveness in alleviating OSA, weight loss for overweight and obese patients with moderate to severe OSA usually offers variable success, both short- and long-term (Shneerson \& Wright, 2007). Exercise is often recommended as a weight loss strategy in addition to reduced calorie diets; although, little data are available as to the long-term success of such recommendations (Veasey et al., 2006). An investigational study conducted by Winslow, Bowden, DiDonato, McCullough \& Day (2010) showed promising results for pharmacologically treating OSA when combined with lifestyle modification. This study showed statistically significant weight loss and meaningful improvements in apnea, respiratory-related arousals, nighttime oxygen levels, subjective sleep quality, and blood pressure (Winslow, et al.).

Oral or dental appliances have been used with varying degrees of success in patients who snore, have mild sleep apnea, or are CPAP intolerant. These appliances are consistently preferred over CPAP, and have been shown to be more effective than surgical uvulopalatopharyngoplasty, both short- and long-term (Walker-Engstrom, Tegelberg, Wilhelmsson, \& Ringqvist, 2002). Other surgical intervention for OSA has included tracheostomy, laser-assisted uvulopalatopharyngoplasty (LAUP), septoplasty, 
oromaxillofacial surgery, and radiofrequency volumetric tissue reduction (somnoplasty) (Phillips \& Kryger). Additionally, bariatric surgery has been applied in morbidly obese patients with OSA and has been successful in the treatment of OSA caused by obesity (Buchwald et al., 2004). While surgical options are important treatment considerations for OSA patients, it is beyond the scope of reviewed literature for this study. Generally, as with most forms of OSA treatment, long-term treatment effect and evaluation is insufficient (Phillips \& Kryger). Clearly, studies involving OSA and treatment follow-up would contribute to identifying better outcome measures for such an at-risk population.

\section{Continuous Positive Airway Pressure}

CPAP is considered the primary therapy and treatment of choice for moderate to severe OSA. CPAP was first described in 1981 (Sullivan et al., 1981); although, clinical acceptance and application was not widespread until about 1985 (Grunstein, 2005). CPAP is a continuous positive flow of air pressure delivered via nasal mask, titrated and adjusted to a therapeutic level, and measured in centimeters of water pressure ranging from 5 to $15 \mathrm{~cm} \mathrm{H}_{2} \mathrm{O}$. The nasal mask is secured by headgear and is connected by a 6 foot flexible hose to a lightweight, portable CPAP machine. The CPAP mask is worn during sleep in order to alleviate snoring and apneic events. While CPAP does not increase upper airway muscle activity (Strohl \& Redline, 1986), endoscopic and upper airway imaging has confirmed that CPAP does act as an upper airway pneumatic splint, increasing volume and airway area, reducing pharyngeal wall thickness and edema (Grunstein). The positive pressure is fixed and delivered continuously throughout inspiratory and expiratory cycles. Bilevel positive airway pressure therapy is an alternative to CPAP and is used in select cases to offer independent adjustment of 
inspiratory and expiratory pressures, either to accommodate poor response to CPAP or to improve pressure tolerance and comfort. Ayas et al. (2004) conducted a meta-analysis and found the use of auto-titrating positive pressure machines, versus traditional supervised PSG titrated and fixed continuous pressure machines (CPAP), has become more widely used, but studies have shown no improvement in tolerance or compliance even with modest decreases in pressure. Likewise, a Cochrane review of interventions to improve use of CPAP was conducted and revealed no clear advantage, or supportive data, to warrant replacing traditional, less expensive CPAP for more expensive auto-titrating units in the home (Haniffa, Lasserson, \& Smith, 2004).

\section{Compliance}

Compliance with CPAP has been referred to in terms of acceptance, prescription, adherence, tolerance and usage (Grunstein, 2005). The most widely used term is compliance and will be the preferred term used in this study to indicate objective measurement of total time wearing CPAP while delivering the established and prescribed pressure. In recent years, CPAP machines have been manufactured and equipped with a sophisticated meter to measure actual compliance using a removable card and computer software to download compliance data (Grunstein). CPAP compliance studies have demonstrated wide ranges in findings. Some report $58 \%$ to $80 \%$ of patients refuse CPAP after initial trial, while others report a 50\% CPAP purchase rate. Objective monitoring of CPAP compliance has revealed about $46 \%$ of OSA patients used CPAP 4 hours or more on at least $70 \%$ of the observed nights, with compliance at one month predictive of compliance at 3 months (Kribbs et al., 1993; Weaver et al., 1997). For lack of other data, this has been cited as the threshold, or minimally accepted value, for compliance. Those 
who do not report symptomatic improvement or are unable to use CPAP at the minimal criteria are considered unresponsive or noncompliant with CPAP therapy.

The major factors influencing compliance are most frequently associated with side effects, noise, aesthetics, bed partner intolerance, and inconvenience. Side effects of CPAP usually lead to poor compliance or discontinued use, and include rhinorrhea, nasal congestion, nasal/oropharyngeal dryness, epistaxis, skin abrasion/rash, conjunctivitis related to air leak, chest discomfort, sinus pressure, claustrophobia, and difficulty exhaling (Grunstein, 2005). There are multiple mask and interface options available to maximize comfort and fit for patients. Likewise, a ramp feature to allow gradual pressure increase is available on most CPAP machines, as is a built in humidifier to relieve dryness. Despite improvements in the size, shape, weight, and noise of CPAP machines, as well as mask interfaces, humidification and various accessories, compliance remains an issue. Hoy et al. (1999) demonstrated that education and positive reinforcement by the healthcare provider improves compliance; however, the degree to which is necessary is not clear. Results of a meta-analysis confirmed that patients who have more severe symptoms and are sleepier at baseline have greater objective CPAP usage (Patel et al., 2003).

\section{Health benefits}

CPAP, when used, leads to less fragmented sleep, improves variable oxygen desaturation and changes and decreases cardiovascular workload and other forms of autonomic nervous system stimulation. CPAP has also been shown to improve leptin and ghrelin levels, central obesity, and insulin sensitivity (Babu, Herdegen, Fogelfeld, Shott, \& Mazzone, 2005; Chin et al., 1999; Harsch et al., 2003; Harsch et al., 2004). 
Furthermore, findings suggest CPAP can reverse or reduce the risks associated with OSA, including mortality (Lavie, Lavie, \& Herer, 2005; Marti et al., 2002), hypertension (Becker et al., 2003; Heitmann et al., 2004; Pepperell et al., 2002;), cardiovascular disease (Doherty, Kiely, Swan, \& McNicholas, 2005; Kaneko et al., 2003; Stoohs et al., 1993), cerebrovascular disease (Martinez-Garcia et al., 2009), neuropsychologic and cognitive dysfunction (Bardwell, Ancoli-Israel, Berry, \& Dimsdale, 2001; Borak et al., 1996; Derderian, Bridenbaugh, \& Rajagopal, 1998), sleepiness (Yamamoto et al., 2000), depression (Means et al., 2003; Schwartz, Kohler, \& Karatinos, 2005), impaired quality of life (Jenkinson, Stradling, \& Peterson, 1997; Sin, Mayer, Man, Ghahary, \& Pawluk, 2002), impotence (Karacan \& Karatas, 1995), car crashes, and health care costs (Findley $\&$ Suratt, 2001; Sassani et al., 2004). It has been estimated that treating all drivers who have OSA with CPAP would save 900 lives and yield a net savings of $\$ 11$ million per year (Sassani et al.).

Although the benefits of nasal CPAP as an effective form of therapy for OSA has been well documented, treatment adherence and compliance remains problematic, despite known cardiovascular, metabolic, and quality of life benefits. While the data suggests favorable effects of CPAP, many questions and gaps remain, which include compliance, responsiveness to therapy, and long-term benefits (Caples, Gami, \& Somers, 2005). According to Grunstein (2005) and Basner (2007), true CPAP compliance remains unknown, as most follow-up studies have biases related to selected patient populations and sampling. To address this need, the current and ongoing multi-center National Heart Lung and Blood Institute (NHLBI)-funded Apnea Positive Pressure Long-term Efficacy Study (APPLES) has been developed and designed to evaluate the long-term 
effectiveness and benefits of CPAP in relation to improving neurocognitive function, mood, sleepiness, and quality of life (Kushida, 2007). Likewise, efforts from the Centers for Disease Control and Healthy People 2010 epidemiologic and surveillance studies may provide future insight into follow-up, long-term management and outcomes of CPAP therapy in patients with OSA. Additional research addressing responsiveness to therapy in terms of sleep and wake activity and CPAP compliance would offer contribution toward filling the gaps in the current body of knowledge and state of sleep science.

\section{Overweight and Obesity}

Obesity is one of the strongest identifiable risk factors for OSA. It has been estimated that $40 \%$ of all obese patients have OSA, and $70 \%$ of all patients with OSA are obese, with the risk of OSA in obese individuals ten times greater than that of the general population (Vgontzas et al. 1994). Although not completely understood whether a cause or effect, cross-sectional studies have endorsed the relationship between obesity and OSA to be primarily associated with increased body mass index, central obesity, upper airway fat distribution, and large neck size (Tasali \& Van Cauter, 2002; Tung, 2005; Young, Peppard, \& Gottlieb, 2002).

Overweight is considered weight in excess of the average for a given height; whereas, obesity is defined as an excess of body fat (Stunkard \& Wadden, 1993). Obesity can be classified by level of fatness (total fat percentage of body weight), distribution of fat (location of stored fat), and body weight (Summerfield, 2001). The most commonly used measure to classify body weight is body mass index (BMI). BMI can be calculated using the formula: weight in kilograms divided by height in meters squared. The NHLBI established guidelines based on BMI for underweight ( $<18.5)$, overweight (25-29.9), 
class 1 obesity (30-34.9), class 2 obesity (35-39.9), and extreme obesity, class 3 (>40) (National Institutes of Health, 1998; National Institutes of Health, 2000).

Obesity trends from the U.S. Centers for Disease Control and Prevention (CDC), Behavioral Risk Factor Surveillance System (2007) graphically demonstrate dramatic increases in the prevalence of obesity (BMI $\geq 30)$ in the United States from 1985 to 2006 (U.S. Department of Health and Human Services \& Centers for Disease Control and Prevention, 2007). Of the states participating in the BRFSS in 1990, 10 had a prevalence of obesity less than $10 \%$ and no states had prevalence equal to or greater than $15 \%$ (U.S. Department of Health and Human Services \& Centers for Disease Control and Prevention). By 1998, no state had prevalence less than $10 \%$, seven states had a prevalence of obesity between 20 and $24 \%$, and no state had prevalence equal to or greater than $25 \%$ (U.S. Department of Health and Human Services \& Centers for Disease Control and Prevention). In 2006, only four states had a prevalence of obesity less than $20 \%, 22$ states had a prevalence rate equal to or greater than $25 \%$, and 2 states (Mississippi and West Virginia) had equal to or greater than 30\% (U.S. Department of Health and Human Services \& Centers for Disease Control and Prevention). These alarming figures have continued to rise, and even more recent data collected from 2009 has shown only one state (Colorado) and the District of Columbia to have had a prevalence of obesity less than $20 \%$. (U.S. Department of Health and Human Services, Centers for Disease Control and Prevention, 2011). According to National Health and Nutrition Examination survey (NHANES) data from 2003-2004, 66.3\% of adults age 20 and older are overweight or obese, and 32\% are obese (National Center for Health Statistics, 2007). This same data reveals the prevalence of overweight in adolescents age 
12-19 to be $17 \%$, and children age 6-11 is 19\% (National Center for Health Statistics). While the adult population is of interest to this study, overweight and obesity in children and adolescents have current, as well as future adult health implications. Overweight and obesity poses a major public impact in terms of health, healthcare costs, and quality of life.

Specific factors implicated in the obesity epidemic include activity level and physical fitness, diet and nutritional intake, drug and alcohol use, and smoking (Summerfield, 2001). Health risk factors are associated with the level of overweight or obesity (higher BMI), age of onset and duration (younger and longer), location of fat storage (central or visceral), and race-ethnicity (African American and Hispanic) (National Institutes Of Health, 1998). Obesity is directly linked to cardiovascular disease and hypertension, (Wolk, Shamsuzzaman, \& Somers, 2003; Wolk \& Somers, 2006). In addition, dyslipidemia, T2DM, cancer, osteoarthritis, pregnancy complications and sleep apnea are associated with obesity (Caterson et al, .2004; Flegal, Graubard, Williamson, \& Gail, 2005; Summerfield, 2001; Wilson, D'Agostino, Sullivan, Parise, \& Kannel, 2002). As previously discussed, findings have shown OSA to be not only a health risk factor associated with obesity, but an independent contributing factor to many of these same comorbidities (Caterson et al, .2004; Dyken, Ali, Raman, \& Eppen, 2007; Mullis et al., 2004). Furthermore, there is supportive evidence that metabolic and endocrine changes which promote obesity and diabetes may be a result of not only sleep disruption and arousal caused by OSA, but sleep loss and overall short sleep duration, as well (Chaput et al. 2007; Hasler et al., 2004; Spiegel, Leproult, \& Van Cauter, 1999; Taheri, Lin, Austin, Young, \& Mignot, 2004). 
Obesity and obstructive sleep apnea

Many studies have identified the relationship between obesity and OSA, with cross-sectional studies demonstrating increased body weight parallels the risk and severity of OSA (Young, Peppard, \& Gottlieb, 2002). Prospective population-based studies have shown that a $10 \%$ weight gain resulted in a sixfold increase in developing OSA, while a $10 \%$ weight loss decreased the AHI by $26 \%$ (Peppard, Young, Palta, \& Skatrud, 2000). As reported by Young et al. (2002), the Wisconsin Sleep Cohort study, and the Cleveland Family Study revealed findings consistent with excess body weight and central obesity to be a significant predictor of OSA and increased AHI . Specifically, a BMI of $>28 \mathrm{~kg} / \mathrm{m}^{2}$ has been shown to increase the risk of OSA in both middle-aged men and women (Ancoli-Israel et al., 2001). Although the prevalence of OSA associated with central obesity continues to increase with age (Kripke et al., 1997), the link between obesity and OSA has been shown to decline for both genders after age 50 (Ancoli-Israel et al.; Young et al; Young, Peppard, \& Gottlieb).

\section{Weight loss approaches}

When considering weight loss approaches as treatment for obese and overweight OSA subjects, CPAP has been shown to decrease total body fat and central adiposity in only a few studies (Chin et al., 1999; Loube, Loube, \& Erman, 1997; Noseda, Kempenaers, Kerkhofs, Houben, \& Linkowski, 1996). Interestingly, Peppard, Young, Palta, and Skatrud (2000) found newly diagnosed patients with OSA have a greater increase in weight the year prior to diagnosis, and patients who comply with CPAP tended to gain weight. Redenius, Murphy, O'Neill, Al-Hamwi, and Zallek (2007) also noted increased BMI in women after 1 year of CPAP, with no BMI change in controls. 
Buchwald et al. (2004) conducted a systematic review and meta-analysis of bariatric surgery and indicated the impact of surgical weight loss, particularly gastric bypass, on OSA and AHI demonstrated favorable results. Grunstein, Stenlof, Hedner, Peltonen, Karason, and Sjostrom (2007) found improvement in the incidence of sleep apnea symptoms, diabetes, and hypertriglyceridemia two years following bariatric surgery. A study examining three non-surgical treatments for OSA (CPAP, oral appliance, and weight loss) found CPAP resulted in the most improvement in physiologic, symptomatic and quality of life measures, followed by oral appliance (Lam et al., 2007). In this study, weight loss demonstrated improved sleep parameters, but weight control was not uniformly effective (Lam et al.). Kajaste, Brander, Telakivi, Patinen, and Mustajoki (2004) evaluated a cognitive-behavioral weight reduction program in the treatment of OSA, both with and without CPAP, and found weight loss was achieved with this program; however, adding CPAP did not offer any greater weight loss. Despite routine recommendations about the importance of weight loss in the treatment of OSA, there is very little documented clinical intervention. As reported by Peppard et al., the Wisconsin Sleep Cohort Study found that further increases in weight and AHI would result if therapy directed toward overweight and obesity was neglected.

\section{Health consequences}

Obesity has been cited as responsible for between 300,000 and 400,000 deaths per year in the United States (Mokdad et al., 2004; USDHHS, 2001; Allison et al., 1999). Most of these deaths are attributable to coronary artery disease, hypertension, and T2DM (Mokdad et al.; Allison et al., 1999). It has been suggested that this number may be, at least in part, due to the prevalence of OSA in $30 \%$ of adults with a BMI $>30 \mathrm{~kg} / \mathrm{m}^{2}$ and 
$50 \%$ of adults with a BMI $>40 \mathrm{~kg} / \mathrm{m}^{2}$ (Resta et al., 2001). Both direct and indirect costs of obesity have been estimated at $\$ 117$ billion per year related to medical and healthcare costs and associated comorbidities (USDHHS, 2001; Wolf \& Colditz, 1998; Wolf, 1998).

Given the present obesity trend and the strong relationship between obesity and OSA, health and healthcare costs can only be expected to rise exponentially without prioritizing public and individual healthy weight management and weight loss interventions at the national, state and local levels (See, Mensah, \& Olopade, 2006). Programs and initiatives focusing on nutrition and physical activity have been developed with federal, state and local funding; however, sleep, as an important one-third of life and health, has been absent from these initiatives. Research addressing the OSA population, $70 \%$ of whom are estimated to be overweight or obese, has relevance and importance to improving both individual and public health outcomes.

Sleep Activity, Wake Activity, and Energy Expenditure

The sum of activity during sleep and wake can be translated to total daily energy expenditure, with weight management a balance of energy intake and energy expenditure. Energy intake is measured by total kilocalories of dietary or nutritional food and beverage consumed, while energy expended is measured by level of activity, inclusive of both sleep and wake. A kilocalorie $(\mathrm{kcal})$ is determined by the amount of heat required to raise the temperature of 1 kilogram of water 1 degree Celcius, which is measured using a bomb calorimeter.

The 3 main components of energy expenditure include basal metabolic rate (BMR) and resting metabolic rate (RMR), dietary-induced thermogenesis (DIT), and physical activity or non-resting energy expenditure (Summerfield, 2001). BMR is usually 
slightly lower than RMR, which can be more practically measured any time of the day and under less stringent conditions. Simplified, BMR is the total oxygen consumption of the active tissues in the body. Metabolic rate is the calorie expenditure required to support the body's cellular activity. As with measuring kcals, metabolic rates are determined by using direct calorimetry or indirect calorimetry (Summerfield). Direct calorimetry involves the use of a controlled chamber with walls lined with water to measure heat production and energy expenditure by temperature changes in water lining the walls of the chamber. While this method is more accurate, indirect calorimetry (spirometry) is often more practical, but still involves a face mask, hood or mouthpiece to measure inhaled oxygen and produced carbon dioxide using the respiratory quotient for calorie expenditure and estimated energy expenditure (Henning, Lofgren, \& Sjostrom, 1996). RMR is affected by many factors, including body size, thyroid hormone, nutritional status, gender, age, race, and heredity (Summerfield, 2001). Physical activity involves energy expenditure, but changes in RMR are debated by researchers. The type, intensity, and duration of physical activity, as well as age, gender and type and quantity of food ingested following the activity may all have some varied level of influence on RMR. Another factor related to energy expenditure is diet-induce thermogenesis (DIT), which is the production of heat by the body in response to eating, contributing to a rise in RMR. DIT is otherwise known as the thermic effect of food and involves the energy expended associated with dietary ingestion, digestion, absorption, and transport of nutrients, as well as increased sympathetic activity and heat production by brown adipose tissue (BAT) resulting from ingested calories. Lastly, non-resting energy expenditure or activities such as fidgeting, random muscle movements, activities of daily living, exercise 
and other physical activities are included as the final component to measuring energy expenditure.

A more practical measurement of energy expenditure for activity includes doublelabeled water method and heart rate monitoring (Ravussin \& Rising, 1992; Summerfield). The first of which estimates carbon dioxide production from isotopes eliminated in the urine (Ravussin \& Rising), with the latter using heart rate to estimate oxygen consumption and the amount of kcals expended. Metabolic equivalent tasks (MET) have been developed to estimate calorie cost of various physical activities and energy expenditure (Ainsworth et al., 1993). Regardless of how measured, it is ultimately the difference between energy in and energy out that leads to weight gain, loss, and maintenance.

Overweight and obese individuals have higher absolute RMRs, potentially dysfunctional BAT, and are less physically active than those with ideal body weight. As it relates to OSA, it has been shown that energy expenditure in OSA patients is greater during sleep than those without OSA (Bonnet, Berry, \& Arandel, 1991; Stenlof, Grunstein, Hedner, \& Sjostrom, 1996). This is speculated to be due to airway obstruction and hypoxemia/hypercarbia resulting in increased arousals and disruption during sleep, along with increased sympathetic nervous system activity. Waking activity is less well studied, but also speculated to have the potential to be reduced in OSA patients due to daytime sleepiness and fatigue associated with OSA (Ancoli-Israel, 2005). Several studies have evaluated weight gain in OSA patients prior to and following diagnosis, and have evaluated weight loss interventions with and without various treatment modalities (CPAP, low calorie diets, behavioral and surgical intervention) (Buchwald et al., 2004; 
Kajaste et al., 2004; Lam et al., 2007; Peppard et al., 2000; Redenius et al., 2007; Young et al., 2002). These studies confirm the relationship between weight gain, overweight, and obesity and the development of OSA. They also endorse that weight loss, regardless of the intervention, decreases the severity of apneas and hypopneas and can improve OSA symptoms. Albeit limited, there is conflicting evidence regarding the ability of CPAP to reduce weight in OSA patients, with some evidence of weight gain.

Two studies were found that addressed energy expenditure in OSA patients. A study by Ryan, Love, and Buckley (1995) identified a lack of evidence regarding the effects of sleep disorders on energy expenditure. Their research was interested in determining whether resting energy expenditure (REE) during wake and dietary thermogenesis were reduced in OSA patients. Energy expenditure was measured using indirect calorimetry in a metabolic cart and canopy system, further expressed in metabolic body size (Ryan et al.). Patients with OSA had increased REE compared to matched-weight controls; however, once correction was made for lean body mass, both REE and dietary thermogenesis failed to show a difference between groups (Ryan et al.). Measurements were repeated following CPAP therapy in the OSA patients, but again, did not reveal any differences in resting energy expenditure or dietary thermogenesis. Obesity in OSA patients was found to be lifestyle and diet-induced, and related to reduced waking energy expenditure, rather than hypothalamic mechanisms (Ryan et al.). This would be consistent with speculation that OSA patients are more likely to gain weight prior to diagnoses and fail to lose weight following CPAP treatment, despite improvement in the hypothalamic-pituitary adrenal axis (Ancoli-Israel, 2005; Traviss, Barr, Fleming, \& Ryan, 2002). 
Stenlof, Gruenstein and Hedner (1996) examined 24-hour energy expenditure (EE) in 5 male patients with OSA and 6 control snoring subjects. Three months following CPAP treatment in the OSA patients, 24-hour EE was again conducted. In order to measure EE, patients were studied using indirect calorimetry in a controlled metabolic chamber equipped like a hotel room. Each patient was studied for a single 24-hour period, with those using CPAP studied an additional 24-hour period. OSA patients had higher sleep EE and 24-hour EE than the snorers. It was concluded that OSA increases sleep EE higher than quiet wakefulness, and treatment with CPAP decreases sleep EE by an average of $16 \%$ (Stenlof et al.). The researchers explained 3 possible mechanisms which could account for increased sleeping EE in OSA patients. These explanations included increased frequency of arousals during sleep due to apneic events, increased breathing efforts against upper airway occlusion, and increased sympathetic activity of the sympathetic nervous system (Stenlof et al.). There were no observed changes in daytime EE; although the authors indicated the study was limited due to the controlled environment and restriction of patient wake activity required for indirect calorimetry (Stenlof et al.). Stenlof et al. stated that ambulatory measures of EE in OSA patients have yet to be performed, and findings of increased sleep EE in OSA patients have implications for energy and weight management. They further suggested that additional study of OSA patients should be conducted in an unrestricted and natural environment, which may offer utility in the development of weight loss strategies in this population (Stenlof et al.). Despite clinically relevant findings, both studies by Stenlof et al. and Ryan et al. were limited in the number of patients as well as the ability to study these patients in a 24-hour uncontrolled and natural setting. 


\section{Actigraphy}

When evaluating sleep and wake activity, actigraphs, actimeters, accelerometers, or activity monitors have been utilized as devices to record 24-hour movement or activity. For this study, actigraphy is the preferred term used to encompass all forms of this device in this review. In sleep, actigraphs have been most extensively used in the study of circadian rhythms, and were first used to differentiate sleep from wake in the early 1970's (Ancoli-Israel, 2005). With advances in technology, actigraphy has been applied to the evaluation of insomnia, circadian rhythm disorders, sleep-related breathing disorders, response to therapy, and sleep patterns in special populations (Morgenthaler et al., 2007). In these instances, actigraphy has offered objective measurement of sleep duration and circadian patterns of sleep. Actigraphy, however, does not replace electroencephalogram-defined sleep or standard PSG; nor does it account for subjective measures of sleep experiences (Morgenthaler et al.). The device is simply meant to measure movement of a limb and is usually worn on the wrist or ankle. Despite the simplicity, actigraphy is highly sensitive and involves complex and sophisticated algorithms for estimating sleep and wake activity (Ancoli-Israel). The simple basis of this device is that it detects decreased movement or inactivity when sleeping and increased movement or activity while awake. The complex algorithms for scoring data have varying degrees of accuracy depending on the specific device, with some offering greater ability to distinguish wake, sleep, sleep onset, awakenings during sleep and total sleep time (Ancoli-Israel). A number of computer software programs are available with actigraphy devices and offer packages for editing and scoring of activity and sleep/wake data; however, most are used in combination with a manual activity or actigraph log to 
aid in editing specific to time in bed, out of bed, unusual activity during the day or device removal (Ancoli-Israel).

Actigraphy has been reported to be best at estimating total sleep time, but becomes less accurate with disturbed sleep (Elbaz, Roue, Lofaso, \& Quera, 2002). Additionally, several studies have revealed actigraphy may underestimate wake (Hedner et al. 2004; Lichenstein et al., 2006), and a recent epidemiologic study also has reported the potential for overestimating sleep (Lauderdale et al., 2006). Regardless, most studies have consistently shown actigraphy to be a valid assessment of sleep patterns with significant correlation to PSG in measuring total sleep time (Matsumoto et al. 1998; Hedner et al.; Vallieres \& Morin, 2003). Advantages include its cost-effectiveness and ability to record sleep and wake activity in a natural environment, during both night and day for continuous 24 hour and extended periods of time (Ancoli-Israel).

In 2007, the American Academy of Sleep Medicine conducted an evidence-based review of the use of actigraphy in the clinical assessment and management of sleep and sleep disorders. In this review, actigraphy was used to describe a method of measuring increased wake time after sleep onset and "sleep pattern", defined as "the circadian pattern of sleep and wakefulness over multiple sleep cycles" (Morgenthaler et al., 2007, p. 521). Recommendations for the use of actigraphy in clinical practice involved the following 3 areas: 1) actigraphy in the evaluation of sleep disorders; 2) actigraphy in assessing the response to therapy of sleep disorders; and 3) actigraphy in special populations and special situations (Morgenthaler et al.). Practice parameters were developed based on evidence collected from 108 studies reviewed. Approximately 38 of these studies did not compare actigraphy to a reference standard, while 70 of the studies 
compared actigraphy to a standard, such as PSG, phase markers, sleep logs/diaries or subjective estimates of sleep quality (Morgenthaler et al.). Studies have been conducted using actigraphy to determine total sleep time of healthy patients, patients with OSA, and infants, as compared to PSG, and found that there was a $90 \%, 84 \%$ and $84 \%$ agreement in the two measures, respectively (Hedner et al. 2004). Studies have also used actigraphy in instances where PSG was not available to estimate total sleep time in OSA patients, in addition to using combined monitoring of respiratory events and actigraphy to assess the severity of OSA. Lastly, actigraphy has been shown to be useful in determining outcomes and treatment response for circadian rhythm disorders (jet lag and shift work) and insomnia (Morgenthaler et al.).

While both OSA and treatment response were addressed using actigraphy, there were only a few studies published that utilized actigraphy to evaluate response to CPAP treatment in OSA patients (Gadnoadoux, Nguyen, Rakotonanahary, Vidal, \& Fleury, 2004; Albert-Tulkens et al., 1987). Gadnoadoux et al. were interested in correlating total sleep time (TST) in OSA patients using CPAP as measured by actigraphy, as compared to PSG, and found highly correlated sleep times with the exception of overestimations and underestimations in 3 out of 26 patients. Albert-Tulkens et al. used wrist actigraphy to measure a movement and fragmentation index during sleep, and demonstrated higher indices of movement and fragmentation in patients with sleep apnea, with decreased indices following treatment. Neither of these studies (Gadnoadoux et al; Albert-Tulkens) addressed changes in total 24-hour sleep/wake activity after CPAP treatment. More recent publications by West, Kohler, Nicoll, \& Stradling (2009) and Otake et al. (2010) studied the effect of CPAP on sleep/wake activity using actigraphy. West et al. studied 
36 men with OSA and found there were no significant differences in activity levels one week before the initiation of CPAP as compared to one week after CPAP therapy. Otake et al. confirmed agreement between PSG and actigraphy sleep/wake data, and further determined subjects treated after one month of CPAP had significantly less activity levels/movement than prior to CPAP. No additional research was found to support or negate the findings from these studies. In fact, more clinical and anecdotal evidence demonstrates otherwise.

It has been concluded that additional research using actigraphy should be considered to further expand the value in clinical settings (Morgenthaler et al., 2007; Ancoli-Israel, 2005; West et al., 2009; Otake et al., 2010). Because of advantages over subjective sleep diaries or logs, actigraphy has been cited as an important tool for use in follow-up studies to examine treatment efficacy and outcome (Ancoli-Israel). This is most relevant to this research study and purpose, as it relates to using actigraphy in assessing treatment outcome in sleep disorders; specifically, to evaluate the response of OSA patients to CPAP therapy, not in terms of determining respiratory events or isolating only sleep activity, but the assessment of total 24-hour sleep and wake activity combined with measures of CPAP compliance and energy expenditure.

Because assessing total energy expenditure is important to this study purpose, an actigraphy device that measures sleep activity, wake activity, and calculated total energy expenditure would be of primary interest. Respironics ${ }^{\circledR}$ markets both the Actiwatch ${ }^{\circledR}$ and Actical ${ }^{\circledR}$ device, which offered utility as measurement tools for this study. While the Actiwatch ${ }^{\circledR}$ has been used extensively in sleep research to measure sleep and wake activity, the Actical ${ }^{\circledR}$ has not been used to measure activity and energy expenditure in 
sleep, OSA, or CPAP patients. The intended use of the Actical®, as reported in premarket summary, is to serve as "a compact, lightweight, waist, wrist, or ankle worn activity monitor that may be used to assess human gross motor activity, caloric expenditure and estimates of energy expenditure based on motor activity in any instance where quantifiable analysis of physical motion is desirable" (Respironics, Inc $₫$, 2006, pp. 1-2). To date, the Actical ${ }^{\circledR}$ has been used in a variety of metabolic, behavioral, activity, and physical exercise research, dating back to 1998 , and has been validated in adult, child and adolescent populations (Mini Mitter ${ }^{\circledR}, 2003$ ). The device uses an accelerometer to provide measurement of acceleration in activity counts and calculation of activity energy (calorie) expenditure. Sleep software programs are available with the Actiwatch ${ }^{\circledR}$, and when combined with the Actical ${ }^{\circledR}$, these are ideal devices to measure variables of sleep and wake activity and energy (calorie) expenditure for this study.

\section{Summary}

The literature identifies sleep to have restorative powers to improve wakefulness, increase attention and memory, and promote higher level mental and physical functioning and performance (Carskadon \& Dement, 2005). Despite substantial sleep knowledge, there exists a pervasive failure to disseminate and translate existing knowledge and educate healthcare professionals and the public about sleep, sleep disorders and health (Walsh et al.; Institute of Medicine Committee on Sleep Medicine and Research Board on Health Sciences Policy, 2006). As a result, the numbers of those suffering with sleep disorders continues to grow, currently estimated at 50 to 70 million Americans, 18 million of whom are estimated to have obstructive sleep apnea (U.S. Department of 
Health And Human Services, National Institutes of Health, National Heart, Lung, and Blood Institute, \& National Center on Sleep Disorders Research, 2003).

OSA disrupts normal sleep architecture and physiology, resulting in many individual and public health risks (National Sleep Foundation, 2002; U.S. Department of Health And Human Services, National Institutes of Health, National Heart, Lung, and Blood Institute, \& National Center on Sleep Disorders Research). Such risks include hypertension, neuropsychologic and cognitive dysfunction, impotence, sleepiness related motor vehicle crashes, cardiovascular disease, and stroke (Phillips \& Kryger, 2005; Young, Peppard, \& Gottlieb, 2002). Additional OSA risks include T2DM associated with glucose intolerance/insulin resistance and metabolic syndrome, and increased leptin levels associated with obesity (Punjabi \& Beamer, 2005). Sleepiness, depression, decreased quality of life, and increased health care costs (Kapur et al., 1999; Won \& Robert, 2007) have been linked to OSA as well. Clearly, OSA patients are an at-risk population.

Although the benefits of nasal CPAP as an effective form of therapy for OSA has been well documented, treatment adherence and compliance remains problematic, despite known cardiovascular, metabolic and quality of life benefits (Basner, 2007; Grunstein, 2005; Phillips \& Kryger, 2005). While the data suggests favorable effects of CPAP, many questions and gaps remain including compliance, responsiveness to therapy and long-term benefits. Additional research addressing responsiveness to therapy in terms of sleep and wake activity and CPAP compliance would offer contribution toward filling the gaps in the current body of knowledge and state of sleep science. 
As OSA relates to overweight and obesity, there is a direct link to cardiovascular disease, hypertension, dyslipidemia, T2DM, cancer, osteoarthritis, pregnancy complications, and sleep apnea (Won \& Robert, 2007; Leinum, Dopp, \& Morgan, 2009). Findings have shown OSA to not only be a health risk factor associated with obesity, but an independent contributing factor to many of these same comorbidities (Dyken, Ali, Raman, \& Eppen, 2007; Leinum et al). Additionally, there is supportive evidence that metabolic and endocrine changes which promote obesity and diabetes may be a result of not only sleep disruption and arousal caused by OSA, but sleep loss and overall short sleep duration, as well (Spiegel, Leproult, \& Van Cauter, 1999; Hayes, Xu, Babineau, Patel, 2011). Given the present obesity trend and the strong relationship between obesity and OSA, health and healthcare costs can only be expected to rise exponentially without prioritizing public and individual healthy weight management and weight loss interventions. Research addressing the OSA population, $70 \%$ of whom are estimated to be obese, has such relevance and importance to both individual and public health.

The Actiwatch ${ }^{\circledR}$ and Actical ${ }^{\circledR}$ are ideal devices, and in combination have the ability to measure sleep activity, wake activity and calculated total energy expenditure,

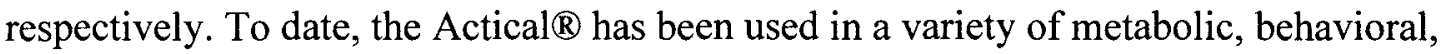
activity and physical exercise research, and has been validated in adult, child and adolescent populations (Mini Mitter $($ ), 2003). The device uses an accelerometer to measure both frequency and intensity of movement with activity counts and calculation of activity energy (calorie) expenditure. A software program is available for use with the Actiwatch ${ }^{\circledR}$ to download variables of sleep and wake activity. Likewise the Actical $®$, has a software program to download acquired data and determine energy (calorie) 
expenditure (Mini Mitter). Actigraphy, using the Actical ${ }^{\circledR}$ device, to evaluate EE in OSA patients has yet to be performed, but has implications for measuring energy expenditure and weight management in OSA patients. Combining the Actiwatch ${ }^{\circledR}$ and Actical ${ }^{\circledR}$ to study OSA patients in an unrestricted and natural environment, both prior to and following CPAP treatment, would contribute to measuring treatment efficacy, and provide data potentially useful to developing weight loss strategies in this population (Stenlof et al.).

Overweight and obesity is a major factor in OSA, contributing not only to its pathogenesis, but complicating its management and contributing to related comorbidities. Clinically, addressing obesity treatment presents challenges to OSA patients and health care providers, with little evidence supporting CPAP as a significant weight loss measure. This study aims to contribute to the current state of sleep science by addressing an important knowledge gap in the treatment of overweight and obese OSA patients; specifically, to assess the effect of CPAP treatment on 24-hour total sleep activity, wake activity and energy expenditure as measured in a patient's routine and usual environment, while accounting for CPAP compliance.

Current recommendations for research in OSA patients have highlighted the need for treatment outcome studies (Institute of Medicine Committee on Sleep Medicine and Research Board on Health Sciences Policy, 2006; U.S. Department of Health and Human Services, National Institutes of Health, National Heart, Lung, and Blood Institute, \& National Center on Sleep Disorders Research, 2003). While there is evidence supporting favorable effects and improvement in sleepiness, quality of life, and performance postCPAP therapy, the research does not address the effectiveness of CPAP in relation to 
improving overall objective measures of continuous 24-hour total sleep/wake activity and energy expenditure. To address this knowledge gap, the present study proposed the use of actigraphy as a practical, convenient, and cost-effective method to measure responsiveness to CPAP therapy in terms of sleep/wake activity and energy expenditure. Furthermore, findings from this study may offer important clinical, social, and economic implications associated with evaluating CPAP effectiveness, as well as identifying factors critical to understanding and developing weight loss strategies for the overweight, obese, and at-risk OSA population. 


\section{CHAPTER III}

\section{METHODS}

\section{Study Design}

This study employed a prospective, observational, longitudinal, and repeated measures study design, in which data were collected from a single sample of overweight and obese adults diagnosed with OSA who required CPAP therapy. Primary study outcomes evaluated changes in 24-hour total sleep activity, wake activity, and energy expenditure over time as measured via actigraphy. Data were collected at three fixed time points: prior to CPAP treatment (time point 1 [T1]), one week post-CPAP treatment (time point 2 [T2]), and 30 days post-CPAP treatment (time point 3 [T3]). At each time point, data were collected for 10 consecutive days and included 24-hour sleep activity, wake activity, and energy expenditure. Ten days was chosen to allow for the inclusion of both weekday and weekend data, providing a more comprehensive understanding of the effects of CPAP. Additionally, the pre-CPAP time point selection of a full week was based on the usual timeframe for clinical scheduling between consultation and diagnostic PSG. Post-CPAP time points were chosen to evaluate adjacent changes in the outcomes at the initial one-week pre-CPAP, as well as extended longitudinally to observe distant changes in the outcomes at the clinical standard of one month post-CPAP. In addition, prior research concerning compliance data has shown that CPAP use is often discontinued within the first one to two weeks after beginning treatment (Grunstein, 2005); therefore, the one week post-CPAP was a critical time point for data collection 
and provided the opportunity to collect data from those individuals who did not continue therapy through the 30-day time point. Furthermore, because CPAP compliance at one month has shown to be predictive of compliance at 3 months, additional time points were not included in the current study (Kribbs et al., 1993; Weaver et al., 1997).

\section{Sample and Setting}

The target population for this study was overweight and obese adults diagnosed with OSA. Participation was dependent upon a subject's eligibility, willingness to participate, and signed informed consent. Interested subjects were required to meet the following inclusion criteria: 1) male or female; 2) adults age 18 to 65 ; 3) able to speak, read and write the English language; 4) overweight or obese at baseline (BMI $\geq 25) ; 5$ ) initially present with signs and symptoms consistent with OSA and diagnostically confirmed with an $\mathrm{AHI} \geq 5$ as determined by PSG; 6) require CPAP therapy (physicianprescribed); and 7) maintain a regular sleep schedule, going to bed routinely between the hours of 9:00 pm and 1:00 am and allowing 6 to 9 hours of sleep time. Likewise, potentially eligible subjects were excluded if they met any of the following exclusion criteria: 1) non-traditional work hours (night shift or swing shift work); 2) any known acute, unstable, or untreated medical conditions; and 3) pregnant or planning to become pregnant.

Children and older adults have different sleep architecture and sleep requirements when compared to adults aged 18 to 65 (Carskadon \& Dement, 2005); therefore, it has been recommended that children and adults older than 65 should be studied independently, and, as a result, were not recruited for the current study. This research did not pose a pregnancy risk; however, because both obesity and OSA may be affected 
during pregnancy, pregnant women were excluded from study participation. Night shift and swing shift workers were not eligible for study participation due to the interrupted sleep/wake schedules involved in these types of shift work. Those with acute, unstable, or untreated medical conditions were not included in the study because these conditions could have affected their ability to undergo sleep study procedures and CPAP treatment. Minorities were not excluded from study participation.

The sample population obtained for analysis represented a convenience sampling of patients evaluated at Sleep Medicine Specialists (SMS), a private physician practice in Louisville, Kentucky. Population demographics were evaluated prior to data collection and estimated to be $80 \%$ adult (ages 18 to 65 ), $75 \%$ male, $85 \%$ Caucasian, $75 \%$ privately (non-state funded) health insured, and less than $1 \%$ uninsured. Approximately $85 \%$ of SMS patients are either self- or physician-referred for evaluation of OSA. On average, there are a total of 25 to 30 new sleep patients evaluated each week for OSA; therefore, subject recruitment and enrollment from this single site seemed feasible to adequately power this study. SMS is a practice of eight pulmonary physicians, all of whom are board certified in sleep medicine. In this setting, patients are initially seen for consultation in an outpatient physician office and subsequently scheduled for overnight testing in the sleep center. The sleep center is located adjacent to the physician offices. SMS maintains accreditation by the American Board of Sleep Medicine, and has a total of 10 diagnostic sleep rooms. The sleep rooms are appointed with standard or queen size beds, overhead lighting, bedside table, lamp, cable television, sink, and a chair. Restrooms with showers are located adjacent to sleep rooms. 


\section{Human Subjects Considerations}

The protocol and informed consent form (ICF) were approved by the University of Louisville Human Subjects Protection Program Office (HSPPO). Approval letters can be found in Appendix A. Subject recruitment material was not needed for subject enrollment. Eligible men and women, ages 18-65, as well as English-speaking minorities from the SMS practice, were recruited for this study.

The ICF process was conducted by the investigator in a private exam room, and included a discussion and explanation of the study purpose and goal, study procedures, type of data collected, conflicts of interest, study funding or sponsorship, risks and benefits associated with the study, alternative procedures or treatment of OSA, compensation, study personnel contact information, and relevant patient confidentiality and health information protection and privacy issues (Polit \& Beck, 2004). It was expected that study-related procedures would involve minimal risk to subjects. Emphasis was placed on the voluntary nature of study participation, and that the subject may withdraw at any time with no fear of their withdrawal from the study affecting their treatment. The subjects were informed that compensation would not be provided for study participation. Human subject protection, confidentiality, and health information privacy was also incorporated into the ICF and reviewed with study participants. Following a discussion, subjects were allowed adequate time to read the ICF and ask questions. All questions were answered by the investigator to the subject's satisfaction. No data collection or other study-related procedures were performed prior to the study participant signing ICF, and a signed ICF copy was provided to all subjects. 
There were no conflicts of interest to be disclosed. Specifically, there was no compensation provided by the investigator to the referring SMS physician, nurse, clinical staff, or office practice. The investigator was not a clinical practitioner or an employee of SMS. The physicians, nurses, clinical staff, and investigator followed professional and ethical conduct and did not use coercive tactics for subject recruitment. Additionally, the SMS clinical staff and physician practice were not involved in data collection or analysis of the research.

\section{Measurement}

This study focused on changes in wake activity (time in minutes), sleep activity (time in minutes), and total energy (kilocalorie) expenditure over time, both prior and subsequent to CPAP therapy. The Actiwatch ${ }^{\circledR}-64$ and Actical ${ }^{\circledR}$ devices were the instruments used to obtain these measurements. Philips-Respironics, Inc. provided 10 Actiwatch ${ }^{\circledR}$ and Actical ${ }^{\circledR}$ monitoring devices, the ActiReader hardware, corresponding software programs, and instruction manuals for each monitoring system. As referenced in the literature review, most studies have consistently shown actigraphy to be a valid assessment of sleep patterns with significant correlation to PSG in measuring total sleep time (Matsumoto et al., 1998; Hedner et al.; Vallieres \& Morin, 2003). Advantages of actigraphy include its' cost-effectiveness and ability to non-invasively and objectively record continuous physical movements during sleep and wake activity in a natural environment, over extended periods of time (Ancoli-Israel, 2005; Kushida et al., 2001). Variables of interest to this study can be referenced in Table 1, with detailed discussion of each of these variables provided within separate sections labeled as outcome variables, 
Table 1

Variables and Measurement

\begin{tabular}{ll}
\hline Variable & Data Collection Instrument \\
\hline Sleep activity (in minutes) & Actiwatch ${ }^{\circledR}$ \\
Wake activity (in minutes) & Actiwatch ${ }^{\circledR}$ \\
Energy expenditure (in kcals) & Actical ${ }^{\circledR}$ \\
Gender & Clinical Chart - Self-Report Questionnaire \\
Age & Clinical Chart - Self-Report Questionnaire \\
Race & Clinical Chart - Self-Report Questionnaire \\
Education Level & Clinical Chart - Self-Report Questionnaire \\
Work Status & Clinical Chart - Self-Report Questionnaire \\
Work Schedule & Clinical Chart - Self-Report Questionnaire \\
Sleep Schedule & Clinical Chart - Self-Report Questionnaire \\
Marital Status & Clinical Chart - Self-Report Questionnaire \\
Height & Clinical Chart \\
Weight & Clinical Chart \\
Body Mass Index & Clinical Chart \\
Child-bearing Potential Status & Clinical Chart/Urine Pregnancy Test \\
Medical History & Clinical Chart - Self-Report Questionnaire \\
Apnea-Hypopnea Index & Clinical Chart - Polysomnographic Report \\
CPAP Pressure & Clinical Chart - CPAP Report \\
CPAP Compliance & EncorePro ${ }^{\circledR}$ SmartCard $®$ \\
\hline
\end{tabular}


demographic variables, OSA and CPAP compliance variables, and height, weight, body mass index and pregnancy variables.

\section{Outcome variables}

Sleep, wake, and energy expenditure (EE) were the outcome variables of interest in this study and were measured using two wrist-worn actigraphy devices. The Actical $\mathbb{B}_{\text {, }}$ as reported in premarket summary, is intended to serve as "a compact, lightweight, waist,wrist, or ankle worn activity monitor that may be used to assess human gross motor activity, caloric expenditure and estimates of energy expenditure based on motor activity in any instance where quantifiable analysis of physical motion is desirable" (Respironics, Inc $®, 2006$, pp. 1-5). This device uses an omni-directional piezoelectric accelerometer to provide measurement of physical motion. The degree of speed and motion produces voltage reading which is stored as an activity count. Activity counts have cut-point intensity values and corresponding algorithms for calculating activity energy expenditure

$\left(\mathrm{kcal} \cdot \mathrm{kg} \cdot \mathrm{min}^{-1}\right)$. The Actical ${ }^{\circledR}$ predicts EE and utilizes the Actical ${ }^{\circledR}$, Version 2.10 software to download data via the ActiReader ${ }^{\circledR}$ device (Respironics, 2007). An example of Actical $®$ data can be found in Appendix B.

To date, the Actical ${ }^{\circledR}$ has been used in a variety of metabolic, behavioral, activity and physical exercise research, and has been validated in adult, child, and adolescent populations (Mini Mitter ${ }^{\circledR}, 2003$ ). Heil (2006) developed equations for predicting activity energy expenditure (AEE) in children and adults using the Actical® device. This laboratory-based study resulted in regression models with subsequent algorithms for predicting AEE based on physical activity intensity at three monitoring locations (hip, ankle and wrist). Heil concluded that these generalized prediction equations and 
corresponding algorithms would provide useful assessment of time-based physical activity in groups of children and adults, but would likely never "accurately predict AEE for all activities in all people" (p. 76). Heil suggested monitors that allow for individual calibration could overcome this limitation; however, such a monitor has yet to be developed. Heil further reported physical activity and AEE measurement was sensitive to the body part that is responsible for the motion and that placement of the Actical® limits the precision of measurement when worn in a single location. In adults, both modeling algorithms accurately predicted light and moderate activities in all monitor locations but was most consistent for vigorous activity in the hip location.

Crouter, Churilla \& Bassett (2006) conducted a free-living study to examine the validity of predicted $\mathrm{EE}$ regression equations from three accelerometers (including the Actical $($ ). These researchers compared the three accelerometers to indirect calorimetry across a range of sedentary to vigorous physical activities. Findings revealed that no single regression equation offered precise determination of the time spent in a specific activity intensity or an accurate prediction of corresponding EE.

Rothney, Shaefer, Neumann, Choi and Chen (2008) also conducted a free-living study to validate EE predications from three different commercially available accelerometers, including the Actical ${ }^{\circledR}$. These authors found less than $2 \%$ differences in moderate and vigorous physical activity prediction equations of the accelerometers as compared to room calorimeter measurements. Sedentary and light intensity activities tended to either over- or underpredict EE (Rothney et al.). This study offered similar conclusions as Heil (2006) and Crouter, et al. (2006), indicating the need for developing new regression equations to establish more accurate predictions of physical activity 
intensity. Lastly, a study conducted by Feeney et al. (2007), addressed the validation of the Actical ${ }^{\circledR}$ in obese and non-obese middle-aged adults. This study found differences in currently established AEE and activity count cut-points when comparing the younger non-obese and middle-aged obese study participants. The results of this study suggested the need to establish specific age and body composition algorithms for measuring physical activity and EE with the Actical ${ }^{\circledR}$ accelerometer (Feeney, et al.).

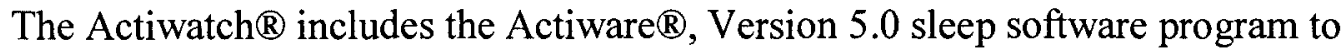
allow sleep and wake activity data to be downloaded via the ActiReader ${ }^{\circledR}$ and measured in time (Respironics, 2006). The complex algorithms for scoring and calculating data have been validated and score sleep and wake based on sampled epochs (15 second, 30 second, 1 minute, or 2 minute) of activity counts. These activity counts are scored as either sleep or wake depending on whether the number of counts exceeds or falls below the established intensity thresholds set for sleep and wake. An example of the Actiwatch ${ }^{\circledR}$ data can be found in Appendix C. Like the Actical ${ }^{\circledR}$, the Actiwatch ${ }^{\circledR}$ uses an accelerometer with a sensitivity of less than $.01 \mathrm{~g}$-force to measure physical movement and produce an electrical current. The intensity of this electrical current is stored as an activity count and distinguishes wake, sleep, sleep onset, awakenings during sleep, and total sleep time (Ancoli-Israel, 2005). Kushida et al. (2001) compared actigraphic, polysomnographic, and subjective assessment of sleep parameters in sleep-disordered patients. Data for number of awakenings, total sleep time, and sleep efficiency were compared. Sensitivity, specificity and accuracy were calculated using the low- (highwake-sensitivity), medium- (medium-wake-sensitivity) and high-(low-wake-sensitivity) threshold algorithms from the Actiware ${ }^{\circledR}$ software program and compared to PSG and 
questionnaire data. Findings from this study were comparable to an earlier study by Babin, Lee, Halko, Boudreau and George (1997) revealing higher sensitivity (0.98 actigraphic ability to detect sleep) for measuring sleep parameters in sleep disordered patients with the higher threshold algorithm at the cost of lower accuracy $(0.80-$ actigraphic ability to detect both sleep and wake) and specificity (0.36 - actigraphic ability to detect wake). Overall, the accuracy of actigraphy in patients with sleep disorders has been reported to range between 0.78 and 0.85 (Blood, Sack, Percy $\&$ Pen, 1997; Oakely, 1997; Sadeh, Hauri, Kripke \& Lavie, 1995).

While both the Actical ${ }^{\circledR}$ and Actiwatch have limitations in precision and accuracy associated with the individual nature of age, body composition, and free-living differences, each instrument offers validated measurements taken as a group. As such, the use of these instruments addresses a gap in the literature by combining measures of the Actiwatch ${ }^{\circledR}$ and Actical ${ }^{\circledR}$ device to measure the influence of CPAP on sleep/wake activity and energy expenditure in the overweight and obese OSA study population.

\section{Demographic variables}

Demographic variables included gender, age, race, education level, work status and schedule, sleep schedule, marital status, height, weight, BMI, child-bearing potential status, and medical history. Medical history data relevant to OSA comorbid conditions included hypertension, myocardial infarction, heart failure, cerebrovascular accident, dyslipidemia, diabetes, and depression. A category to capture other medical conditions was included, as well. Some of these demographic variables were used to ensure appropriate subject selection based on established eligibility criteria. Otherwise, demographics were used to describe the sample population. Contact information (name, 
date of birth, home address, and phone numbers) for each subject was collected in the event the investigator needed to reach the subject. This information (see Appendix D) was maintained separately from the subject's research data and stored in the coinvestigators secured, limited-access research office.

\section{OSA and CPAP compliance variables}

Polysomnography (PSG) was conducted as a routine clinical procedure for the diagnosis of OSA and CPAP titration. An AHI was obtained from the overnight PSG report on all subjects to determine if they met eligibility ( $\mathrm{AHI} \geq 5$ ). For those eligible subjects requiring CPAP, a physician-prescribed CPAP pressure (centimeters of water pressure) was collected from the CPAP titration PSG study report (filed in the clinical chart). PSG acquisition, diagnostic scoring, and CPAP titration were conducted following standard clinical and technical procedures for an accredited sleep center. All clinical PSG diagnostic and CPAP titration studies were reviewed and interpreted by a SMS board certified sleep physician. A sample of OSA and CPAP titration in raw data PSG form can be referenced in Appendices $\mathrm{E}$ and F. Additionally, an example of both diagnostic and CPAP titration PSG reports can be found in Appendices $\mathrm{G}$ and $\mathrm{H}$.

CPAP compliance data was obtained from standard CPAP machines equipped with meters recording CPAP use on a removable card, which was downloaded by the coinvestigator into a computer software program to generate compliance (hours of usage) reports (see Appendix I). The CPAP machines prescribed from the SMS practice are manufactured by Philips-Respironics and incorporate the Encore ${ }^{\circledR}$ Pro SmartCard $\mathbb{}$ technology to monitor and record the date, time and duration of CPAP use over a six month time period (Respironics, 2009). The Encore $®$ Pro software allows the 


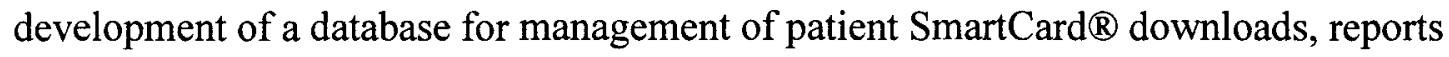

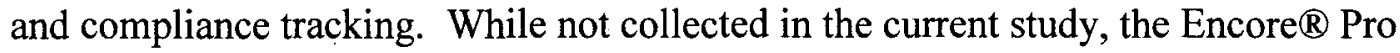
system further records data on pressure trends, tidal volume, respiratory rate, triggered breathing percentage, minute ventilation, air leaks, and alarms (Respironics, 2009). Height, weight, body mass index and pregnancy variables

Height and weight, with a calculated BMI were measured by the clinical staff and obtained from the subject's chart. These variables served as measures for determining study eligibility based on overweight or obese criteria, as well as for descriptive purposes. BMI was calculated by the SMS clinical staff, but was confirmed by the research co-investigator using the standard formula: weight in kilograms divided by height in meters squared. To meet study eligibility criteria, as required by the local HSPPO, a urine pregnancy test was conducted on all females of child-bearing potential (females were considered to be of child-bearing potential unless they reported spontaneous amenorrhea for at least 12 months or reported having had a hysterectomy and/or bilateral oophorectomy) to confirm negative urine human chorionic gonadotropin (HCG).

\section{Data Collection Procedures}

Subjects were recruited on a rolling basis from SMS over a 14 month time period, from November 2009 to January 2011. Clinical patients undergoing initial OSA evaluation at SMS were informed of the opportunity to be involved in a research study. All sleep nurses and physicians (clinical staff) at SMS were provided information about the research study and asked to identify potentially eligible subjects. A visit schedule of procedures can be referenced in Appendix $\mathrm{J}$ and data collection forms for all visits can be 
found in Appendix K. All study procedures, with the exception of the urine pregnancy test and actigraphy (Actiwatch $囚$ and Actical $\circledast$ ) training/instruction, monitoring, dispensing/return, and download, were routine standard of care for SMS.

Visit 1.0

Once a subject was deemed eligible and expressed an interest in participating, the investigator approached the patient to discuss the study purpose and procedures, privacy and protection of health information, and the risks and benefits of study participation. Upon the subject's verbal understanding and written signed/dated informed consent, the investigator proceeded with initial data collection from the subject's clinical chart, including initials, gender, age, race, education level, work status and schedule, sleep schedule, marital status, height, weight, BMI, child-bearing potential status, and medical history (hypertension, myocardial infarction, heart failure, cerebrovascular accident, dyslipidemia, diabetes, depression, and other). Contact information (home address and phone numbers) for each subject was also collected and maintained in a separate subject identification log. This information was used to communicate with the subjects and was maintained in the investigator's secured, limited-access research office.

To meet the local HSPPO's requirements, a urine pregnancy test was collected on all females of child-bearing potential to determine pregnancy status during the first visit. Child-bearing information was obtained from all female subjects by the investigator to determine the need to collect urine pregnancy test. The subject's clinical chart was reviewed during Visit 1.0 to determine if the subject met study eligibility. Missing or unclear demographic information, relevant to the study, was clarified by the investigator during this visit. 
The subject was then instructed on the use of the Actiwatch $\circledast$ and Actical $\AA$ device and asked to wear both wrist devices 24 hours a day (without removing) for 10 days prior to their scheduled diagnostic PSG (one week pre-CPAP data collection). Based on findings from an earlier unpublished pilot study, a 10 day actigraphy data collection period was chosen in order to collect a full week (seven days) of continuous 24 hour data (including five weekdays, Monday through Friday, and two weekend days, Saturday and Sunday) (McCullough, 2008). This pilot study collected actigraphy data for seven days but yielded only five days of evaluable data with the starting day (Day 1) and ending day (Day 7) days having partial data (less than 24 hours).

\section{Visit 2.0 and 2.1}

At Visit 2.0, subjects returned for overnight PSG, which was either a diagnostic PSG with a second CPAP treatment/titration PSG, or a single split diagnostic PSG and CPAP treatment/titration PSG (split night study). Split night studies are determined by sleep time and OSA severity per health insurance/medical coverage plans. Whether a subject underwent a single split diagnostic/treatment night (Visit 2.0) or two nights for diagnosis and treatment (Visit 2.1) could be considered a potential confounding factor in the final data analyses. Those study participants who required 2 nights for diagnosis and treatment experienced a lapse of time between the diagnostic PSG (Visit 2) and the CPAP treatment/titration PSG (Visit 2.1). This lapse of time was one to two weeks, depending on the clinical schedule. The AHI $(\geq 5)$ and prescribed CPAP pressure were collected from the PSG report filed in the clinical chart. All PSG acquisition and scoring, as well as CPAP titration, were conducted by a trained SMS PSG technologist and interpreted by a SMS board certified sleep physician. 
During Visit 2.0, actigraphy data download from both the Actical ${ }^{\circledR}$ and Actiwatch $₫$ was conducted. All eligible subjects were scheduled by the SMS office staff to return for a routine CPAP set-up office visit. Additional data collected at this visit included any reported adverse events related to study participation and/or procedures. Visit 3.0

Subjects returned for a routinely scheduled clinical office visit at SMS for CPAP set-up. The durable medical equipment company (DME) provided the PhilipsRespironics CPAP equipment, compliance SmartCard $®$, and necessary training and instruction on home use of the equipment, as usual and customary. During Visit 3.0, the investigator saw the subject and provided instruction and training on the use of the Actical ${ }^{\circledR}$ and Actiwatch $®$ devices. The actigraphy devices were dispensed to the subject and they were asked to again wear both wrist devices 24 hours a day (without removing) for approximately forty days starting on the first day of CPAP therapy at home (in order to obtain both the one week and one month post-CPAP time points for data collection). Additional data collected at this visit included any reported adverse events related to study participation and/or procedures.

\section{Phone Contact}

Subjects were contacted by the investigator (by phone) approximately 10 to 14 days following the first day of CPAP use at home. This phone contact served to assess the subject's use of the Actical ${ }^{\circledR}$ and Actiwatch ${ }^{\circledR}$ devices and CPAP therapy. Additional data collected at this visit also included any reported adverse events related to study participation and/or procedures. 
Visit 4.0

Subjects returned for a regularly scheduled clinical office visit at SMS after approximately one month of CPAP treatment home. During Visit 4.0, the investigator saw the subject and performed a CPAP compliance data download and an actigraphy data download from both the Actical ${ }^{\circledR}$ and Actiwatch ${ }^{\circledR}$ devices. Additional data collected at this visit included any reported adverse events related to study participation and/or procedures. This visit concluded study participation for the subject.

With the exception of PSG acquisition and scoring and CPAP titration, all data collection and procedures involving the Actiwatch $®$ and Actical ${ }^{\circledR}$ devices and related subject visits were conducted by the co-investigator. Total subject duration in the study was dependent on clinical diagnostic and treatment scheduling with an expected duration of about two months.

The initial office visit, PSG, CPAP titration, CPAP machine/supplies/compliance card, and any associated education or instruction is considered standard care for OSA patients and was subject to usual healthcare billing procedures by SMS and the DME company. The training and use of the Actiwatch $®$ and Actical $®$ device did not fall under usual care and was considered a study-related procedure. Subjects were not compensated for any standard of care procedures or study-related procedures.

Data Management Procedures

Unique identifiers were assigned to each subject to maintain anonymity (except to those directly involved in the research). Each subject had a 3 digit number consecutively assigned beginning with 001 . All study participant information was stored in the 
investigator's secured, limited-access research office. Furthermore, all research data were collected, maintained, and entered for statistical analysis by the investigator.

Statistical Analysis

Descriptive analysis

Data analyses were performed using SPSS version 17.0 (SPSS, 2008). Study demographics were obtained for the entire sample, and descriptive statistics were calculated. Differences in demographic variables between those subjects who completed all study time points and those who did not complete all study time points were tested using $t$-test for continuous variables and Chi-square tests for categorical variables. The analytic sample was then dichotomized into CPAP compliant $(\geq 4$ hours/night on $70 \%$ of the observed nights) and CPAP noncompliant ( $<4$ hours/night on $70 \%$ of the observed nights) to test for differences between these two groups. These differences were tested using Chi-square techniques for all categorical data and $t$-tests for all continuous variables, utilizing an alpha of 0.05 .

Inferential analysis

Three separate two-factor repeated measures analysis of variance (ANOVA) models were developed to detect overall group differences between pre-CPAP (T1), 1 week post-CPAP (T2), and 30 days post-CPAP (T3) on 24-hour sleep, wake, and energy expenditure. Repeated measures ANOVA allowed for group comparisons of the three study variables at different points over time (Munro, 2005), specifically testing for main effects for group (CPAP compliant versus CPAP non-compliant) and time. Utilizing repeated measures offered the ability to decrease the error term, while requiring fewer subjects to increase the power of the analysis (Munro). Although this study did not 
involve a random sample, the research met other ANOVA assumptions, which included continuous dependent variable observed across mutually exclusive groups (the dependent variable observations are independent) and equal variances (homogeneity) across the groups on the dependent variable (observations on the dependent variable follow a normal distribution in each group) (Stevens, 2002).

While repeated measures ANOVA is an omnibus test, post-hoc comparisons are needed if an effect is seen for variables with more than two levels (e.g., three time points). As such, paired $t$-tests techniques were performed to test the sub-hypotheses for differences between two time points. The analyses conducted allowed for exploration of the following hypothesized outcomes (hypothesis 1 [H1], hypothesis 2 [H2], hypothesis 3 [H3]):

H1) Subjects will have a significant decrease in total 24-hour sleep activity over time as a result of using CPAP (measured by the Actiwatch $($ )

Hla. compared to pre-CPAP, there will be a significant decrease in 24-hour total sleep activity (time in minutes) 7 days post-CPAP H1b. compared to pre-CPAP, there will be a significant decrease in 24-hour total sleep activity (time in minutes) 30 days post-CPAP

H2) Subjects will have a significant increase in total 24-hour wake activity over time as a result of using CPAP (measured by the Actiwatch $®$ ) H2a. compared to pre-CPAP, there will be a significant increase in 24-hour total wake activity (time in minutes) 7 days post-CPAP H2b. compared to pre-CPAP, there will be a significant increase in 24-hour total wake activity (time in minutes) 30 days post-CPAP 
H3) Subjects will have a significant increase in energy expenditure over time as a result of using CPAP therapy (measured by the Actical ${ }^{\circledR}$ )

H3a. compared to pre-CPAP, there will be a significant increase in 24-hour total energy (kilocalorie) expenditure 7 days post-CPAP

H3b. compared to pre-CPAP, there will be a significant increase in 24-hour total energy (kilocalorie) expenditure 30 days post-CPAP

The first hypothesis 1 (H1) examined the effect CPAP had on sleep activity; H2 examined the effect CPAP had on wake activity; and H3 explored the effect CPAP had on energy expenditure. For each outcome, separate repeated measures ANOVA models were developed.

\section{Sample size calculation}

An a priori statistical power analysis was conducted using the $\mathrm{G}^{*}$ power 3.0 program to determine the number of subjects required to adequately power the repeated measures ANOVA analysis and study design (Faul, Erdfelder, Lang, \& Buchner, 2007). The program's field options were populated to include a single group, within factor ANOVA with 3 time points. Because the magnitude of the relationship (treatment effect) of CPAP treatment on sleep/wake activity and energy expenditure has not been well established in prior research, the estimated effect size used for the sample size calculation was set at a 0.25 , defined as a moderate effect size using Cohen's $d$ (Cohen, 1988). The effect size chosen is also consistent with effect sizes routinely reported in the social sciences and nursing research (Polit \& Beck, 2008; Cohen). Additionally, correlation occurs when repeated measures are obtained from the same individual over time, with adjacent time points being more correlated than distant time points. Therefore, the 
correlation values were set at 0.5 for adjacent time points $(\mathrm{T} 1, \mathrm{~T} 2$ and $\mathrm{T} 2, \mathrm{~T} 3)$ and 0.25 for the distant time points $(\mathrm{T} 1, \mathrm{~T} 3)$. The desired power was set at 0.80 with a 0.05 test significance level. Using these parameters, a sample size of 34 was needed to achieve an actual power of 0.81 . That is, to adequately power the current study, 34 subjects were needed to complete all three data collection time points.

In order to obtain a final sample size of 34 , an attrition rate had to be anticipated. It has been reported that $58 \%$ to $80 \%$ of patients either initially refuse or do not continue CPAP treatment following a trial (Basner, 2007; Grunstein, 2005); however, objective monitoring of CPAP compliance has more accurately revealed about $46 \%$ of OSA patients used CPAP 4 hours or more on at least $70 \%$ of the observed nights, with compliance at one month predictive of compliance at 3 months (Kribbs et al., 1993; Weaver et al., 1997). Using a 54\% attrition rate to conservatively estimate total sample size, it was projected that 74 subjects needed to be recruited and enrolled in order to obtain $n=34$ at $\mathrm{T} 3$. The observed attrition rate was slightly less than $54 \%$, requiring only 69 enrolled subjects (51\% attrition). Study recruitment ceased once 34 individuals completed all three data collection time points. All remaining enrolled subjects were allowed to continue through all study protocol procedures, with a total of 35 subjects completing all three time points. The enrollment period for this study was slightly over 14 months. The first subject was consented November 17, 2009, and the final subject completed study participation on January 27, 2011. 


\section{CHAPTER IV}

\section{RESULTS}

A total of 69 subjects were initially consented and enrolled into the current study.

Of the 69 consented, six did not complete a diagnostic sleep study (PSG) and six did not demonstrate an $\mathrm{AHI} \geq 5$. The remaining 57 subjects qualified for CPAP treatment; however, seven did not elect this form of therapy, leaving 50 eligible study participants completing pre-CPAP time point $1(n=50)$. Twelve $(24 \%)$ of these 50 subjects did not complete the one week post-CPAP time point $(\mathrm{n}=38)$ and 3 did not complete the one month post-CPAP time point $(n=35)$. Figure 2 (Study Flow Diagram) illustrates the study enrollment and participation process.

\section{Descriptive Statistics}

\section{Eligible sample}

Demographic variables for the eligible subjects $(n=50)$ were obtained and can be referenced in Tables 2 and 3. The mean age for the eligible sample was 49.3 years and $52 \%$ were male. The mean body mass index (BMI) was 37.5 , the average apneahypopnea index was 26.9 , and the mean CPAP pressure was $9.96 \mathrm{~cm} \mathrm{H}_{2} 0$. The sample was $82 \%$ Caucasian, with African-American comprising the remaining 18\%. More than one-third (38\%) of the sample reported a high school education level, with $28.0 \%$ reporting a college graduate level (16 years of education). Only $2 \%$ had less than 12 
Figure 2. Study Flow Diagram.

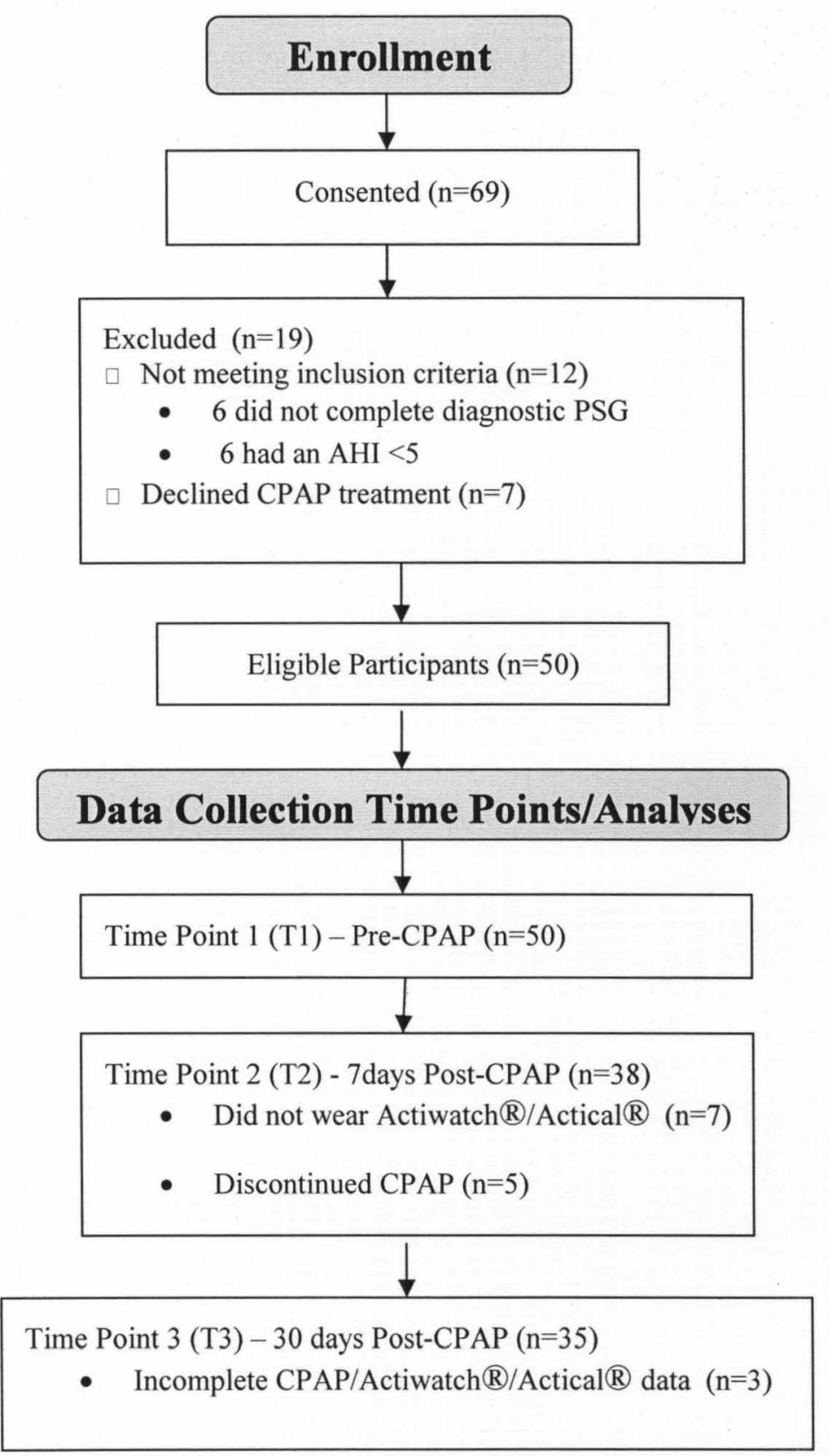


Table 2

Descriptive Data of Eligible Subjects - Continuous Variables

\begin{tabular}{lccc}
\hline $\begin{array}{l}\text { Variable } \\
\text { Eligible Subjects }(\mathbf{n = 5 0})\end{array}$ & Minimum & Maximum & Mean (SD) \\
\hline Age & 28 & 65 & $49.3(10.47)$ \\
BMI & 27.3 & 60.9 & $37.5(7.41)$ \\
AHI & 5.3 & 135.3 & $26.9(24.49)$ \\
CPAP Pressure & 6.0 & 16.0 & $9.9(2.91)$ \\
\hline
\end{tabular}


Table 3

Descriptive Data of Eligible Subjects - Categorical Variables

Variable

Gender

Male

Female

Ethnicity

Caucasian

African American

\section{Education}

$<12$ years

High School

Tech/Vocational

College Grad (14yrs)

College Grad (16yrs)

College Grad (18yrs)

\section{Eligible Subjects}

$\mathrm{n}=50(\%)$
$26(52.0 \%)$

$24(48.0 \%)$

$41(82.0 \%)$

$9(18.0 \%)$

\section{Marital Status}

Single

$11(22.0 \%)$

Married

$31(62.0 \%)$

Divorced

$5(10.0 \%)$

Widowed

$1(2.0 \%)$

Separated

$2(4.0 \%)$

\section{Work Status}

Unemployed

$6(12.0 \%)$

Part-time

$7(14.0 \%)$

Full-time

$29(58.0 \%)$

Disabled

$5(10.0 \%)$

Retired 
years of education and $8 \%$ reported post-graduate education. The majority were married (62\%), with $22 \%$ single, $10 \%$ divorced, $2 \%$ widowed, and $4 \%$ separated. Work status revealed $58 \%$ full-time workers, $14 \%$ part-time workers, $12 \%$ unemployed, $10 \%$ disabled, and $6 \%$ retired. Hypertension was the most frequently reported medical condition by 21 subjects, followed by 11 reporting dyslipidemia. A list of additional medical conditions reported by the sample of eligible subjects has been summarized in Table 4. There were seven subjects who had reported no medical history.

\section{Completed and early-term sample comparisons}

The sample $(n=50)$ was stratified into completed subjects $(n=35)$ and early-term subjects $(n=15)$. Completed subjects $(n=35)$ were those participants who completed all three time points (T1, T2, and T3) for the study. Early-term subjects ( $\mathrm{n}=15)$ were those who completed only the first two time points (T1 and T2). To determine if demographic differences existed between these groups, $t$-tests were performed on all continuous variables and Chi-square techniques on all categorical data, with the significance test level at 0.05 (see Table 5).

Of the eligible study participants $(n=50)$, the completed subjects were significantly older $(p=.026)$ than the early-term subjects (51.4 versus 44.3 years). BMI was also statistically different $(p=.013)$, with those completing the study having a lower mean BMI (35.8 versus 41.3). The completed subjects group included more males $(57.1 \%)$ than the early term subjects $(40 \%)$, but was not found to be significantly different. Both groups were predominately Caucasian. The average AHI (22.9) and CPAP pressure (9.7) was lower for those who completed compared to those who did not (36.1 and 10.4, respectively), but was not significantly different. The majority of each of 
Table 4

Medical Conditions Reported by Eligible Subjects

\begin{tabular}{lc}
\hline Medical History & $\begin{array}{c}\text { Eligible Subjects } \\
\mathbf{n}=\mathbf{5 0}(\mathbf{\%})\end{array}$ \\
\hline Hypertension & $21(42 \%)$ \\
Dyslipidemia & $11(22 \%)$ \\
Diabetes & $9(18 \%)$ \\
Depression & $9(18 \%)$ \\
Chronic Pain & $9(18 \%)$ \\
Gastric Reflux & $6(12 \%)$ \\
Arthritis & $6(12 \%)$ \\
Asthma/Allergies & $3(6 \%)$ \\
Polycystic Ovaries & $2(4 \%)$ \\
Seizure Disorder & $1(2 \%)$ \\
Heart Attack & $1(2 \%)$ \\
Heart Failure & $1(2 \%)$ \\
Stroke & $7(14 \%)$ \\
Migraines & $1(2 \%)$ \\
Celiac Disease & $9(4 \%)$ \\
\hline
\end{tabular}


Table 5

Demographic Data Comparison of Completed and Early-Term Subjects

\begin{tabular}{|c|c|c|c|}
\hline Variable & $\begin{array}{c}\text { Completed Subjects } \\
\mathbf{n}=35(\%)\end{array}$ & $\begin{array}{c}\text { Early-Term Subjects } \\
n=15(\%)\end{array}$ & p-value \\
\hline Age & $51.4(10.62)$ & $44.3(8.45)$ & $.026^{*}$ \\
\hline $\begin{array}{r}\text { Gender } \\
\text { Male }\end{array}$ & $20(57.1 \%)$ & $6(40.0 \%)$ & .266 \\
\hline $\begin{array}{l}\text { Ethnicity } \\
\quad \text { Caucasian }\end{array}$ & $31(88.6 \%)$ & $10(66.7 \%)$ & .065 \\
\hline $\begin{array}{l}\text { Education } \\
<12 \text { years } \\
\text { High School } \\
\text { Tech/Vocational } \\
\text { College Grad (14yrs) } \\
\text { College Grad (16yrs) } \\
\text { College Grad (18yrs) }\end{array}$ & $\begin{array}{c}1(2.9 \%) \\
15(42.9 \%) \\
1(2.9 \%) \\
9(25.7 \%) \\
7(20.0 \%) \\
2(5.6 \%)\end{array}$ & $\begin{array}{c}0(0.0 \%) \\
4(26.7 \%) \\
4(26.7 \%) \\
5(33.3 \%) \\
0(0.0 \%) \\
2(13.3 \%)\end{array}$ & $\begin{array}{l}.508 \\
.279 \\
.010^{*} \\
.582 \\
.062 \\
.363\end{array}$ \\
\hline $\begin{array}{l}\text { Marital Status } \\
\text { Single } \\
\text { Married } \\
\text { Divorced } \\
\text { Widowed } \\
\text { Separated }\end{array}$ & $\begin{array}{c}7(20.0 \%) \\
22(62.9 \%) \\
3(8.6 \%) \\
1(2.9 \%) \\
2(5.7 \%)\end{array}$ & $\begin{array}{c}4(26.7 \%) \\
9(60.0 \%) \\
2(13.3 \%) \\
0(0.0 \%) \\
0(0.0 \%)\end{array}$ & $\begin{array}{l}.602 \\
.849 \\
.607 \\
.508 \\
.345\end{array}$ \\
\hline $\begin{array}{l}\text { Work Status } \\
\text { Unemployed } \\
\text { Part-time } \\
\text { Full-time } \\
\text { Disabled } \\
\text { Retired }\end{array}$ & $\begin{array}{c}4(11.4 \%) \\
5(14.3 \%) \\
21(60.0 \%) \\
2(5.7 \%) \\
3(8.6 \%)\end{array}$ & $\begin{array}{c}2(13.3 \%) \\
2(13.3 \%) \\
8(53.3 \%) \\
3(20.0 \%) \\
0(0.0 \%)\end{array}$ & $\begin{array}{l}.849 \\
.929 \\
.662 \\
.123 \\
.242\end{array}$ \\
\hline BMI & $35.8(7.27)$ & $41.3(6.38)$ & $.013 *$ \\
\hline AHI & $22.9(18.99)$ & $36.1(33.05)$ & .080 \\
\hline CPAP Pressure & $9.7(2.75)$ & $10.4(3.31)$ & .489 \\
\hline
\end{tabular}

*Indicates statistical significance at the .05 level. 
the samples was married, having at least a high school education, and working full-time. While overall education status was similar for both groups, there was a statistically significant difference detected in Technical/Vocational education level $(\mathrm{p}=.010)$, with those not completing the study having a higher percentage of technical/vocational education $(26.7 \%)$ as compared to those completing the study $(2.9 \%)$.

\section{Compliant and noncompliant sample comparisons}

The sample of completed subjects ( $\mathrm{n}=35$ ) was stratified into CPAP compliant $(\geq 4$ hours/night on $70 \%$ of the observed nights) and CPAP noncompliant $(<4$ hours/night on $70 \%$ of the observed nights) groups for analysis. Table 6 outlines CPAP compliance for the completed study participants. CPAP compliance for those completing the study $(\mathrm{n}=35)$ was, on average, 313.6 minutes (5.2 hours) per night. The compliant group $(\mathrm{n}=21)$ at $\mathrm{T} 2$ (7 days post-CPAP) had a minimum, maximum, and mean use of 219.7 minutes ( 3.7 hours), 594.9 minutes ( 9.9 hours), and 396.3 minutes ( 6.6 hours) per night, respectively. Minimum CPAP use per night at $\mathrm{T} 2$ for the noncompliant group $(\mathrm{n}=14)$ was 38.0 minutes, with a maximum usage of 521.0 minutes ( 8.7 hours), and an average use of 273.6 minutes (4.6 hours). Compliance dropped for the noncompliant group at T3, with the minimum number of minutes used per night 0.0 , a maximum use of 267.6 (4.5 hours), and average use of 180.6 (3.0 hours). Compliance improved for the compliant group at T3, with minimum, maximum, and mean minutes of 271.0 (4.5 hours), 638.9 (10.6 hours), and 402.3 (6.7 hours). As noted in Table 7, when compliant subjects ( $n=21)$ were compared to the noncompliant $(n=14)$ completed subjects, there were no significant differences found on any of the demographic variables tested. 
Table 6

CPAP Compliance for Completed Subjects

\begin{tabular}{lccc}
\hline CPAP Compliance & $\begin{array}{c}\text { Minimum } \\
\text { Minutes/Night }\end{array}$ & $\begin{array}{c}\text { Maximum } \\
\text { Minutes/Night }\end{array}$ & $\begin{array}{c}\text { Average } \\
\text { Minutes/Night }\end{array}$ \\
\hline Total Group (n=35) & 0.00 & 638.90 & $313.59(138.69)$ \\
\hline
\end{tabular}

Time Point 2

$\begin{array}{lccc}\text { Compliant }(\mathbf{n = 2 1}) & 219.70 & 594.90 & 396.27(92.89) \\ \text { NonCompliant }(\mathbf{n}=\mathbf{1 4}) & 38.00 & 521.00 & 273.56(140.76)\end{array}$

Time Point 3

$\begin{array}{lccc}\text { Compliant }(\mathbf{n}=\mathbf{2 1}) & 271.00 & 638.90 & 402.26(90.03) \\ \text { NonCompliant }(\mathbf{n}=\mathbf{1 4}) & 0.00 & 267.60 & 180.59(78.02)\end{array}$


Table 7

Demographic Data Comparison of CPAP Compliant and Noncompliant Subjects

\begin{tabular}{|c|c|c|c|}
\hline Variable & $\begin{array}{c}\text { CPAP Compliant } \\
\text { Subjects }(n=21)\end{array}$ & $\begin{array}{l}\text { CPAP Noncompliant } \\
\text { Subjects }(n=14)\end{array}$ & p-value \\
\hline Age & $48.8(11.59)$ & $55.4(7.74)$ & .071 \\
\hline \multicolumn{4}{|l|}{ Gender } \\
\hline Male & $12(57.1 \%)$ & $8(57.1 \%)$ & 1.000 \\
\hline \multicolumn{4}{|l|}{ Ethnicity } \\
\hline Caucasian & $19(90.5 \%)$ & $12(85.7 \%)$ & .664 \\
\hline \multicolumn{4}{|l|}{ Education } \\
\hline$<12$ years & $0(0.0 \%)$ & $1(7.1 \%)$ & .214 \\
\hline High School & $9(42.9 \%)$ & $6(42.9 \%)$ & 1.000 \\
\hline Tech/Vocational & $0(0.0 \%)$ & $1(7.1 \%)$ & .214 \\
\hline College Grad (14yrs) & $5(23.8 \%)$ & $4(28.6 \%)$ & .752 \\
\hline College Grad (16yrs) & $5(23.8 \%)$ & $2(14.3 \%)$ & .490 \\
\hline College Grad (18yrs) & $2(9.5 \%)$ & $0(0.0 \%)$ & .234 \\
\hline \multicolumn{4}{|l|}{ Marital Status } \\
\hline Single & $4(19.0 \%)$ & $3(21.4 \%)$ & .863 \\
\hline Married & $12(57.1 \%)$ & $10(71.4 \%)$ & .392 \\
\hline Divorced & $3(14.3 \%)$ & $0(0.0 \%)$ & .139 \\
\hline Widowed & $1(4.8 \%)$ & $0(0.0 \%)$ & .407 \\
\hline Separated & $1(4.8 \%)$ & $1(7.2 \%)$ & .766 \\
\hline \multicolumn{4}{|l|}{ Work Status } \\
\hline Unemployed & $1(4.8 \%)$ & $3(21.4 \%)$ & .129 \\
\hline Part-time & $3(14.3 \%)$ & $2(14.3 \%)$ & 1.000 \\
\hline Full-time & $15(71.4 \%)$ & $6(42.9 \%)$ & .091 \\
\hline Disabled & $0(0.0 \%)$ & $2(14.3 \%)$ & .075 \\
\hline Retired & $2(9.5 \%)$ & $1(7.1 \%)$ & .805 \\
\hline BMI & $34.9(5.99)$ & $37.0(8.95)$ & .415 \\
\hline AHI & $22.2(19.49)$ & $24.0(18.87)$ & .782 \\
\hline CPAP Pressure & $10.1(2.79)$ & $9.2(2.67)$ & .334 \\
\hline
\end{tabular}

*Indicates statistical significance at the .05 level. 


\section{Repeated Measures Analysis of Variance}

Three separate repeated measures ANOVA tests (Table 8) were performed for each of the outcome variables. Measures of sleep activity, wake activity, and energy expenditure were entered into the ANOVA models to test for group effects (mean differences in each outcome variable between CPAP compliant and noncompliant groups), a time effect (mean differences in each outcome variable between time points) at baseline [T1], 7 days post-CPAP [T2], and 30 days post-CPAP [T3]), and a group $\mathrm{x}$ time interaction effect (mean differences among the compliant or noncompliant groups at any of the time points). As shown in Table 8, all three models demonstrated a significant group effect with all $\mathrm{p}$-values $<0.001$. Both sleep $(\mathrm{p}=.014)$ and wake $(\mathrm{p}=.024)$ demonstrated significant time effects; however, neither outcome had significant interaction effects $(p=.838$ and $p=.997$, respectively). Energy expenditure was not found to have a significant time effect $(p=.260)$ or interaction effect $(p=.878)$.

Figures 3, 4, and 5 graphically depict sleep activity (minutes), wake activity (minutes), and energy expenditure (kilocalories) over time, stratified by compliance, respectively. In Figure 3, sleep shows an increased sleep activity time in both compliant and noncompliant groups from T1-T2, and a continued increase from T1 to T3. Both groups had decreased wake activity time from T1-T2 with a continued decline from T1T3. While not statistically significant, Figure 5 shows a decrease in energy expenditure for both groups from $\mathrm{T} 1-\mathrm{T} 2$, with both the compliant and noncompliant groups showing a continued decrease from T1-T3. Although this research was interested in only measuring pre-CPAP (T1) to one week post-CPAP (T2) and one month post-CPAP (T3), the results for EE from repeated measures ANOVA (see Figure 5) showed an increase in EE from 
Table 8

Repeated Measures ANOVA of Sleep Activity, Wake Activity, and Energy Expenditure Over Time Stratified by Group

\begin{tabular}{|c|c|c|c|}
\hline Outcome & Predictors & $\mathbf{F}$ & p-value \\
\hline \multirow[t]{3}{*}{ Sleep Activity } & Compliance & 501.6 & $<.001 * * *$ \\
\hline & Time & 6.801 & $.014 * * *$ \\
\hline & Compliance*Time & 0.42 & .838 \\
\hline \multirow[t]{3}{*}{ Wake Activity } & Compliance & 1214.5 & $<.001 * * *$ \\
\hline & Time & 5.620 & $.024 * * *$ \\
\hline & Compliance*Time & 0.000 & .997 \\
\hline \multirow[t]{3}{*}{ Energy Expenditure } & Compliance & 647.3 & $<.001 * * *$ \\
\hline & Time & 1.312 & .260 \\
\hline & Compliance*Time & 0.024 & .878 \\
\hline
\end{tabular}


Figure 3. Sleep Activity Over Time Stratified by Compliance

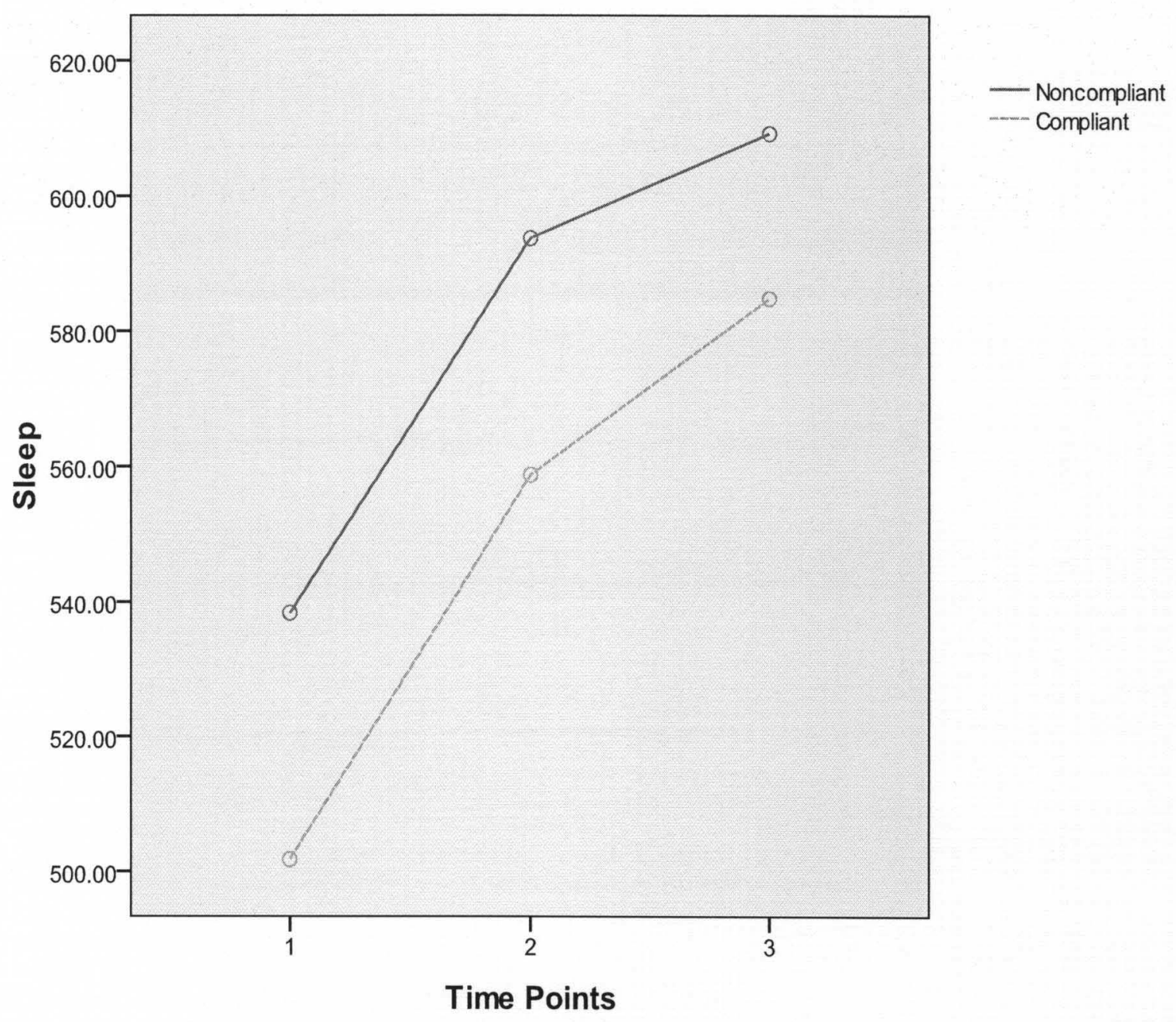


Figure 4. Wake Activity Over Time Stratified by Compliance

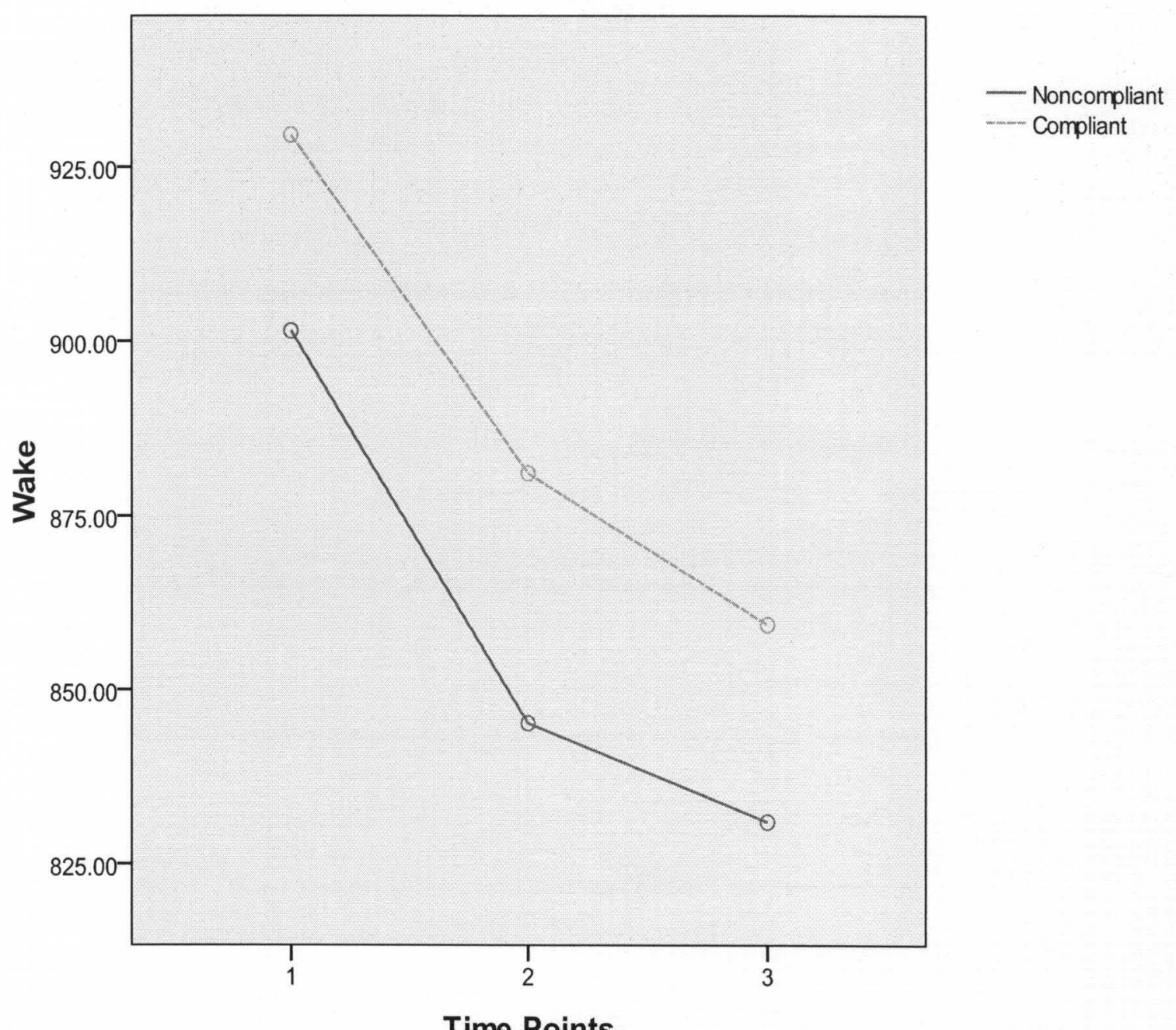


Figure 5. Energy Expenditure Over Time Stratified by Compliance

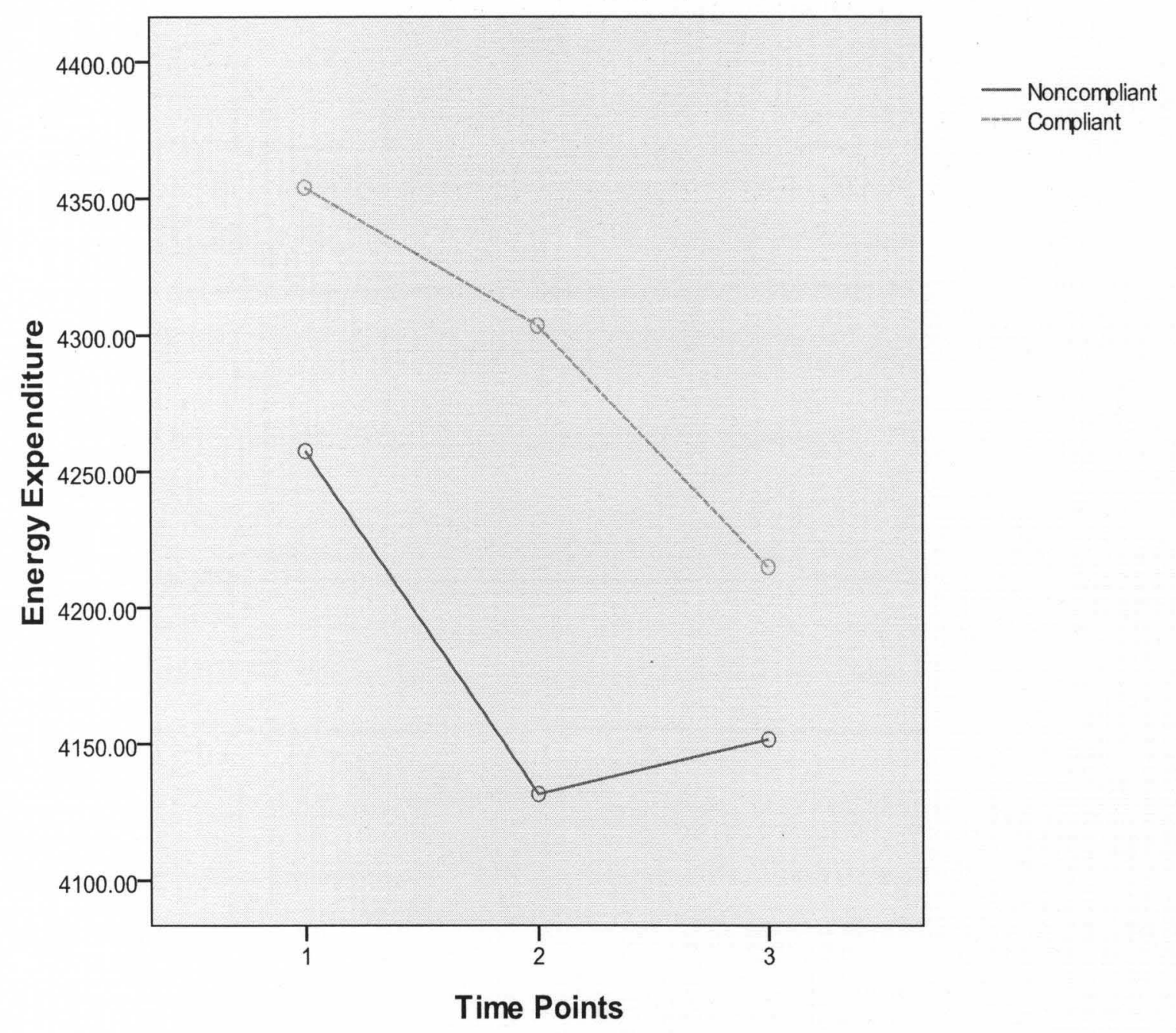


$\mathrm{T} 2$ to $\mathrm{T} 3$ in the CPAP noncompliant group.

\section{Paired $t$-tests}

Paired $t$-tests (Tables 9 and 10 ) were performed to provide more detailed information and allowed examination of mean differences in each variable from $\mathrm{T} 1$ to $\mathrm{T} 2$ $(n=38)$ and T1 to T3 $(n=35)$ for the entire group, and both compliant and noncompliant groups. Sleep time increased from pre-CPAP (baseline) to 7 days post-CPAP (one week) and 30 days post-CPAP (one month) regardless of compliance status. Statistically significant differences in sleep were demonstrated in the total group $(p=.004)$ and compliant group $(\mathrm{p}=.012)$ from T1-T2, and the total group $(\mathrm{p}=.010)$ and noncompliant group $(\mathrm{p}=.007)$ from $\mathrm{T} 1-\mathrm{T} 3$. Wake time showed a decrease across the same time points for all groups, with significant decreases for the total group $(\mathrm{p}=.009)$ and compliant group $(\mathrm{p}=.031)$ from $\mathrm{T} 1-\mathrm{T} 2$, and the total group $(\mathrm{p}=.019)$ and noncompliant group $(\mathrm{p}=.008)$ from T1-T3. Energy expenditure revealed decreases in all groups, across both time points, although not significant. While an increase in energy expenditure from T2 to T3 can be seen in Figure 5 for the noncompliant group, additional analyses (Table 11) found this change was not statistically significant $(\mathrm{p}=.315)$.

Consistent with repeated measures ANOVA testing, there were total group differences noted for sleep activity and wake activity from T1-T2 and T1-T3; however, energy expenditure did not reveal any significant differences for the total group between any time points. Interestingly, mean differences in sleep and wake were significant in the compliant group from T1-T2, but were not from T1-T3. Conversely, significant differences in sleep and wake were observed in the noncompliant group from T1-T3, but not from T1-T2. Likewise, repeated measures ANOVA demonstrated significant group 
Table 9

Paired t-tests: Sleep Activity, Wake Activity, and Energy Expenditure Stratified by Group at Adjacent Time Points 1 to 2

\begin{tabular}{lcccc}
\hline Outcome Variable & $\begin{array}{c}\text { Baseline } \\
\text { Pre-CPAP }\end{array}$ & $\begin{array}{c}\text { One Week } \\
\text { Post-CPAP }\end{array}$ & $\begin{array}{c}\text { Mean } \\
\text { Difference }\end{array}$ & $\begin{array}{c}\text { p- } \\
\text { value }\end{array}$ \\
& T1 & T2 & T1-T2 & \\
\hline Sleep Activity & & Minutes(SD) & & \\
Total group (n=38) & $526.4(115.8)$ & $585.4(27.5)$ & $59.0(116.9)$ & $.004^{*}$ \\
Compliant (n=27) & $532.2(116.3)$ & $596.8(169.7)$ & $64.6(124.9)$ & $.012^{*}$ \\
Noncompliant (n=11) & $512.1(118.8)$ & $557.4(173.4)$ & $45.2(98.7)$ & .159 \\
& & & & \\
\hline Wake Activity & & & & \\
Total group (n=38) & $908.9(117.0)$ & $854.2(169.7)$ & $-54.7(123.2)$ & $.009^{*}$ \\
Compliant (n=27) & $901.1(117.6)$ & $842.6(170.1)$ & $-58.6(133.4)$ & $.031^{*}$ \\
Noncompliant (n=11) & $927.8(118.8)$ & $\mathbf{8 8 2 . 6 ( 1 7 3 . 4 )}$ & $-45.2(98.8)$ & .160
\end{tabular}

\begin{tabular}{lrrrrr}
\hline Energy Expenditure & \multicolumn{5}{c}{ Kilocalories(SD) } \\
Total group (n=38) & $4285.8(908.3)$ & $4183.6(982.3)$ & $-102.3(527.0)$ & .239 \\
Compliant $(\mathbf{n}=\mathbf{2 7})$ & $4265.0(856.7)$ & $4132.9(985.6)$ & $-132.1(554.6)$ & .227 \\
Noncompliant $(\mathbf{n}=\mathbf{1 1})$ & $4336.8(1067.7)$ & $4307.7(1010.4)$ & $-29.1(468.6)$ & .841
\end{tabular}

*Indicates statistical significance at the .05 level. 
Table 10

Paired t-tests: Sleep Activity, Wake Activity, and Energy Expenditure Stratified by Group at Distant Time Points 1 to 3

\begin{tabular}{|c|c|c|c|c|}
\hline Outcome Variable & $\begin{array}{c}\text { Baseline } \\
\text { Pre-CPAP } \\
\text { T1 }\end{array}$ & $\begin{array}{l}\text { One Month Post } \\
\text { CPAP } \\
\text { T3 }\end{array}$ & $\begin{array}{c}\text { Mean } \\
\text { Difference } \\
\text { T1-T3 }\end{array}$ & $\begin{array}{c}\text { p- } \\
\text { value }\end{array}$ \\
\hline Sleep Activity & & Minutes(SD) & & \\
\hline Total group $(n=35)$ & $516.4(112.9)$ & $594.6(201.5)$ & $78.1(168.5)$ & $.010^{*}$ \\
\hline Compliant $(\mathrm{n}=\mathbf{2 1})$ & $501.8(96.7)$ & $584.8(230.7)$ & $82.9(209.0)$ & .084 \\
\hline Noncompliant $(n=14)$ & $538.4(134.5)$ & $609.2(154.7)$ & $70.9(83.6)$ & $.007^{*}$ \\
\hline Wake Activity & & Minutes(SD) & & \\
\hline Total group $(n=35)$ & $918.4(114.7)$ & $848.0(199.9)$ & $-70.4(169.6)$ & $.019^{*}$ \\
\hline Compliant $(\mathrm{n}=21)$ & $929.6(101.4)$ & $859.4(227.9)$ & $-70.3(210.6)$ & .142 \\
\hline Noncompliant $(n=14)$ & $901.5(134.5)$ & $831.0(154.9)$ & $-70.5(83.8)$ & $.008^{*}$ \\
\hline Energy Expenditure & & Kilocalories(SD) & & \\
\hline Total group $(n=35)$ & $4315.5(937.3)$ & $4189.6(1042.0)$ & $-125.9(611.3)$ & .231 \\
\hline Compliant $(n=21)$ & $4354.1(794.6)$ & $4214.9(1014.3)$ & $-139.2(704.2)$ & .376 \\
\hline Noncompliant $(n=14)$ & $4257.6(1149.0)$ & $4151.6(1119.9)$ & $-105.9(462.4)$ & .407 \\
\hline
\end{tabular}

*Indicates statistical significance at the .05 level. 
Table 11

Paired t-tests: Sleep Activity, Wake Activity, and Energy Expenditure Stratified by Group at Time Points 2 to 3

\begin{tabular}{|c|c|c|c|c|}
\hline Outcome Variable & $\begin{array}{c}\text { One Month } \\
\text { Post-CPAP } \\
\text { T2 }\end{array}$ & $\begin{array}{l}\text { One Month Post } \\
\text { CPAP } \\
\text { T3 }\end{array}$ & $\begin{array}{c}\text { Mean } \\
\text { Difference } \\
\text { T2-T3 }\end{array}$ & $\begin{array}{c}\text { p- } \\
\text { value }\end{array}$ \\
\hline Sleep Activity & & Minutes(SD) & & \\
\hline Total group $(n=35)$ & $572.8(161.1)$ & $594.6(201.5)$ & $21.8(84.2)$ & .135 \\
\hline Compliant $(n=21)$ & $558.8(170.9)$ & $584.8(230.7)$ & $26.0(94.3)$ & .220 \\
\hline Noncompliant $(n=14)$ & $593.8(148.8)$ & $609.2(154.7)$ & $15.5(69.2)$ & .418 \\
\hline Wake Activity & & Minutes(SD) & & \\
\hline Total group $(n=35)$ & $866.7(161.5)$ & $848.0(199.9)$ & $-18.7(80.8)$ & .180 \\
\hline Compliant $(n=21)$ & $881.1(171.1)$ & $859.4(227.9)$ & $-21.8(89.5)$ & .279 \\
\hline Noncompliant $(n=14)$ & $845.2(149.6)$ & $831.0(154.9)$ & $-14.2(68.8)$ & .454 \\
\hline Energy Expenditure & & Kilocalories(SD) & & \\
\hline Total group $(n=35)$ & $4234.8(1007.7)$ & $4189.6(1042.0)$ & $-45.2(335.2)$ & .430 \\
\hline Compliant $(n=21)$ & $44303.5(1000.7)$ & $4214.9(1014.3)$ & $-88.6(394.3)$ & .315 \\
\hline Noncompliant $(n=14)$ & $4131.7(1046.9)$ & $4151.6(1119.9)$ & $-19.9(216.8)$ & .737 \\
\hline
\end{tabular}

${ }^{*}$ Indicates statistical significance at the .05 level. 
effect in energy expenditure, but paired $t$-tests failed to reveal statistically significant mean differences for the total group, compliant group, or noncompliant group. While these findings seem counterintuitive, they are consistent with Simpson's Paradox, in which a correlation or trend is observed when groups are stratified to test for marginal effects, but becomes non-significant when groups are combined (Cohen, 1988). This is often a result of confounding factors and caution should be exercised in making causal inferences about the data (Cohen), specifically the effect of CPAP on sleep activity, wake activity, and energy expenditure. 


\title{
CHAPTER V
}

\section{DISCUSSION}

\author{
Introduction
}

The purpose of this research was to examine the influence of CPAP on sleep activity, wake activity, and energy expenditure. Overweight (BMI $\geq 25)$ and obese (BMI $>30$ ) adult patients with $\mathrm{OSA}(\mathrm{AHI} \geq 5$ ) were the targeted population for this study, as their risk of comorbidities are greater than those non-overweight/obese OSA patients. Sleep activity, wake activity and energy expenditure were measured prior and subsequent to initiating CPAP therapy, testing for differences that exist before and after therapy over time. A conceptual model (Figure 1, p. 5) was developed to provide the framework for assessing overweight/obesity (BMI), OSA (polysomnography-AHI), sleep activity (actigraphy), wake activity (actigraphy), energy expenditure (actigraphy) and CPAP compliance (internal compliance meter). The propositions linking these variables included the relationships between overweight/obesity and OSA, how sleep fragmentation/disruption and daytime fatigue/sleepiness are associated with OSA, and how these concepts link OSA to increased sleep activity and decreased wake activity, with a decrease in 24-hour total energy expenditure. The model further included CPAP treatment affecting sleep consolidation (less sleep fragmentation/disruption), improving wakefulness (less daytime fatigue/sleepiness), and how (respectively) these concepts negatively correlate CPAP treatment (and level of compliance) with sleep activity, and 
positively correlate CPAP therapy with wake activity and 24-hour total energy expenditure.

\section{Findings}

As stated in the hypotheses, and following the conceptual framework, the net loss of total 24-hour sleep activity and gain in total 24-hour wake activity was expected to result in an overall increase in total 24-hour energy expenditure following CPAP treatment; however, the statistical analyses demonstrated the following findings relative to the 3 hypothesized study outcomes.

\section{Hypothesis 1}

The first study hypothesis stated that subjects would have a decrease in sleep activity as a result of using CPAP, with decreases in 24-hour total sleep activity, both 7 days and 30 days post CPAP, as compared to pre-CPAP. The results actually showed the following:

H1) Subjects had a statistically significant increase in sleep activity as a result of using CPAP (measured by the Actiwatch $($ )

H1a. compared to pre-CPAP, there was a statistically significant increase in 24-hour total sleep activity (time in minutes) 7 days post-CPAP

H1b. compared to pre-CPAP, there was a statistically significant increase in 24-hour total sleep activity (time in minutes) 30 days post-CPAP

The results demonstrated statistically significant increases in total 24-hour sleep activity, in minutes, from the pre-CPAP time point (baseline) to both the one week and 30 day post-CPAP time points. Contrary to what was hypothesized, the subjects in this study showed a statistically significant increase in their 24-hour sleep activity, with 
statistically significant differences between the CPAP compliant and noncompliant groups. When paired t-tests were performed from $\mathrm{T} 1$ to $\mathrm{T} 2$, the total group $(\mathrm{n}=38)$ and the CPAP compliant group $(\mathrm{n}=27)$ had statistically significant mean differences in sleep activity. While from T1-T3, it was the total group $(n=35)$ and noncompliant group $(n=14)$ who had statistically significant differences in mean sleep activity.

At baseline, all subjects were exceeding 8 hours ( $>480$ minutes) of sleep activity time over 24 hours (see Tables 9 and 10), with a minimum mean sleep time of 501.8 minutes. They continued to exceed 8 hours and extend their 24 hour total sleep activity time following CPAP treatment at the seven day and 30 day time points. A graph (Figure 3, p. 84) generated from repeated measures ANOVA further illustrates this point and shows group differences, with noncompliant subjects having more sleep activity time at each of the three time points as compared to the compliant subjects. There are no empirical studies to either support or negate these findings, only anecdotal references and clinical observations to offer mixed thoughts about the effect of using CPAP on sleep time. Clinically, patients report sleeping fewer hours and feeling more refreshed, so it is difficult to determine if these study subjects were sleeping longer as a consolidated sleep period at night or had more total sleep activity time because they were more sedentary or napping during the day, at any of the measured time points. This is a limitation of using actigraphy alone to measure ambulatory sleep activity for this study; however, this device has demonstrated reliability in distinguishing sleep and wake when compared to electroencepaholgraphic measures with polysomonography, and the results can be interpreted with a fairly high degree of confidence (Ancoli-Israel, 2005; Kushida et al., 
2001; Babin, Lee, Halko, Boudreau \& George, 1997; Blood, Sack, Percy \& Pen, 1997;

Oakely, 1997; Sadeh, Hauri, Kripke \& Lavie, 1995).

\section{Hypothesis 2}

The second study hypothesis stated that subjects would have an increase in wake activity as a result of using CPAP, with increases in 24-hour total wake activity, both 7 days and 30 days post $\mathrm{CPAP}$, as compared to pre-CPAP. The results actually showed the following:

H2) Subjects had a statistically significant decrease in wake activity as a result of using CPAP (measured by the Actiwatch ${ }^{\circledR}$ )

$\mathrm{H} 2 \mathrm{a}$. compared to pre-CPAP, there was a statistically significant decrease in 24-hour total wake activity (time in minutes) 7 days post-CPAP $\mathrm{H} 2 \mathrm{~b}$. compared to pre-CPAP, there was a statistically significant decrease in 24-hour total wake activity (time in minutes) 30 days post-CPAP Again, contrary to what was hypothesized, the subjects in this study showed a statistically significant decrease in their 24 hour wake activity. Results of repeated measures ANOVA detected statistically significant group (compliant and noncompliant) and time differences in total 24 hour wake activity (in minutes) from the pre-CPAP time point (baseline) to both the one week and 30 day post-CPAP time points without significant interaction between groups or time points. Because there were only two groups, post-hoc testing was not conducted. Likewise, because there was no evidence of statistically significant interaction between groups and time, further data analyses was not performed. Interestingly, paired $t$-tests revealed the total group $(\mathrm{n}=38)$ from $\mathrm{T} 1$ to $\mathrm{T} 2$ and compliant group $(\mathrm{n}=27)$ to have statistically different mean wake activity times, but from 
T1 to T3 the total group $(n=35)$ and noncompliant group $(n=14)$ showed statistically different wake activity time means. From baseline through T2, the compliant subjects had more mean wake activity time (and less mean sleep activity time) than the noncompliant subjects, which was just the opposite from baseline to T3. This finding is not fully understood and cannot be explained by differences in the demographic data collected, as there were no statistically significant differences between groups. Further exploration of this should be considered in future studies.

\section{Hypothesis 3}

The third study hypothesis stated that subjects would have an increase in energy expenditure as a result of using CPAP, with increases in 24-hour total energy expenditure, both 7 days and 30 days post CPAP, as compared to pre-CPAP. The results actually showed the following:

H3) Subjects had a decrease (not statistically significant) in energy expenditure subsequent to initiating CPAP therapy (measured by the Actical ${ }^{\circledR}$ )

H3a. compared to pre-CPAP, there was a decrease (not statistically significant) in 24-hour total energy (kilocalorie) expenditure 7 days postCPAP

H3b. compared to pre-CPAP, there was a decrease (not statistically significant) in 24-hour total energy (kilocalorie) expenditure 30 days postCPAP

Energy expenditure was not found to have a statistically significant time effect; therefore the study hypothesis for this outcome was not supported; however, repeated measures ANOVA detected statistically significant group differences among the CPAP 
compliant and noncompliant (see Table 8). Paired $t$-tests were performed (Tables 9 and 10 ), but were unable to confirm any statistically significant differences in EE in the total, compliant, and noncompliant groups, across any time point. Number of calories burned decreased, rather than increased, for both the CPAP compliant and noncompliant groups from $\mathrm{T} 1$ to $\mathrm{T} 2$ and $\mathrm{T} 1$ to $\mathrm{T} 3$, with the compliant group expending more energy than the noncompliant group at all time points.

\section{Conclusions}

These study findings were the opposite of what was hypothesized, with increased total 24-hour sleep activity time, decreased total 24 -hour wake activity time, and decreased total 24-hour energy expenditure, regardless of CPAP treatment or level of compliance. The groups had statistically significant mean differences in sleep activity and wake activity from pre-CPAP to post-CPAP at one week and one month; however the direction of change was consistent for both groups, with sleep activity increasing and wake activity decreasing. At baseline, and continuing through T2 and T3, the CPAP compliant group showed less sleep activity, more wake activity, and more calories burned than the noncompliant group. When assessing demographics of the compliant versus noncompliant groups, there were no significant differences in any of the variables tested to account for this finding.

CPAP compliance was an important variable in this study and has a wide degree of variance in the literature, with ranges from 46 to $80 \%$ (Basner, 2007; Grunstein, 2005; Kribbs et al., 1993; Weaver et al., 1997). In this study, approximately $71 \%$ were compliant after the first week of home use, and approximately $60 \%$ were compliant after one month of home use. While not expected, subjects in this study had greater sleep 
activity, less wake activity, and expended less energy after using CPAP, whether they were compliant or not. Empiric evidence has shown CPAP to decrease the number of arousals and awakenings during sleep due to apneic events; however, this did not translate to more consolidated/less total 24 hour sleep or greater 24 hour wake time/more physical activity for this group of study participants. Furthermore, these findings showed fewer calories burned post-CPAP, but differences in energy expenditure were not statistically significant.

Unfortunately there is no direct link to or succinct explanation for this study's findings; however, two relatively recent publications by West, Kohler, Nicoll, \& Stradling (2009) and Otake et al. (2010) studied the effect of CPAP on sleep/wake activity using actigraphy. West et al. studied 36 men with OSA and found there were no significant differences in activity levels one week before the initiation of CPAP as compared to one week after CPAP therapy. Otake et al. confirmed agreement between PSG and actigraphy data and determined subjects treated after one month of CPAP had significantly less activity levels/movement than prior to CPAP. Both the effects of OSA and CPAP therapy on sleep/wake activity and energy expenditure remain unclear and lack sufficient empirical evidence to support the current study results.

This study has shown that OSA subjects using CPAP have more sleep activity than prior to treatment, subsequently reducing 24-hour total wake activity time. Less wake time, combined with fewer arousals and awakenings due to apnea, could feasibly reduce total caloric expenditure. Although there was not a statistically significant decrease in daily energy expenditure following CPAP use, the decline in calories burned following CPAP treatment may be a longer term factor in weight gain for OSA patients. 


\section{Implications}

This research was adequately powered to demonstrate that CPAP use is a statistically significant factor affecting sleep activity time, wake activity time, and energy expenditure. Regardless of CPAP compliance (nightly hours) and length of use (one week or one month), the entire post-CPAP group demonstrated more sleep activity, had less wake activity, and burned fewer calories. This would potentially translate to an increase in weight over time. From a clinical perspective, the results of this study do not support the use of CPAP as a potential weight loss measure in overweight and obese OSA patients, and emphasize the need for the inclusion of behavioral weight management and weight loss strategies in an at-risk population for comorbid illnesses. The extended use of actigraphy in both the research and practice setting would be a beneficial adjunct to monitoring sleep/wake activity and energy expenditure and set behavioral goals and outcomes. Studies have shown that those who self-report sleeping fewer hours ( $\leq 6$ hours/day) tend to gain weight (Hayes, Xu, Babineau, \& Patel, 2011; ) and have higher incidences of circulating leptin (adipose-derived hormone) and adipokines (immunomodulating cytokines secreted by adipose tissue) levels (Hayes, et al.). These high levels have been linked to greater risk of increased inflammatory markers contributing to cardiovascular and cerebrovascular disease, insulin resistance, and diabetes (Leinum, Dopp, \& Morgan, 2009; Vgontzas, Zoumakis, \& Bixler, 2004); Patel, Zhu, \& Storfer-Isser, 2009).

The current study showed those who completed the study $(n=35)$ were older and had a lower BMI than those who did not complete the study $(n=15)$. These were the only statistically significant differences among demographics. The outcomes of this study 
may have been quite different had the $30 \%$ early-term subjects completed the study. This is also an important consideration for recruitment and retention for future research. Of the entire completed sample $(n=35)$, there were no statistically significant differences in demographic findings between those who were compliant with CPAP and those who were not. Interestingly, those who completed all 3 time points averaged more than 8 hours of sleep both before and after CPAP treatment. This could be a result of actigraphy recording sleep during waking sedentary activity. Possibly, previous research utilizing subjective sleep report may underestimate true sleep time. Again, this may support the use of actigraphy in combination with subject sleep/wake diaries in both research and practice.

\section{Limitations}

While those patients who are unable to speak, read, write and understand the English language were excluded from study participation, this did not pose a significant restriction to recruitment, as the practice setting this sample was drawn from does not typically have a high percentage of non-English speaking patients. However, external validity and generalizability was limited for several reasons. Choosing only one practice setting had the disadvantage of not having a more heterogeneous group, and including only one group did not allow for a control group or group comparisons, other than compliance. For this study, the inclusion of several sites and patient groups was cost prohibitive and not feasible without additional research staff. Conversely, an advantage to a single site and group was efficiency, consistency and better control of patient care and follow-up, diagnostic testing, scoring of data, and titration of CPAP. 
To reduce threats to internal validity, acknowledgement and control of as many identified confounding variables as possible was exercised; however, the apparent Simpson's Paradox observed during data analyses revealed the likely influence of such variables. Some of these variables were anticipated and accounted for in the inclusion/exclusion criteria and CPAP compliance data. The use of subjective sleep diaries, and activity and food diaries (during the days of Actiwatch ${ }^{\circledR}$ and Actical ${ }^{\circledR}$ use) would have offered further support and/or explanation of data and should be considered in future studies. Additionally, ANOVA assumes subjects will be randomly assigned to either a control or treatment group, so that two groups do not have any systematic difference except for the treatment applied. This study design involved non-random assignment; however, in the case of self-selection, the repeated measures treatment effect model was expected to produce reliable estimates of the mean differences.

The study was further limited by the possibility that pre- and post-CPAP differences in sleep/wake activity and energy expenditure may be detected as a result of subject response to study participation and knowledge of what is being measured rather than real change (Munro, 2005). A repeated measures study design was expected to provide some control against such contrived patient activity, or even variation in activity levels related to individual and situational factors at the time of measurement. An extension of this longitudinal study may have offered more robust findings.

\section{Recommendations}

Research in the area of sleep/wake and energy expenditure in overweight and obese OSA patients has become increasingly important and clinically relevant in a population where the incidence of OSA, weight gain, and resulting comorbid illness 
continues to rise (Leinum, Dopp, \& Morgan, 2009). There is an abundance of evidence linking OSA, obesity, cardiovascular disease, metabolic risk, and the benefit of CPAP therapy; however, there is limited data regarding the effect CPAP treatment has on sleep, wake, and energy expenditure in an ambulatory and long-term setting. Although the results of this study failed to support the conceptual framework developed to guide the study hypotheses, this study offers a substantial review of past and currently available literature and provides a foundation for future clinical investigation. The current conceptual framework and hypotheses were developed from the existing body of knowledge in this field which was heavily weighted on anecdotal studies and clinical observation, further highlighting the need for greater empirical testing. Additional research in overweight and obese patients with OSA is needed, and should be pursued with model revision of sleep/wake and energy expenditure concepts that link OSA with obesity, cardiovascular disease and metabolic dysfunction as a framework for larger prospective studies. 


\section{REFERENCES}

Ainsworth, B. E., Haskell, W. L., Leon, A. S., Jacobs, D. R., Montoye, H. J., Sallis, J. F., et al. (1993). Compendium of physical activities: Classification of energy costs of human physical activities. Medicine and Science in Sports and Exercise, 25(1), $71-80$.

Albert-Tulkens, G., Culee, C., \& Rijckevorsel, K. H., et al. (1987). Ambulatory evaluation of sleep disturbance and therapeutic effects in sleep apnea syndrome by wrist activity monitoring. American Review of Respiratory Disease, 136, 851856.

Al-Delaimy, W. K., Manson, J. E., Willett, W. C., Stampfer, M. J., \& Hu, F. B. (2002). Snoring as a risk factor for type II diabetes mellitus: A prospective study. American Journal of Epidemiology, 155(5), 387-393.

Allison, D. B., Fontaine, K. R., \& Manson, J. E., et al. (1999). Annual deaths attributable to obesity in the United States. $J A M A, 282,1530-1538$.

American Academy of Sleep Medicine Task Force (1999). Sleep-related breathing disorders in adults: Recommendations for syndrome definition and measurement techniques in clinical research. Sleep, 22, 667-689.

American Thoracic Society, Medical Section of The American Lung Association (1989). Indications and standards for cardiopulmonary sleep studies. American Review of Respiratory Disease, 139, 559-568.

Ancoli-Israel, S. (2005). Actigraphy. In M.H. Kryger, T. Roth \& W.C. Dement (Eds.), Principles and Practice of Sleep Medicine (4th ed., pp. 1459-1467). Philadelphia: Elsevier Saunders.

Ancoli-Israel, S., Gehrman, P., \& Kripke, D., et al. (2001). Long-term follow-up of sleep disordered breathing in older adults. Sleep Medicine, 2(6), 511-516.

Andreas, S., Schulz, R., Werner, G. S., \& Kreuzer, H. (1996). Prevalence of obstructive sleep apnoea in patients with coronary artery disease. Coronary Artery Disease, $7(7), 541-545$.

Ayas, N. T., Patel, S. R., \& Malhotra, A., et al. (2004). Auto-titrating versus standard continuous positive airway pressure for the treatment of obstructive sleep apnea: Results of a meta-analysis. Sleep, 27, 249-253. 
Babin, L., Lee, S., Haldo, s., Boudreau, A.C. \& George, C.F.P. (1997). Determining sleep-wake activity using Actiwatch ${ }^{\circledR}$. Sleep Research, 26, 640.

Babu, A. R., Herdegen, J., Fogelfeld, L., Shott, S., \& Mazzone, T. (2005). Type 2 diabetes, glycemic control, and continuous positive airway pressure. Archives of Internal Medicine, 165(4), 447-452.

Bady, E., Achkar, A., Pascal, S., Orvoen-Frija, E., \& Laaban , J. P. (2000). Pulmonary arterial hypertension in patients with sleep apnoea syndrome. Thorax, 55(11), 934-939.

Baguet, J. P., Hammer, L., \& Levy, P., et al. (2005). The severity of oxygen desaturation is predictive of carotid wall thickening and plaque occurrence. Chest, 128, 34073412.

Baldwin, C. M., Griffith, K. A., \& Nieto, F. J. (2001). The association of sleep-disordered breathing and sleep symptoms with quality of life in the Sleep Heart Health Study. Sleep, 24(96-105).

Bardwell, W. A., Ancoli-Israel, S., Berry, C. C., \& Dimsdale, J. (2001). Neuropsychological effects of one-week continuous positive airway pressure treatment in patients with obstructive sleep apnea: A placebo-controlled study. Psychosomatic Medicine, 63, 579-584.

Basner, R.C. (2007). Continuous positive airway pressure for obstructive sleep apnea. New England Journal of Medicine, 356, 1751-1758.

Bassetti, C., \& Aldrich, M. S. (1999). Sleep apnea in acute cerebrovascular diseases: Final report on 128 patients. Sleep, 22(2), 217-223.

Bassetti, C., Aldrich, M. S., Chervin, R. D., \& Quint, D. (1996). Sleep apnea in patients with transient ischemic attack and stroke: A prospective study of 59 patients. Neurology, 47(5), 1167-1173.

Becker, H. F., Jerrentrup, A., \& Ploch, T., et al. (2003). Effect of nasal continuous positive airway pressure treatment in patients with obstructive sleep apnea. Circulation, 107, 68-73.

Bedard, M. A., Montplaisir, J., \& Richer, F., et al. (1991). Obstructive sleep apnea syndrome: Pathogenesis of neuropsychological deficits. Journal of Clinical and Experimental Neuropsychology, 13(6), 950-964.

Bixler, E. O., Vgontzas, A. N., \& Lin, H. M., et al. (2001). Prevalence of sleepdisordered breathing in women: Effects of gender. American Journal of Respiratory and Critical Care Medicine, 163, 608-613. 
Blood, M., Sack, R., Percy, D. \& Pen, J. (1997). Determination of sleep: A comparison of sleep detection by wrist actigraphy, behavioral response, and polysomnography. Sleep, 20(6), 388-395.

Borak, J., Cieslicki, J. K., \& Koziej, M., et al. (1996). Effects of CPAP treatment on psychological status in patients with severe obstructive sleep apnoea. Journal of Sleep Research, 5(2), 123-127.

Buchwald, H., Avidor, Y., \& Braunwald, E., et al. (2004). Bariatric surgery: A systematic review and meta-analysis. $J A M A, 292,1724-1737$.

Caples, S.M., Gami, A.S., Somers, V.K. (2005). Obstructive sleep apnea. Annals of Internal Medicine, 142, 187-197.

Carskadon, M. A., \& Dement, W. C. (2005). Normal human sleep: An overview. In Kryger, M.H, Roth, T., Dement, W.C. (Eds.), Principles and practice of sleep medicine (4th ed., pp. 13-23). Philadelphia: Elsevier Saunders.

Caterson, I. D., Hubbard, V., Bray, G. A., Grunstein, R., Hansen, B. C., Hong, Y., et al. (2004). Prevention conference VII: Obesity, a worldwide epidemic related to heart disease and stroke: Group III: Worldwide comorbidities of obesity. Circulation, 110, 476-483.

Chaput, J. P., Lord, C., Aubertin-Leheudre, M., Dionne, I. J., Khalil, A., \& Tremblay, A. (2007). Is overweight/obesity associated with short sleep duration in older women? Aging Clinical and Experimental Research, 19(4), 290-294.

Chesson, A. L., Berry, R. B., \& Pack, A. (2003). Practice parameters for the use of portable monitoring devices in the investigation of suspected obstructive sleep apnea in adults. Sleep, 26(7), 907-913.

Chin, K., Shimizu, K., \& Nakamura, T., et al. (1999). Changes in intra-abdominal visceral fat and serum leptin levels in patients with obstructive sleep apnea syndrome following nasal continuous positive airway pressure therapy. Circulation, 100, 706-712.

Cohen, J. (1988). Statistical power analysis for the behavioral sciences. Hillsdale, NJ: Lawrence Erlbaum Associates.

Coughlin, S. R., Mawdsley, L., Mugarza, J. A., \& Calverley, P. M. Wilding, J.P. (2004). Obstructive sleep apnoea is independently associated with an increased prevalence of metabolic syndrome. European Heart Journal, 25(9), 735-741.

Crouter, S.E., Churilla, J.R. \& Bassett, D.R. (2006). Estimating energy expenditure using accelerometers. European Journal of Applied Physiology, 98(6), 601-612. 
Dement, W. C. (2005). History of sleep medicine. Neurologic Clinics, 23, 945-965.

Dempsey, J. A., Veasey, S. C., Morgan, B. J., \& O’Donnell, C. P. (2010). Pathophysiology of obstructive sleep apnea. Physiological Reviews, 90, 47-112.

Derderian, S. S., Bridenbaugh, R. H., \& Rajagopal, K. R. (1998). Neuropsychologic symptoms in obstructive sleep apnea improve after treatment with nasal continuous positive airway pressure. Chest, 94(5), 2100-2105.

Doherty, L. S., Kiely, J. L., Swan, V., \& McNicholas, W. T. (2005). Long-term effects of nasal continuous positive airway pressure therapy on cardiovascular outcomes in sleep apnea syndrome. Chest, 127(6), 2076-2084.

Drager, L. F., Bortolotto, L. A., \& Lorenzi, M. C., et al. (2005). Early signs of atherosclerosis in obstructive sleep apnea. American Journal of Respiratory and Critical Care Medicine, 172, 613-618.

Dyken, M. E., Ali, M., Raman, S., \& Eppen, K. E. (2007). Obesity. In C.A. Kushida (Ed.), Obstructive sleep apnea: Pathophysiology, comorbidities and consequences (pp. 355-376). New York: Informa Healthcare USA, Inc.

Elbaz, M., Roue, G. M., Lofaso, F., \& Quera, S. M. (2002). Utility of actigraphy in the diagnosis of obstructive sleep apnea. Sleep, 25, 527-531.

Engleman, H. M., Kingshott, R. N., \& Martin, S. E., et al. (2000). Cognitive function in the sleep apnea/hypopnea syndrome. Sleep, 23, S102-S108.

Faul, F., Erdfelder, E., Lang, A-G, \& Buchner, A. (2007). G*Power 3: A flexible statistical power analysis program for the social, behavioral, and biomedical sciences. Behavior Research Methods, 39, 175-191.

Feeney, A., Pfeiffer, K., Hutto, B., McIver, K., Blair, S., Vena, J., LaMonte, M. \& Hooker, S. (2007). Obesity, 15, A111.

Findley, L. J., \& Suratt, P. M. (2001). Serious motor vehicle crashes: the cost of untreated sleep apnoea. Thorax, 56(7), 505.

Flegal, K. M., Graubard, B. I., Williamson, D. F., \& Gail, M. H. (2005). Excess deaths associated with underweight, overweight and obesity. JAMA, 293, 1861-1867.

Gadnoadoux, F., Nguyen, X. L., Rakotonanahary, D., Vidal, S., \& Fleury, B. (2004). Wrist actigraphic estimation of sleep time under nCPAP treatment in sleep apnea patients. European Respiratory Journal, 23, 891-895. 
Gangwisch, J. E., Malaspina, D., Boden-Albala, B., \& Heymsgield, S. B. (2005). Inadequate sleep as a risk factor for obesity: Analysis of the NHANES I. Sleep, $28,1289-1296$.

Gonsalves, M. A., Paiva, T., Ramos, E., \& Guilleminault, C. (2004). Obstructive sleep apnea syndrome, sleepiness and quality of life. Chest, 125, 2091-2096.

Grunstein, R. (2005). Continuous positive airway pressure treatment for obstructive sleep apnea-hypopnea syndrome. In M.H. Kryger, T. Roth, W.C. Dement (Ed.), Principles and practice of sleep medicine (4th ed., pp. 1066-1080). Philadelphia: Elsevier Saunders.

Grunstein, R. R, Stenloff, K., Hedner, J. A., Peltonen, M., Karason, K., and Sjostrom, L. (2007). Two year reduction in sleep apnea symptoms and associated diabetes incidence after weight loss in severe obesity. Sleep, 30(6), 703-710.

Guilleminault, C., \& Bassiri, A. (2005). Clinical features and evaluation of obstructive sleep apnea-hypopnea syndrome and upper airway resistance syndrome. In M.H. Kryger, T. Roth \& W.C. Dement (Eds.), Principles and practice of sleep medicine (4th ed., pp. 1043-1052). Philadelphia: Elsevier Saunders.

Hakkanen, H., \& Summala, H. (2000). Sleepiness at work among commercial truck drivers. Sleep, 23(1), 49-57.

Haniffa, M., Lasserson, T. J., \& Smith, I. (2004). Interventions to improve compliance with continuous positive airway pressure for obstructive sleep apnea. The Cochrane Database of Systematic Reviews.

Harsch, I. A., Konturek, P. C., Kuehnlein, P. P., Fuchs, F. S., Pour Schahin, S., Wiest, G. H., et al. (2003). Leptin and ghrelin levels in patients with obstructive sleep apnoea: Effect of CPAP treatment. European Respiratory Journal, 22(2), 251257.

Harsch, I. A., Schahin, S. P., \& Radespiel-Troger, M., et al. (2004). Continuous positive airway pressure rapidly improves insulin sensitivity in patients with obstructive sleep apnea. American Journal of Respiratory and Critical Care Medicine, $169(2), 156-162$.

Hasler, G., Buysse, D., \& Klaghofer, R., et al. (2004). The association between short sleep duration and obesity in young adults: A 13-year prospective study. Sleep, 27, 661-666.

Hayes, A.L., Xu, F., Babineau, D., \& Patel, S.R. (2011). Sleep duration and circulating adipokine levels. Sleep, 34(2), 147-152. 
Healthy People 2010. Midcourse review: Focus area 24, respiratory diseases. Retrieved September 15, 2007, from

http://www.healthypeople.gov/data/midcourse/html/focusareas/FA24Objectives.h tm

Hedner, J., Pillar, G., Pittman, S. D., Zou, D., Grote, L., \& White, D. P. (2004). A novel adaptive wrist actigraphy algorithm for sleep-wake assessment in sleep apnea patients. Sleep, 27, 1560-1566.

Heil, D.P. (2006). Predicting activity energy expenditure using the Actical® activity monitor. Research Quarterly for Exercise and Sport, 77(1), 64-80.

Heitmann, J., Ehlenz, K., \& Penzel, T., et al. (2004). Sympathetic activity is reduced by nCPAP in hypertensive obstructive sleep apnea patients. European Respiratory Journal, 23(2), 255-262.

Henning, B., Lofgren, R., \& Sjostrom, L. (1996). A chamber for indirect calorimetry with improved transient response. Medical and Biological Engineering and Computation, 3, 207-212.

Hodgson, L. A. (1991). Why do we need sleep? Relating theory to nursing practice. Journal of Advanced Nursing, 16, 1503-1510.

Hoffstein, V., \& Szalai, J. P. (1993). Predictive value of clinical features in diagnosing obstructive sleep apnea. Sleep, 16, 118-122.

Horstman, S., Hess, C. W., Bassetti, C., Gugger, M., \& Mathis, J. (2000). Sleepinessrelated accidents in sleep apnea patients. Sleep, 23(3), 383-389.

Hoy, C. J., Vennelle, M., \& Kingshott, R. N., et al. (1999). Can intensive support improve continuous positive airway pressure use in patients with obstructive sleep apnea/hypopnea syndrome? American Journal of Respiratory and Critical Care Medicine, 1999(159), 1096-1100.

Hu, F. B., Willett, W. C., \& Colditz, G. A., et al. (1999). Prospective study of snoring and risk of hypertension in women. American Journal of Epidemiology, 150(8), 806816.

Iber, C., Ancoli-Israel, S., Chesson, A., \& Quan, S. F. (2007). AASM manual for scoring of sleep and associated events: rules, terminology and technical specification (1 st ed.). Westchester, IL: American Academy of Sleep Medicine.

Institute of Medicine Committee on Sleep Medicine and Research Board on Health Sciences Policy (2006). Sleep disorders and sleep deprivation: An unmet public health problem. Colten, H.R. \& Altevogt, B.M. (Eds.). Washington, DC: The National Academies Press. 
Ip, M. S., Lam, B., \& Laude, I. J., et al. (2001). A community study of sleep-disordered breathing in middle-aged Chinese men in Hong Kong. Chest, 119(1), 62-69.

Javaheri, S. (2006). Sleep disorders in systolic heart failure: a prospective study of 100 male patients: The final report. International Journal of Cardiology, 106, 21-28.

Jean-Louis, F., Zizi, F., Clark, L. T., Brown, C. D., \& McFarlane, S. I. (2008). Obstructive sleep apnea and cardiovascular disease: Role of the metabolic syndrome and its components. Journal of Clinical Sleep Medicine, 4(3), 261-272.

Jenkinson, C., Stradling, J., \& Peterson, S. (1997). Comparison of three measures of quality of life outcome in the evaluation of continuous positive airway pressure therapy for sleep apnoea. Journal of Sleep Research, 6, 199-204.

Kajaste, S., Brander, P. E., Telakivi, T., Patinen, M., \& Mustajoki, P. (2004). A cognitive-behavioral weight reduction program in the treatment of obstructive sleep apnea syndrome with or without initial nasal CPAP: A randomized study. Sleep Medicine, 5(2), 125-131.

Kaneko, Y., Floras, J. S., \& Usui, K., et al. (2003). Cardiovascular effects of continuous positive airway pressure in patients with heart failure and obstructive sleep apnea. New England Journal of Medicine, 348, 1233-1241.

Kapur, V., Blough, D. K., \& Snadblom, R. E., et al. (1999). The medical costs of undiagnosed sleep apnea. Sleep, 22, 225-229.

Karacan, I., \& Karatas, M. (1995). Erectile dysfunction in sleep apnea and response to CPAP. Journal of Sex and Marital Therapy, 21, 239-247.

Knapp, T. R. (1998). Quantitative nursing research. Thousand Oaks, CA: Sage Publications.

Kribbs, N. B., Pack, A. I., \& Kline, L. R., et al. (1993). Objective measurement of patterns of nasal CPAP use by patients with obstructive sleep apnea. American Review of Respiratory Disease, 147(4), 887-895.

Kripke, D. F., Ancoli-Israel, S., \& Klauber, M. R., et al. (1997). Prevalence of sleepdisordered breathing in ages 40-64 years: a population based survey. Sleep, 20 , 65-76.

Kushida, C. A. (2007). Perspectives. In C.A. Kushida (Ed.), Obstructive sleep apnea: Pathophysiology, comorbidities, and consequences (pp. 1-9). New York: Informa Healthcare USA, Inc. 
Kushida, C.A. , Chang, A., Gadkary, C., Guilleminault, C., Carrillo, O., \& Dement, W.C. (2001). Comparison of actigraphic, polysomnographic, and subjective assessment of sleep paramenteres in sleep-disordered patients. Sleep Medicine, 2, 389-396.

Lam, B., Sam, K., Mok, W. Y., Cheung, M. T., Fong, D. T., Lam, J. C., et al. (2007). Randomised study of three non-surgical treatments in mild to moderate obstructive sleep apnoea. Thorax, 62, 354-359.

Lauderdale, D. S., Knutson, K. L., \& Yan, L. L., et al. (2006). Objectively measured sleep characteristics among early-middle-aged adults: The CARDIA study. American Journal of Epidemiology, 164, 5-16.

Lavie, P., Lavie, L., \& Herer, P. (2005). All- cause mortality in males with sleep apnoea syndrome: Declining mortality rates with age. European Respiratory Journal, 25(3), 514-520.

Lee, K. A., \& Ward, T. M. (2005). Critical components of a sleep assessment for clinical practice settings. Issues in Mental Health Nursing, 26(7), 739-750.

Leinum, C.J., Dopp, J.M., \& Morgan, B.J. (2009). Sleep disordered breathing and obesity: Pathophysiology, complications and treatment. Nutrition in Clinical Practice, 24(6), 675-687.

Lichenstein, K. L., Stone, K. C., \& Donaldson, J., et al. (2006). Actigraphy validation with insomnia. Sleep, 29, 232-239.

Loube, D. I., Loube, A. A., \& Erman, M. K. (1997). Continuous positive airway pressure treatment results in weight loss in obese and overweight patients with obstructive sleep apnea. Journal of the American Dietetic Association, 97(8), 896-897.

Malhotra, A., Huang, Y., \& Fogel, R. B., et al. (2002). The male predisposition to pharyngeal collapse: Importance of airway length. American Journal of Respiratory and Critical Care Medicine, 166(10), 1388-1395.

Marti, S., Sampol, G., \& Munoz, X., et al. (2002). Mortality in severe sleep apnoea/hypopnoea syndrome patients: Impact of treatment. European Respiratory Journal, 20(6), 1511-1518.

Martinez-Garcia, M.A., Soler-Cataluna, J.J., Ejarque-Martinez, L., Soriano, Y., RomanSanchez, P., Barbe, F., Canal, J.M.M., \& Duran-Cantolla, J. Continuous positive airway pressure treatment reduces mortality in patients with ischemic stroke and obstructive sleep apnea: A 5-year follow-up study. (2009). American Journal of Critical Care Medicine, 180, 36-41. 
Matsumoto, M., Miyagishi, T., Sack, R. L., Hughes, R. J., Blood, M. L., \& Lewy, A. J. (1998). Evaluation of the Actillume wrist actigraphy monitor in the detection of sleeping and waking. Psychiatry Clinics in Neuroscience, 52, 160-161.

McArdle, N., Grove, A., \& Devereuz, G., et al. (2000). Split-night versus full-night studies for sleep apnoea/hypopnoea syndrome. European Respiratory Journal, 15, 670-675.

McCullough, P.A. (2008). Sleep/wake activity and energy expenditure in overweight and obese obstructive sleep apnea patients: a pre-and post-CPAP comparison pilot study. Unpublished manuscript.

Means, M. K., Lichstein, K. L., \& Edinger, J. D., et al. (2003). Changes in depressive symptoms after continuous positive airway pressure treatment for obstructive sleep apnea. Sleep and Breathing, 7, 31-42 .

Mehra, R., Benjamin, E. J., \& Shahar, E., et al. (2006). Association of nocturnal arrhythmias with sleep-disordered breathing: The Sleep Heart Health Study. American Journal of Respiratory and Critical Care Medicine, 173(8), 910-916.

Miller, M. A., \& Cappuccio, F. P. (2007). Inflammation, sleep, obesity and cardiovascular disease. Current Vascular Pharmacology, 5, 93-102.

Miles, P. G., Weyant, R. J., Forrest, T. D., \& Rockette, H. E. (1996). Craniofacial structure and obstructive sleep apnea syndrome: A qualitative analysis and metaanalysis of the literature. American Journal of Orthodontics and Dentofacial Orthopedics, 109(2), 163-172.

Mini Mitter. (2003). Mini Mitter physiologic research e-brief. Retrieved August 21, 2007, from Mini Mitter Web Site: http://www.minimitter.com

Minoguchi, K., Yokoe, T., \& Tazaki, T., et al. (2005). Increased carotid intima-media thickness and serum inflammatory markers in obstructive sleep apnea. American Journal of Respiratory and Critical Care Medicine, 172, 625-630.

Mokdad, A. H., Marks, J. S., \& Stroup, D. F., et al. (2004). Actual causes of death in the United States, 2000. JAMA, 291(1238-1245).

Morgenthaler, T., Alessi, C., Friedman, L., Owens, J., Kapur, V., Boehlecke, B., et al. (2007). Practice parameters for the use of actigraphy in the assessment of sleep and sleep disorders: an update for 2007. Sleep, 30(4), 519-529.

Mullis, R. M., Blair, S. N., Aronne, L. J., Bier, D. M., Denke, M. A., Dietz, W., et al. (2004). Prevention Conference VII: Obesity, a worldwide epidemic related to heart disease and stroke: Group IV: prevention/treatment. Circulation, 110(18), 484-488. 
Munro, B. H. (2005). Repeated measures analysis of variance. In B.H. Munro (Ed.), Statistical methods for health care research (5th ed., pp. 213-238). Philadelphia: Lippincott, Williams \& Wilkins.

National Center for Health Statistics. (2007). Fast stats: Overweight. Retrieved September 15, 2007, from Centers for Disease Control and Prevention Web Site: http://www.cdc.gov/nchs/fast stats/overwt.htm

National Institutes of Health (1998, September). Clinical guidelines on the identification, evaluation and treatment of overweight and obesity; The evidence report. Bethesda, MD: National Heart, Lung, and Blood Institute.

National Institutes of Health, National Heart. Lung And Blood Institute (2000, October). The practical guide: Identification, evaluation, and treatment of overweight and obesity (NIH Publication No. 00-4084). Retrieved September 15, 2007 from http://www.nhlbi.nih.gov/guidelines/obesity/ob_home.htm

National Sleep Foundation. (2002). Sleep in America poll. Retrieved March 30, 2007, from http://www.sleepfoundation.org

Nguyen, A. T., Yim, S., \& Malhotra, A. (2007). Pathogenesis. In C.A. Kushida (Ed.), Obstructive sleep apnea: Pathophysiology, comorbidities, and consequences (pp. 171-195). New York: Informa Healthcare USA, Inc.

Nieto, F. J., Young, T. B., \& Lind, B. K., et al. (2000). Association of sleep-disordered breathing, sleep apnea, and hypertension in a large community-based study: Sleep Heart Health Study. JAMA, 283(14), 1829-1836.

Niijima , M., Kimura, H., \& Edo, H., et al. (1999). Manifestation of pulmonary hypertension during REM sleep in obstructive sleep apnea syndrome. American Journal of Respiratory and Critical Care Medicine, 159(6), 1766-1772.

Noseda, A., Kempenaers, C., Kerkhofs, M., Houben, J. J., \& Linkowski, P. (1996). Sleep apnea after 1 year of domiciliary nasal-continuous positive airway pressure and attempted weight reduction: potential weaning from continuous positive airway pressure. Chest, 109, 138-143.

Oakley, N.R. (1997). Validation with polysomnography of the Sleepwatch sleep/wake scoring algorithm used by the Actiwatch activity monitoring system. Technical Report to Mini Mitter, Co., Inc.

Otake, M., Miyate, S., Noda, A., Koike, Y., Hara, Y., Sugiura, M., Minoshima, M., Kojima, J., Nakata, S., \& Nakashima, T. (2010). Monitoring sleep-wake rhythm with actigraphy in patients on continuous positive airway pressure therapy. Respiration, International Journal of Thoracic Medicine. 
Paiva, T., Farinha, A., Martins, A., \& Guilleminault, C. (1997). Chronic headaches and sleep disorders. Archives of Internal Medicine, 157, 1701-1705.

Patel, S. R., Malhotra, A., White, D. P., Gottlieb, D. J., \& Hu, F. B. (2006). Association between reduced sleep and weight gain in women. American Journal of Epidemiology, 164, 947-954.

Patel, S. R., White, D. P., \& Malhotra, A., Stanchina, M. L. \& Ayas, N.T. (2003). Continuous positive airway pressure therapy for treating sleepiness in a diverse population with obstructive sleep apnea: Results of a meta-analysis. Archives in Internal Medicine, 163, 565-571.

Peker, Y., Hedner, J., Kraiczi, H., \& Loth, S. (2000). Respiratory disturbance index: An independent predictor of morality in coronary artery disease. American Journal of Respiratory and Critical Care Medicine, 162(1), 81-86.

Penev, P. D. (2007). Sleep deprivation and energy metabolism: To sleep, perchance to eat. Current Opinion in Endocrinology, Diabetes and Obesity, 14, 374-381.

Peppard, P. E., Young, T., Palta, M., \& Skatrud, J. (2000). Prospective study of the association between sleep-disordered breathing and hypertension. New England Journal of Medicine, 342(19), 1378-1384.

Pepperell, J. C., Ramdassingh-Dow, S., \& Crosthwaite, N., et al. (2002). Ambulatory blood pressure after therapeutic and subtherapeutic nasal continuous positive airway pressure for obstructive sleep apnea: A randomised parallel trial. Lancet, 359(9302), 204-210.

Pevernagie, D. A., Demeyer, M. M., \& Claeys, S. (2005). Sleep, breathing and the nose. Sleep Medicine Review, 9(6), 437-451.

Phillips, B., \& Kryger, M. H. (2005). Management of obstructive sleep apnea-hypopnea syndrome. In M.H. Kryger, T. Roth, \& W.C. Dement (Eds.), Principles and practice of sleep medicine (4th ed., pp. 1109-1121). Philadelphia: Elsevier Saunders.

Polit, D. F., \& Beck, C. T. (2008). Nursing research: Principles and methods (8th ed.). Philadelphia: Lippincott, Williams \& Wilkins.

Punjabi, N. M., Ahmed, M. M., Polotsky, V. Y., Beamer, B. A., \& O'Donnell, C. P. (2003). Sleep-disordered breathing glucose intolerance and insulin resistance. Respiratory and Physiological Neurobiology, 136(2), 167-178.

Punjabi, N. M., \& Beamer, B. A. (2005). Sleep apnea and metabolic dysfunction. In M.H. Kryger, T. Roth \& W.C. Dement (Eds.), Principles and practice of sleep medicine (4th ed., pp. 1034-1042). Philadelphia: Elsevier Saunders. 
Ramar, K., \& Guilleminault, C. (2007). Risk factors. In C.A. Kushida (Ed.), Obstructive sleep apnea: Pathophysiology, comorbidities, and consequences (pp. 197-222). New York: Informa Healthcare USA, Inc.

Ravussin, E., \& Rising, R. (1992). Daily energy expenditure in humans: Measurements in a respiratory chamber and by double labeled water. In J.M. Kinney \& H.N. Tucker (Eds.), Energy metabolism: Tissue determinants and cellular corollaries. New York: Raven.

Rechtschaffen, A., \& Kales, A. (1968). Manual of standardized terminology, techniques and scoring system for sleep stages of human subjects. Los Angeles: Brain Information Services/Brain Information Institute.

Redenius, R., Murphy, C., O'Neill, E., Al-Hamwi, M., \& Zallek, S. (2007). Does CPAP lead to change in BMI? Sleep(30), A168.

Reichmuth, K. J., Austin, D., Skatrud, J. B., \& Young, T. (2005). Association of sleep apnea and type II diabetes: A population-based study. American Journal of Respiratory and Critical Care Medicine, 172(12), 1590-1595.

Respironics Inc. Actical. Retrieved September 15, 2007, from Respironics Web Site: http://actical.respironics.com/

Respironics, Inc. (September 21, 2006). 510(k) Summary: Actical. Retrieved September 15, 2007, from Respironics Web Site: http://actical.respironics.com/

Respironics, Inc. (2007). Actical ${ }^{\circledR}$ software version 2.1 and ActiReader instruction manual. Part Number 910-052500 Rev C.

Respironics, Inc. (2006). Actiwatch $®-16 /$ Actiwatch $®-64$, Actiwatch $®-L /$ Actiwatch $®$ Score, Actiware $₫$ 5.0/Actiware $®-C T$ 5.1: Actiwatch $®$ communication and sleep analysis software instruction manual.

Respironics, Inc. Encore ${ }^{\circledR}$ Pro SmartCard®. Retrieved April 24, 2009, from Respironics Web Site: http://encorepro.respironics.eu/

Resta, O., Foschino-Barbaro, M. P., \& Legari, G., et al. (2001). Sleep-related breathing disorders, loud snoring and excessive daytime sleepiness in obese subjects. International Journal of Obesity Related Metabolic Disorders, 25, 669-675.

Rosekind, M. R. (2006). Sleep challenges of a 24/7 global society [Monograph]. Awakening insomnia management: A collaborative approach to improved care, 15-17. 
Rothney, M.P., Schaefer, E.V., Neumann, M.M., Choi, L. \& Chen, K.Y. (2008). Validity of physical activity intensity predictions by Actigraph, Actical, and RT3 accelerometers. Obesity, 16(8), 1946-1952.

Ryan, C. F., Love, L. L., \& Buckley, P. A. (1995). Energy expenditure in obstructive sleep apnea. Sleep, 18(3), 180-187.

Sadeh, A., Hauri, P., Kripke, D., \& Lavie, P. (1995). The role of actigraphy in the evaluation of sleep disorders. Sleep, 18(4), 288-302.

Sassani, A., Findley, L. J., \& Kryger, M., et al. (2004). Reducing motor vehicle collisions, costs, and fatalities by treating obstructive sleep apnea. Sleep, 27(453458).

Schafer, H., Koehler, U., Ewig, S., Hasper, E., Tasci, S., \& Luderitz, B. (1999). Obstructive sleep apnea as a risk marker in coronary artery disease. Cardiology, 92(2), 79-84.

Schafer, H., Pauleit, D., Sudhop, T., Gouni-Berthold, I., Ewig, S., \& Berthold, H. K. (2002). Body fat distribution, serum leptin, and cardiovascular risk factors in men with obstructive sleep apnea. Chest, 122(3), 829-839.

Scharf, S. M., Seiden, L., \& Demore, J., et al. (2004). Racial differences in clinical presentations of patients with sleep-disordered breathing. Sleep \& Breathing, 8(4), 173-183.

Schroder, C. M., \& O'Hara, R. (2005). Depression and obstructive sleep apnea. Annals of General Psychiatry, 4, 13.

Schwab, R. J., Gupta, K. B., Gefter, W. B., Metzger, L. J., Hoffman, E. A., \& Pack, A. I. (1995). Upper airway and soft tissue anatomy in normal subjects and patients with sleep-disordered breathing: Significance of the lateral pharyngeal walls. American Journal of Respiratory and Critical Care Medicine, 152(5), 1673-1689.

Schwartz, D. J., Kohler, W. C., \& Karatinos, G. (2005). Symptoms of depression in individuals with obstructive sleep apnea may be amenable to treatment with continuous positive airway pressure. Chest, 128(3), 1304-1309.

See, C. Q., Mensah, E., \& Olopade, C. O. (2006). Obesity, ethnicity, and sleep-disordered breathing: Medical and health policy implications. Clinics in Chest Medicine, 27(3), 521-533.

Sharar, E., Whitney, C. W., \& Redline, S., et al. (2001). Sleep-disordered breathing and cardiovascular disease: Cross-sectional results of the Sleep Heart Health Study. American Journal of Respiratory and Critical Care Medicine, 163, 19-25. 
Shepertycky, M. R., Banno, K., \& Kryger, M. H. (2005). Differences between men and women in the clinical presentation of patients diagnosed with obstructive sleep apnea syndrome. Sleep, 28(3), 309-314.

Shimzu, K., Chin, K., \& Nakamura, T., et al. (2002). Plasma leptin levels and cardiac sympathetic function in patients with obstructive sleep apnea-hypopnea syndrome. Thorax, 57, 429-434.

Shneerson, J., \& Wright, J. (2007). Lifestyle modification for obstructive sleep apnoea. Cochrane Database of Systematic Reviews, 1, 1-7.

Sin, D. D., Mayer, I., Man, G. C., Ghahary, A., \& Pawluk, L. (2002). Can continuous positive airway pressure therapy improve the general health status of patients with obstructive sleep apnea? A clinical effectiveness study. Chest, 122(5), 1679-1685.

Somers, V. K., \& Javaheri, S. (2005). Cardiovascular effects of sleep-related breathing disorders. In M.H. Kryger, T. Roth \& W.C. Dement (Eds.), Principles and Practice of Sleep Medicine (4th ed., pp. 1180-1191). Philadelphia: Elsevier Saunders.

Spiegel, K., Leproult, R., \& Van Cauter, E. (1999). Impact of sleep debt on metabolic and endocrine function. Lancet, 354(9188), 1435-1439.

Spiegel, K., Tasali, E., Penev, P., \& Van Cauter, E. (2004). Brief communication: Sleep curtailment in health young men is associated with decreased leptin levels, elevated ghrelin levels and increased hunger and appetite. Annals of Internal Medicine, 141, 846-850.

SPSS, Inc. (2008). SPSS Statistics 17.0 for Windows. SPSS, Inc. Chicago, IL.

Stenlof, K., Grunstein, R., Hedner, J., \& Sjostrom, L. (1996). Energy expenditure in obstructive sleep apnea: Effects of treatment with continuous positive airway pressure. The American Journal of Physiology, 271, E1036-E1043.

Stevens, J. P. (2002). Applied multivariate statistics for the social sciences (4th ed.). Mahwah, NJ: Lawrence Erlbaum Associates.

Stoohs, R. A., Facchini, F. S., \& Philip, P., et al. (1993). Selected cardiovascular risk factors in patients with obstructive sleep apnea: Effect of continuous positive airway pressure therapy. Sleep, 16, S141-S142.

Strohl, K. P., \& Redline, S. (1986). Nasal CPAP therapy, upper airway muscle activation and obstructive sleep apnea. American Review of Respiratory Disease, 134, 555558 . 
Stunkard, A. J., \& Wadden, T. A. (1993). Obesity Theory and therapy (2nd ed.). New York: Raven Press.

Sullivan, C. E., Issa, F. G., \& Berthon-Jones, M., et al. (1981). Reversal of obstructive sleep apnea by continuous positive airway pressure applied through the nares. Lancet, 1, 862-865.

Summerfield, L. M. (2001). Nutrition, exercise, and behavior: an integrated approach to weight management. Belmont, CA: Wadsworth/Thompson Learning.

Taheri, L., Lin, L., Austin, D., Young, T., \& Mignot, E. (2004). Short sleep duration is associated with reduced leptin, elevated ghrelin, and increased body mass index. PLoS Medicine, 1, 62.

Tasali, E., \& Van Cauter, E. (2002). Sleep-disordered breathing and the current epidemic of obesity: Consequences or contributing factor. American Journal of Respiratory and Critical Care Medicine, 165(5), 562-563.

Traviss, K. A., Barr, S. I., Fleming, J. A., \& Ryan, C. F. (2002). Lifestyle-related weight gain in obese men with newly diagnosed obstructive sleep apnea. Journal of the American Dietetic Association, 102(5), 703-706.

Tung, A. (2005). The biology and genetics of obesity and obstructive sleep apnea. Anesthesiology Clinics in North America, 23(3), 445-461.

U.S. Department of Health and Human Services (2001). The Surgeon General's call to action to prevent and decrease overweight and obesity. Rockville, MD: U.S. Department of Health and Human Services, Public Health Service, Office of the Surgeon General.

U.S. Department of Health and Human Services, Centers for Disease Control and Prevention. (July 27, 2007). Behavioral Risk Factor Surveillance: U.S. obesity trends, 1985-2006. Retrieved September 15, 2007 from:

http://www.cdc.gov/nccdphp/dnpa/obesity/trend/maps/

U.S. Department of Health and Human Services, Centers for Disease Control and Prevention. (March 3, 2011). Behavioral Risk Factor Surveillance: U.S. obesity trends, 1985-2009. Retrieved June 16, 2011 from:

http://www.cdc.gov/obesity/data/trends.html

U.S. Department of Health and Human Services, National Institutes of Health, National Heart, Lung, and Blood Institute, \& National Center on Sleep Disorders Research. (2003, July). 2003 National sleep disorders research plan (NIH Publication No. 03-5209). 
Vallieres, A., \& Morin, C. M. (2003). Actigraphy in the assessment of insomnia. Sleep, 26, 902-906.

Vaughn, B. V., \& D'Cruz, O. F. (2005). Cardinal manifestations of sleep disorders. In Kryger, M.H., Roth, T. \& Dement, W.C. (Eds.), Principles and practice of sleep medicine (4th ed., pp. 594-601). Philadelphia: Elsevier Saunders.

Veasey, S. C., Guilleminault, C., Strohl, K. P., Sanders, M. H., Ballard, R. D., \& Magalang, U. J. (2006). Medical therapy for obstructive sleep apnea: A review by the medical therapy for obstructive sleep apnea task force of the Standards of Practice Committee of the American Academy of Sleep Medicine. Sleep, 29(8), 1036-1044.

Vgontas, A. N., Bixler, E. O., \& Chrousos, G. P. (2005). Sleep apnea is a manifestation of the metabolic syndrome. Sleep Medicine Review, 9(3), 211-224.

Vgontas, A. N., Papanicolaou, D. A., \& Bixler, E. O., et al. (2000). Sleep apnea and daytime sleepiness and fatigue: Relation to visceral obesity, insulin resistance, and hypercytokinemia. Journal of Clinical Endocrinology and Metabolism, 85(3), 1151-1158.

Vgontzas, A. N., Tan, T. L., Bixler, E. O., Martin, L. F., Shubert, D., \& Kales, A. (1994). Sleep apnea and sleep disruption in obese patients. Archives of Internal Medicine, 154(15), 1705-1711.

Walker-Engstrom, M. A., Tegelberg, A., Wilhelmsson, B., \& Ringqvist, I. (2002). 4-year follow-up of treatment with dental appliance or uvulopalatopharyngoplasty in patients with obstructive sleep apnea: a randomized study. Chest, 121, 739-746.

Walsh, J. K., Dement, W. C., \& Dinges, D. F. (2005). Sleep medicine, public policy, and public health. In M.H. Kryger, T. Roth \& W.C. Dement (Eds.), Principles and practice of sleep medicine (4th ed., pp. 648-658). Philadelphia: Elsevier Saunders.

Weaver, T. E. (2001). Outcome measurement in sleep medicine practice and research. Part I: Assessment of symptoms, subjective and objective daytime sleepiness, health-related quality of life and functional status. Sleep Medicine Review, 5, 103128.

Weaver, T. E., \& George, C. F. (2005). Cognition and performance in patients with obstructive sleep apnea. In M.H. Kryger, T. Roth \& W.C. Dement (Eds.), Principles and practice of sleep medicine (4th ed., pp. 1023-1033). Philadelphia: Elsevier Saunders.

Weaver, T. E., Kribbs, N. B., \& Pack, A. I., et al. (1997). Night-to-night variability in CPAP use over the first three months of treatment. Sleep, 20, 278-283. 
West, S. D., Kohler, M., Nicoll, D. J., \& Strading, J. R. (2008). The effect of continuous positive airway pressure treatment on physical activity in patients with obstructive sleep apnoea: A randomized controlled trial. Sleep Medicine, 10(9), 1056-1058.

Wilson, P. W., D'Agostino, R. B., Sullivan, L., Parise, H., \& Kannel, W. B. (2002). Overweight and obesity as determinants of cardiovascular risk: the Framingham experience. Archives of Internal Medicine, 162(16), 1867-1872.

Winslow, D. H., Bowden, C. H., DiDonato, K., McCullough, P. A., Day, W. W. (2010). A randomized, double-blind, placebo-controlled, parallel-group study of Phentermine/Topiramate CR (VI-0521) for the treatment of obstructive sleep apnea/hypopnea syndrom in obese adults [Abstract]. Sleep Abstract Supplement, Abstract No. 0475.

Wittig, R. M., Romaker, A., \& Zorick, F. J., et al. (1984). Night-to-night consistency of apneas during sleep. American Review of Respiratory Disease, 129, 244-248.

Wolk, R., Shamsuzzaman, A. S., \& Somers, V. K. (2003). Obesity, sleep apnea, and hypertension. Hypertension, 42(6), 1067-1074.

Wolf, A. M. (1998). What is the economic case for treating obesity? Obesity Research, 6, 2S-7S.

Wolf, A. M., \& Colditz, G. A. (1998). Current estimate of the economic cost of obesity in the United States. Obesity Research, 6(2), 97-106.

Wolk, R., \& Somers, V. K. (2006). Obesity-related cardiovascular disease: Implications of obstructive sleep apnea. Diabetes Obesity and Metabolism, 8(3), 250-260.

Won, C., \& Robert, D. (2007). Morbidity and mortality. In C.A. Kushida (Ed.), Obstructive sleep apnea: pathophysiology, comorbidities and consequences (pp. 259-274). New York: Informa Healthcare USA, Inc.

Yamamoto, H., Akashiba, T., \& Kosaka, N., et al. (2000). Long-term effects of nasal continuous positive airway pressure on daytime sleepiness, mood, and traffic accidents in patients with obstructive sleep apnoea. Respiratory Medicine, 94(1), 87-90.

Young, T., Blustein, J., Finn, L., \& Palta, M. (1997). Sleep-disordered breathing and motor vehicle accidents in a population-based sample of employed adult. Sleep, 20(8), 608-613.

Young, T., Palta, M., \& Dempsey, J., Skatrud, J., Weber, S. \& Badr, S. (1993). The occurrence of sleep-disordered-breathing among middle aged adults. New England Journal of Medicine, 328, 1230-1235. 
Young, T., Peppard, P. E., \& Gottlieb, D. J. (2002). Epidemiology of obstructive sleep apnea: a population perspective. American Journal of Respiratory and Critical Care Medicine, 165, 1217-1239.

Zozula, R., \& Rosen, R. (2001). Compliance with continuous positive airway pressure therapy: Assessing and improving treatment outcomes. Current Opinion in Pulmonary Medicine, 7, 391-398. 


\section{Appendix A}

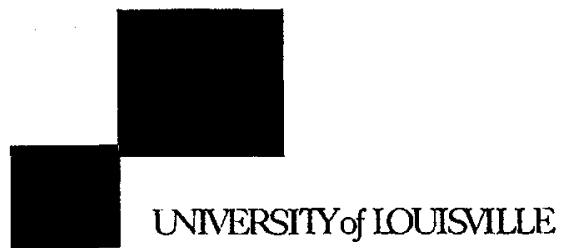

INSTITUTIONAL REVIEW BOARDS

University of Louisville

MedCenter One, Suite 200

501 E. Broadway

Louisvilie, Kentucky $40202-1798$

Office: $\quad 502-852-5188$

Fax: $\quad 502-852-2164$

To: $\quad$ Speck, Barbara

From: The University of Louisville Institutional Review Board (IRB)

Date: Tuesday, July 28, 2009

Subject: $\quad$ Approval Letter

Tracking \#: AMEND-1396 (08.0173)

Title: $\quad$ Sleep/Wake Activity and Energy Expenditure in Overweight and

Obese Adults with Obstructive Sleep Apnea: A Pre- and Post-CPAP

Comparison

The amendment request has been received by the Human Subjects Protection Program Office and approved by the chair/vice chair of the Institutional Review Board (IRB) on $7 / 27 / 2009$ through the expedited review procedure according to 45 CFR 46.110

(B). The following items were reviewed and approved:

- Revised HIPAA Research Authorization \& Revocation

- Revised HIPAA Partial Waiver

The amendment modifications include:

- The protocol was changed from a pilot study of 3 to 5 subjects, to a dissertation study requiring 34 enrolled subjects.

- Additional changes include an extra timepoint for data collection and requires the study subjects to participate for a longer period.

The committee will be advised of this action at their next full board meeting. The stamped approved study document(s) will follow in a general notification. Please begin using your newly approved (stamped) consent(s) at this time. The previous versions are no longer valid. If you need assistance in accessing any of the study documents, please feel free to contact our office at (502) 852-5188. You may also email our service account at hsppofc@louisville.edu for assistance.

Thank you. 


\section{quk 2. Doll}

Board Designee: Goldman, Julie

Continue following the regulations below:

1. Unanticipated problems or serious adverse events encountered in this research study must be reported to the IRB within five (5) work days.

2. Any modifications to the study protocol or informed consent form must be reviewed and approved by the IRB prior to implementation.

3. You may not use a modified informed consent form until it has been approved and validated by the IRB.

Letter Sent By: Perkins, Erin, 7/28/2009 8:00 AM 


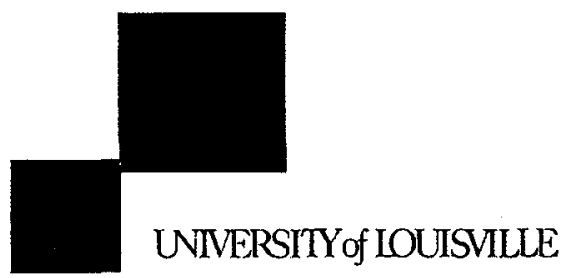

\begin{tabular}{l} 
INSTITUTIONAL REVIEW BOARDS \\
\hline University of Louisville \\
MedCenter One, Suite 200 \\
501 E. Broadway \\
Louisville, Kentucky $40202-1798$ \\
Office: $\quad 502-852-5188$ \\
Fax: $\quad 502-852-2164$
\end{tabular}

\title{
Expedited - Continuing Review - Approval
}

\author{
To: $\quad$ Speck, Barbara \\ From: $\quad H u m a n$ Subjects Protection Program Office \\ Date: $\quad$ Tuesday, April 13, 2010 \\ Subject: No action required
}

Tracking \#: CR-620 (08.0173)

Title: $\quad$ Sleep/Wake Activity and Energy Expenditure in Overweight and Obese Adults with Obstructive Sleep Apnea: A Pre- and Post-CPAP Comparison

The continuation request for the above study was reviewed by the Chair of the Institutional Review Board (IRB) through the expedited review procedure, according to 45 CFR 46.110 and 21 CFR 56.110, since this study falls under Expedited Category (4) Collection of data through noninvasive procedures (not involving general anesthesia or sedation) routinely employed in clinical practice, excluding procedures involving $x$-rays or microwaves. Where medical devices are employed, they must be cleared/approved for marketing.

The following documents were approved along with the continuation request:

- Research Protocol, not dated

- Informed Consent, not dated

- Protocol Synopsis, not dated

This study now has continued committee approval from 05/09/2010 through 05/08/2011. This action will be reported promptly to the IRB at a scheduled full Board meeting.

The following is a link to an Instruction Sheet for BRAAN2 "How to Locate Stamped/Approved Documents in BRAAN2":

https://louisville.edu/research/braan2/help/Docs.pdf

Please begin using your newly approved (stamped) consent(s) at this time. The previous versions are no longer valid. If you need assistance in accessing any of the study documents, please feel free to contact our office at (502) $852-5188$. You may also email our service account at hsppofc@louisville.edu for assistance. 
Thank you.<smiles>CCCCCCC=CC1C=CC(C)CC1</smiles>

Board Designee: Martinez, Serge

Letter Sent By: Block, Sherry, 4/13/2010 8:34 AM

Continue following the regulations below:

1. Unanticipated problems or serious adverse events encountered in this research study must be reported to the IRB within five (5) working days.

2. Any modifications to the study protocol or informed consent form must be reviewed and approved by the IRB prior to implementation.

3. You may not use a modified informed consent form until it has been approved and validated by the IRB.

Full Accreditation since June 2005 by the Association for the Accreditation of Human Research Protection Programs, Inc. 


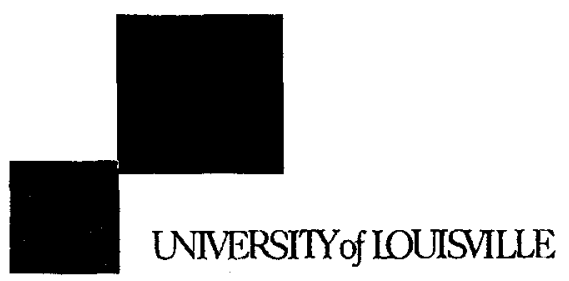

INSTITUTIONAL REVIEW BOARDS

University of Louisville

MedCenter One, Suite 200

501 E. Broadway

Louisvilie, Kentucky 40202-1798

Office: $\quad 502-852-5188$

Fax: $\quad 502-852-2164$

\section{Expedited - Continuing Review - Approval}

To:

Speck, Barbara

From: Human Subjects Protection Program Office

Date: $\quad$ Tuesday, April 19, 2011

Subject: No action required

Tracking \#: CR-1216 (08.0173)

Title: $\quad$ SleepMake Activity and Energy Expenditure in Overweight and Obese Adults with Obstructive Sleep Apnea: A Pre- and Post-CPAP Comparison

The continuation request for the above study was reviewed by the Chair of the Institutional Review Board (IRB) through the expedited review procedure, according to 45 CFR 46.110 and 21 CFR 56.110, since this study falls under Expedited Category (4) Collection of data through noninvasive procedures (not involving general anesthesia or sedation) routinely employed in clinical practice, excluding procedures involving $x$-rays or microwaves. Where medical devices are employed, they must be cleared/approved for marketing.

The following documents were approved along with the continuation request:

- Research Protocol, not dated

This study now has continued committee approval from 05/09/2011 through 05/08/2012. This action will be reported promptly to the IRB at a scheduled full Board meeting.

Thank you.

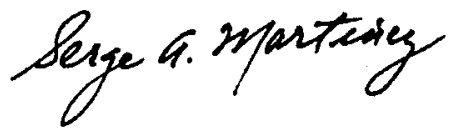

Board Designee: Martinez, Serge

Letter Sent By: Block, Sherry, 4/19/2011 11:03 AM 
Continue following the regulations below:

1. Unanticipated problems or serious adverse events encountered in this research study must be reported to the IRB within five (5) working days.

2. Any modifications to the study protocol or informed consent form must be reviewed and approved by the IRB prior to implementation.

3. You may not use a modified informed consent form until it has been approved and validated by the IRB.

Full Accreditation since June 2005 by the Association for the Accreditation of Human Research Protection Programs, Inc.

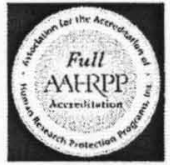


Appendix B

Actical ${ }^{\circledR}$ Report

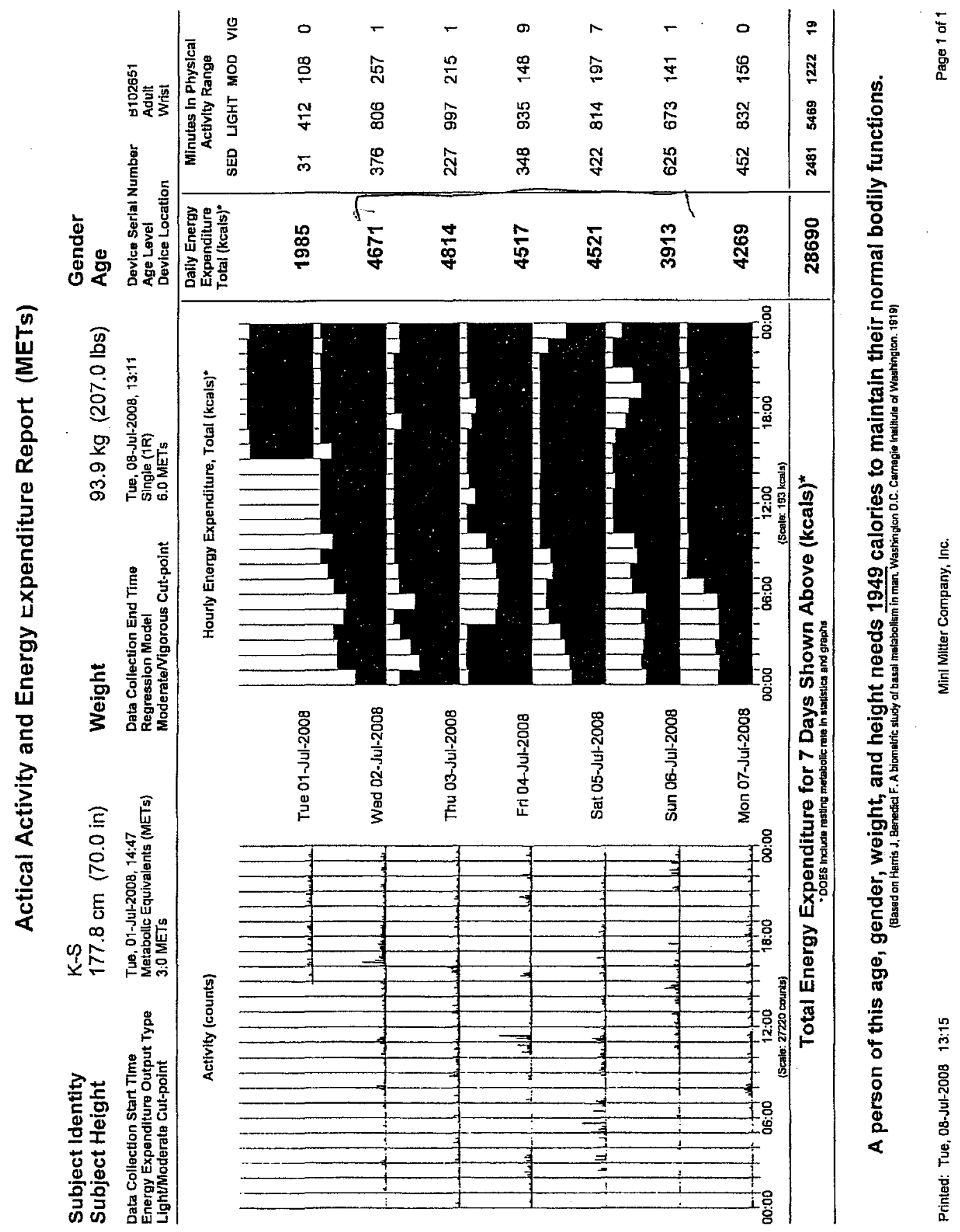


Appendix C

Actiwatch ${ }^{\circledR}$ Report

Actiware Print Report

Analysis Name: New Analysis

Subject ID: $\quad \mathrm{K}-\mathrm{S} 002$

Date of Birth:

Data Collection End: $7 / 8 / 2008,1: 16: 30 \mathrm{PM} \quad$ Actiwatch SN: V963450

Data Collection Start: 7/1/2008, 2:53:00 PM

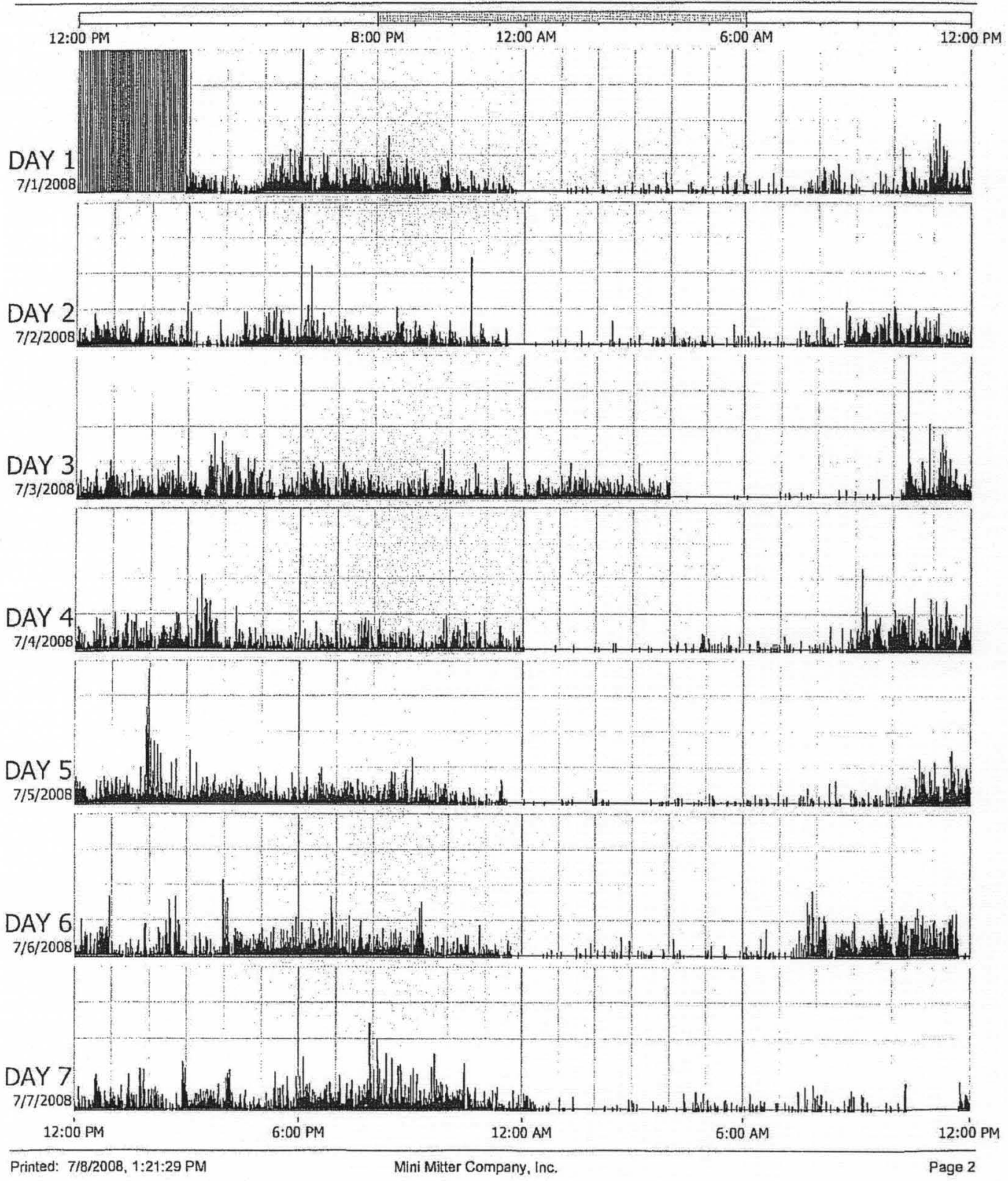




\section{Actiware Print Report}

Analysis Name: New Analysis Subject ID: K-S 002

Data Collection Start: 7/1/2008, 2:53:00 PM
Date of Birth:

Data Collection End: 7/8/2008, 1:16:30 PM
Gender:

Actiwatch SN: V963450

\section{Daily Interval Statistics}

\begin{tabular}{|c|c|c|c|c|c|c|c|}
\hline Day\# & Start Date & Start Time & End Date & End Time & $\frac{\text { Total AC }}{\text { (counts) }}$ & $\frac{\text { Avo AC/min }}{\text { (counis/minute) }}$ & $\frac{\text { Max AC }}{\text { (counts) }}$ \\
\hline 1 & $7 / 1 / 2008$ & 2:53:00 PM & $7 / 2 / 2008$ & $12: 00: 00 \mathrm{PM}$ & $264330^{\circ}$ & 208.63 & 1448 \\
\hline 2 & $7 / 2 / 2008$ & 12:00:00 PM & $7 / 3 / 2008$ & 12:00:00 PM & 297981 & 206.93 & 1858 \\
\hline 3 & $7 / 3 / 2008$ & 12:00:00 PM & $7 / 4 / 2008$ & $12: 00 ; 00 \mathrm{PM}$ & 406074 & 282.00 & 3054 \\
\hline 4 & $7 / 4 / 2008$ & 12:00:00 PM & $7 / 5 / 2008$ & $12: 00: 00 \mathrm{PM}$ & 378322 & 262.72 & 1746 \\
\hline 5 & $7 / 5 / 2008$ & 12:00:00 PM & $7 / 6 / 2008$ & $12: 00: 00 \mathrm{PM}$ & 328551 & 228.16 & 2870 \\
\hline 6 & $7 / 6 / 2008$ & 12:00:00 PM & $7 / 7 / 2008$ & 12:00:00 PM & 424050 & 294.48 & 7640 \\
\hline 7 & $7 / 7 / 2008$ & $12: 00: 00 \mathrm{PM}$ & $7 / 8 / 2008$ & $12: 00: 00 \mathrm{PM}$ & 299030 & 207.66 & 1858 \\
\hline 8 & $7 / 6 / 2008$ & 12:00:00 PM & $7 / 8 / 2008$ & 1:16:30 PM & 5572 & 72.84 & 723 \\
\hline n & & t & & $\Delta$ & & 8 & \\
\hline Average(n) & * & * & * & * & 300488.76 & 220.43 & 1899.62 \\
\hline Std Dev(n-t) & * & • & * & & 131775.19 & 69.07 & 751.86 \\
\hline Davi* & \%inualld AC & $\frac{\% \text { lnvalid SW }}{6(\%)}$ & Wake Time & \%Wake & Sleep Time & \% Sieen & \\
\hline 1 & 0.00 & 0.16 & 73400 & 58.02 & 531.00 & 41.98 & \\
\hline 2 & 0.00 & 0.00 & 934.00 & 64.86 & 505.00 & $35: 14$ & \\
\hline 3 & 0.00 & 0.00 & 1067.00 & 74.10 & 373.00 & 25.90 & \\
\hline 4 & 0.00 & 0.00 & 380.50 & 68.09 & 459.50 & 31.91 & \\
\hline 5 & 0.00 & 0.00 & 870.00 & 60.42 & 570.00 & 39.58 & \\
\hline 6 & 0.00 & 0.00 & 685.50 & 68.44 & 454.50 & 31.56 & \\
\hline 7 & 0.00 & 0.00 & 870.00 & 60.42 & 570.00 & 39.58 & \\
\hline 8 & 0.00 & 2.61 & 15.00 & 20.13 & 59.50 & 79.87 & \\
\hline$\pi$ & & 8 & $\theta$ & & & & \\
\hline Average(n) & 0.00 & 0.35 & 807.00 & 59.31 & 440.44 & 40.69 & \\
\hline Std Dev(n-1) & 0.00 & 0.92 & 336.14 & 16.69 & 167.46 & 16.69 & \\
\hline
\end{tabular}


Appendix D

Subject Information Log

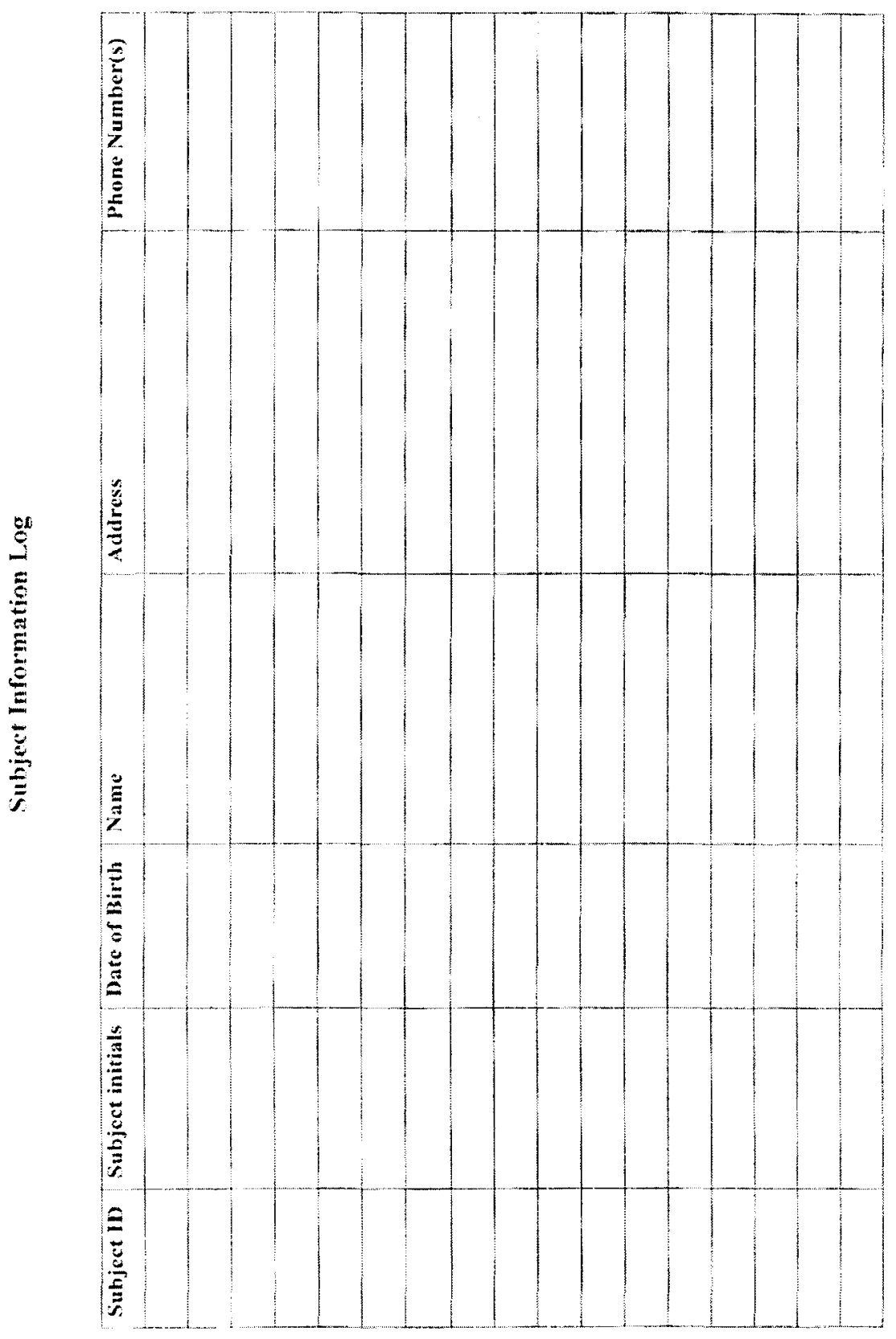




\section{Appendix E}

Obstructive Sleep Apnea Polysomnography Data

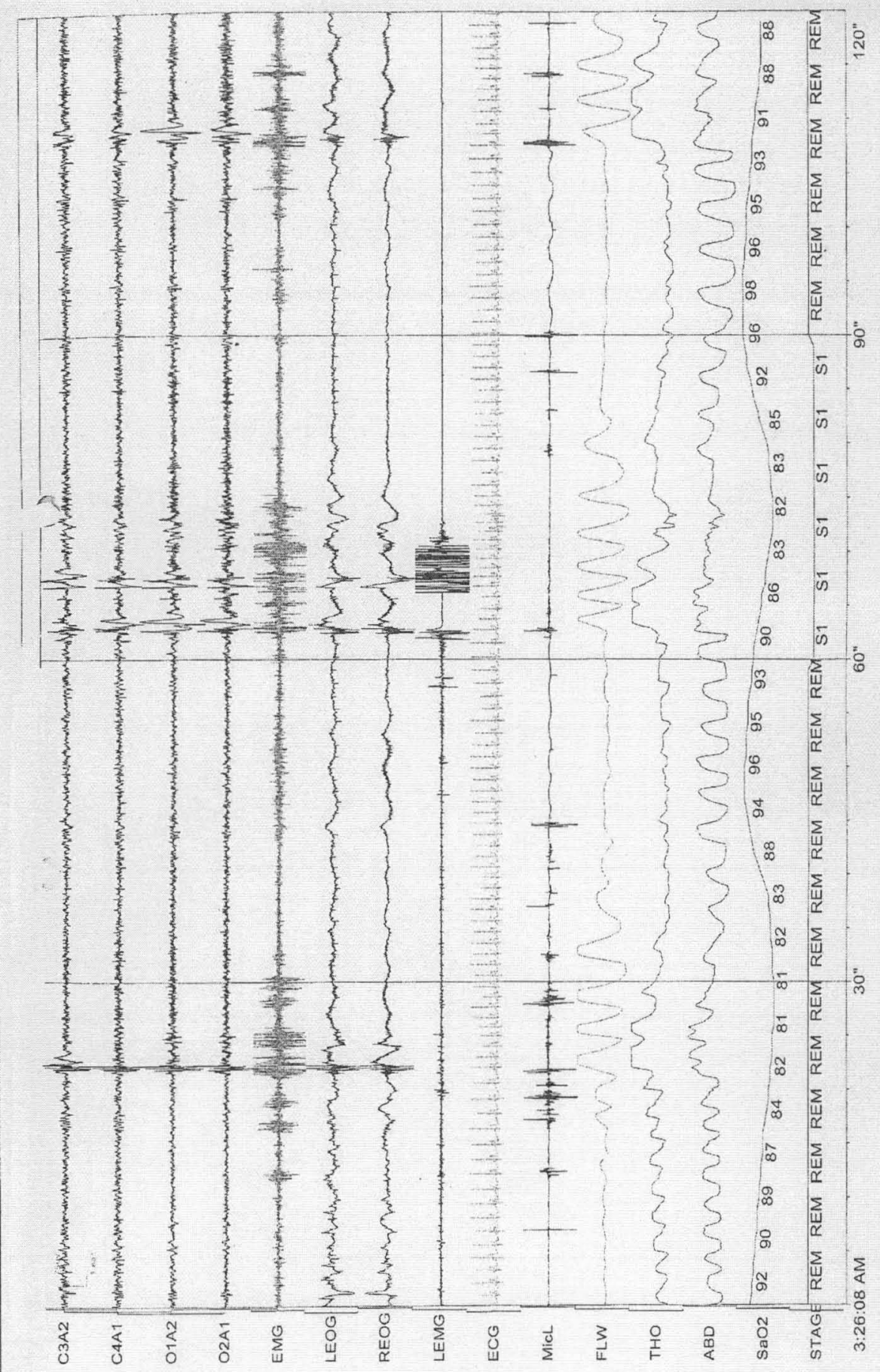




\section{Appendix F}

\section{CPAP Titration Polysomnography Data}

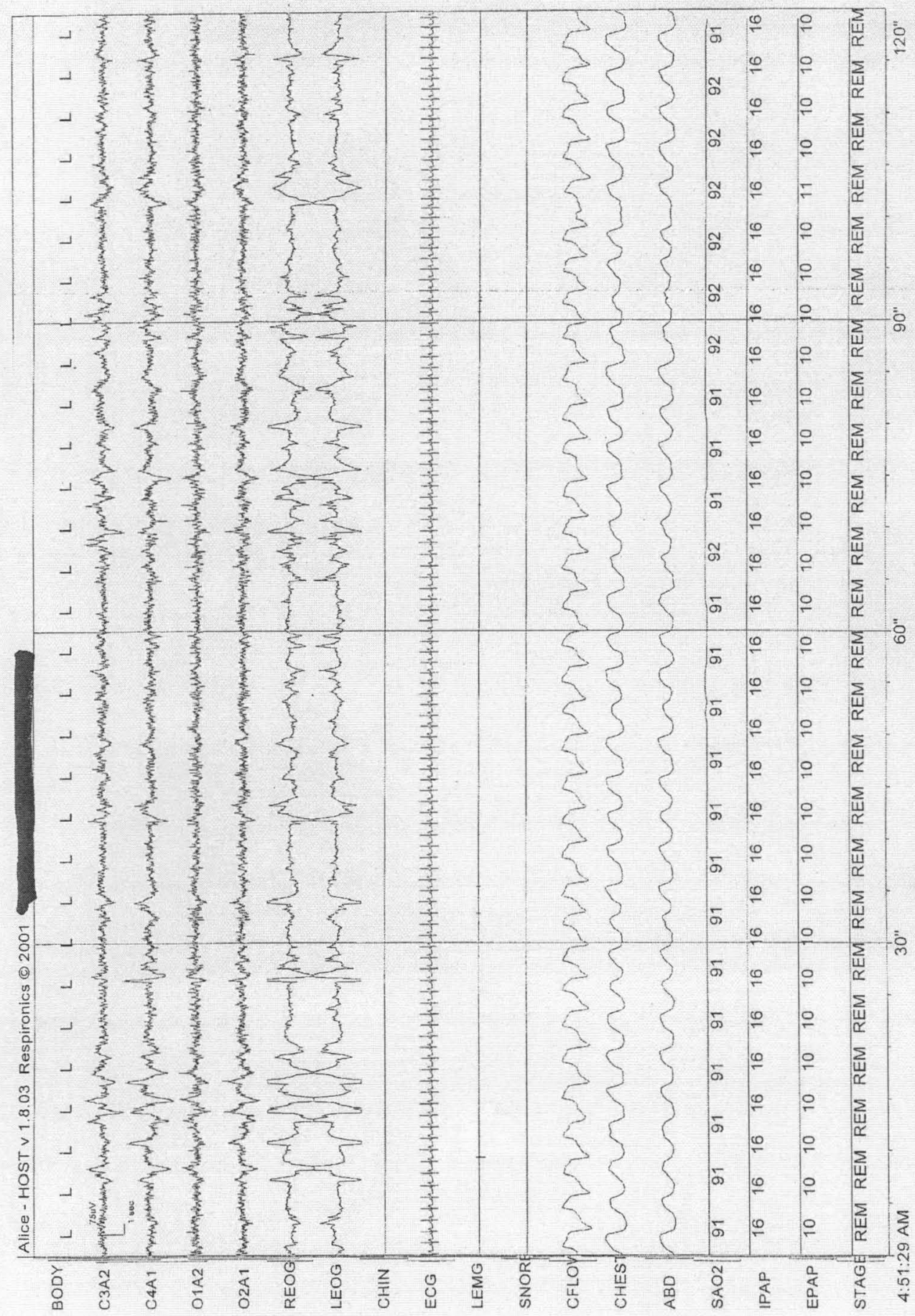




\title{
Appendix G
}

\section{Obstructive Sleep Apnea Polysomnography Report}

\author{
Sleep Medicine Specialists
}

\author{
1169 Eastern Parkway, Suite \#3357 \\ Louisville, Kentucky $\mathbf{4 0 2 1 7}$ \\ Phone 502.454.0755 \\ Fax 502.454.3497
}

Polysomnogram (PSG)

\begin{tabular}{|c|c|c|c|}
\hline $\begin{array}{l}\text { Patient nan } \\
\text { Test date: } \\
\text { Test type: }\end{array}$ & Adult & $\begin{array}{l}\text { Date of bitth: } \\
\text { Physician: } \\
\text { Axis A/ICSD: }\end{array}$ & $\begin{array}{l}\text { Dr. David Winslow } \\
780.53-0\end{array}$ \\
\hline Study \#: & 9080 & Patient ID \#: & 120922 \\
\hline
\end{tabular}

Dear

We appreciate your referral of this patient to our services for Polysomnography. The 16 channel overnight study consists of a combination of EEG, Chin EMG, EOG, leg EMG, thoracic and abdominal wall movements, nasal/oral airflow, ECG, SaO2, body position, and trocheal sound. The results are as follows:

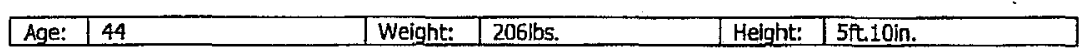

Medications: Reglan,Protonix, Lexapro

Sleep Staging

Lights Out: 11:04:47 PM Lights On: 1:16:47 AM

\begin{tabular}{|l|r|l|l|l|l|}
\hline Time in Bed (TB): & 132.0 & $\mathrm{~min}$. & Sleep Onset (from L Out): & 15.5 & $\mathrm{~min}$. \\
\hline Total Sleep Time: & 113.0 & $\mathrm{~min}$. & REM Onset (from Sleep Onset): & & $\mathrm{min}$. \\
\hline Sleep Efficiency: & $\mathbf{8 5 . 6}$ & $\%$ & & \\
\hline
\end{tabular}

\begin{tabular}{|c|c|c|c|c|c|}
\hline Sleep stage & \multicolumn{2}{|c|}{ Sleep duration } & \multicolumn{2}{|c|}{$\%$ of time in bed } & Normal values \\
\hline Stage N1 & 2.5 & min. & 1.9 & $\%$ & $(5 \%)$ \\
\hline Stage N2 & 99,0 & $\min$. & 75.0 & $\%$ & $(50 \%)$ \\
\hline Stage N3 (delta) & 11.5 & $\min$. & 8.7 & $\%$ & $(15 \%)$ \\
\hline Stage R (REM) & 0.0 & min. & 0.0 & $\%$ & $(20 \%)$ \\
\hline Stage W (wake) & 19.0 & min. & 14.4 & $\%$ & $(10 \%)$ \\
\hline
\end{tabular}

Respiratory

\begin{tabular}{|l|c|c|c|c|c|}
\cline { 2 - 6 } \multicolumn{1}{c|}{} & \multicolumn{3}{c|}{ Apneas } & \multicolumn{1}{c|}{} \\
\cline { 2 - 6 } \multicolumn{1}{c|}{} & Central & Obstructive & Mixed & Hypopneas & Total \\
\hline Mean duration & 0 & 42 & 0 & 113 & 155 \\
\hline Max. duration & 0.0 & 18.9 & 0.0 & 21.3 & \\
\hline
\end{tabular}

\begin{tabular}{|c|c|c|c|}
\hline $\begin{array}{c}\text { Apnea/Hypopnea Index } \\
\text { (AHI) }\end{array}$ & Total Index & REM Index & Non-REM Index \\
\cline { 2 - 4 } & $\mathbf{8 2 . 3}$ & & $\mathbf{8 2 . 3}$ \\
\hline
\end{tabular}




\begin{tabular}{|c|c|c|c|}
\hline \multicolumn{4}{|l|}{ Body Position } \\
\hline & Duration (min.) & $\%$ asleep & $\mathrm{AHI}$ \\
\hline \multicolumn{4}{|l|}{$\mathrm{Up}$} \\
\hline Supine & 80.3 & 97.8 & 89.4 \\
\hline \multicolumn{4}{|l|}{ Left Side } \\
\hline \multicolumn{4}{|l|}{ Supine Left } \\
\hline \multicolumn{4}{|l|}{ Prone Rlght } \\
\hline \multicolumn{4}{|l|}{ Prone } \\
\hline \multicolumn{4}{|l|}{ Supine Right } \\
\hline Right Side & 36.2 & 95.3 & 66.1 \\
\hline
\end{tabular}

Oximetry

\begin{tabular}{|l|l|l|l|l|l|}
\hline Average 02 while awake & 96 & $\%$ & Minimum 02 Value & 77 & $\%$ \\
\hline Ancrag
\end{tabular}

\begin{tabular}{|l|c|c|}
\hline Average O2 while awake & 96 & $\%$ \\
\hline Average 02 whlle in REM & & $\%$ \\
\hline Average 02 while in Non-REM & 93 & $\%$ \\
\hline
\end{tabular}

Average $\mathrm{O} 2$ while in Non-REM

Heart Rate
\begin{tabular}{|l|c|c|c|}
\hline Mean Heart Rate (BPM) & Wake & REM & Non-REM \\
\hline Lowest Heart Rate (BPM) & 74.6 & & 67.0 \\
\hline Highest Heart Rate (BPM) & 55 & & 46 \\
\hline
\end{tabular}

Periodic Limb Movements (legs unless otherwise noted)

Total number of limb movements

Mean duration of limb episodes

Arousals assodiated with PLMS

Total number of WK or MVT episodes

Arousal index

$\%$ of pages with arousal during sleep

PLM index (\#/hr)

\begin{tabular}{l|l} 
Total sleep time with & $0.0 / \mathrm{hr}$
\end{tabular} 0

$: 6$

87.6/hr (sleep)

$: 71.2$

Page 2 


\title{
Sleep.Medicine Specialists
}

\author{
1169 Eastern Parkway, Suite \#3357 \\ Louisville, Kentucky 40217 \\ Phone 502.454.0755 \\ Fax 502.454.3497
}

Polysomnogram (PSG)

Re:

Study date:

The patient tolerated NPSG well with loud snoring and OSA observed. There were a total of 155 respiratory abnormalities with an apnea/hypopnea index (AHI) of $\mathbf{8 2 , 3}$ (average number of apneas/ hypopneas per hour of sleep-a value greater than 5.0 is considered abnormal). Oxygen saturation dropped to $77 \%$ during the recording. ECG recording revealed no ECG abnormalities. The patient will follow up with Dr. David Winslow for sleep study results.

Physician's Impression: Very Severe Obstructive Sleep Apnea Syndrome

Physician's Signature:

dNm 


\section{Appendix $\mathrm{H}$}

\section{CPAP Titration Polysomnography Report}

\section{Sleep Medicine Specialists}

1169 Eastern Parkway, Suite \#3357

Louisville, Kentucky $\mathbf{4 0 2 1 7}$

Phone 502.454.0755

Fax 502.454.3497

\section{CPAP Titration}

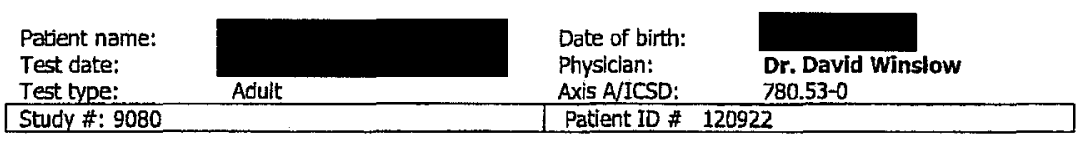

Referring Physician:

Age: 44 years

Medications: Reglan, Protonli, Lexapro

\section{Sleep Staging}

Lights Out: 1:18:47 AM

Light On: 6:01:47 AM

\begin{tabular}{|l|r|l|l|r|l|}
\hline Time in Bed (TIB): & $\mathbf{2 8 3 . 0}$ & $\mathrm{min}$. & Sleep Onset (from L. Out): & $\mathbf{1 1 . 0}$ & $\mathrm{min}$. \\
\hline Total Sleep Tme: & $\mathbf{2 6 0 . 5}$ & min. & REM Onset (from Sleep Onset): & $\mathbf{9 3 . 5}$ & $\mathrm{min}$. \\
\hline Sleep Efficiency: & $\mathbf{9 2 . 0}$ & $\%$ & & \\
\hline
\end{tabular}

\begin{tabular}{|c|c|c|c|c|c|c|c|c|}
\hline Sleep stage & Sleep dur & tion & $\%$ of time & in $b$ & & Nomal values & & \\
\hline Stage N1 & 9.5 & min. & & 3.4 & $\%$ & $(5 \%)$ & & \\
\hline Stage N2 & 146.0 & $\min$. & & 1.6 & $\%$ & $(50 \%)$ & & \\
\hline Stage N3 (delta) & 50.0 & $\min$. & & 7.7 & $\%$ & $(15 \%)$ & & \\
\hline Stage $\mathbf{R}$ (REM) & 55.0 & $\min$. & & 9.4 & $\%$ & $(20 \%)$ & & \\
\hline Stage W (wake) & 22.5 & $\min$. & & 8.0 & $\%$ & $(10 \%)$ & & \\
\hline Average 02 while a & & & 97 & $\%$ & Mini & mum 02 value: & 90 & $\%$ \\
\hline Average 02 while is & & & 96 & $\%$ & & & & \\
\hline Average 02 while i & n-REM: & & 96 & $\%$ & & & & \\
\hline
\end{tabular}

Unit Requested:

Mask Type:

CPAP M-Series/Heated Humidification

Mask Size: Comfort Select

Ramp Time:

Small

Oxygen Therapy:

20 Minutes

Not Required

Oxygen Flow:

Not Required

Chinstrap:

Yes

Lowest Pressure Tried: $\quad 5$

Highest Pressure Tried: 12

cm H2O

Final Optimal Pressure:

9

cm H2O

cm H2O 


\title{
Sleep Medicine Specialists
}

\author{
1169 Eastern Parkway, Suite \#3357 \\ Louisville, Kentucky 40217 \\ Phone 502.454.0755 \\ Fax 502.454.3497
}

\section{CPAP Titration}

Re:

Study date:

CPAP was titrated to a pressure of $9 \mathrm{~cm} / \mathrm{H} 20$ with no significant OSA observed, and the patient tolerated CPAP well. It is recommended that the patient follow up with Dr. David winslow within one month for CPAP compliance therapy.

Physician Signature _ 


\section{Appendix I}

\section{CPAP Compliance Report}

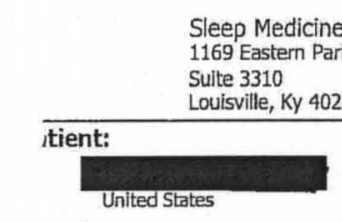

Referring Physician:

PCP:

Clinician:
Device: REMstar Auto M Series with A-Flex ( 510M)

\section{Compliance Details - Full Report}

Compliance Information $7 / 1 / 2008-7 / 7 / 2008$

\section{8}

Patient ID

Home Phone

Age
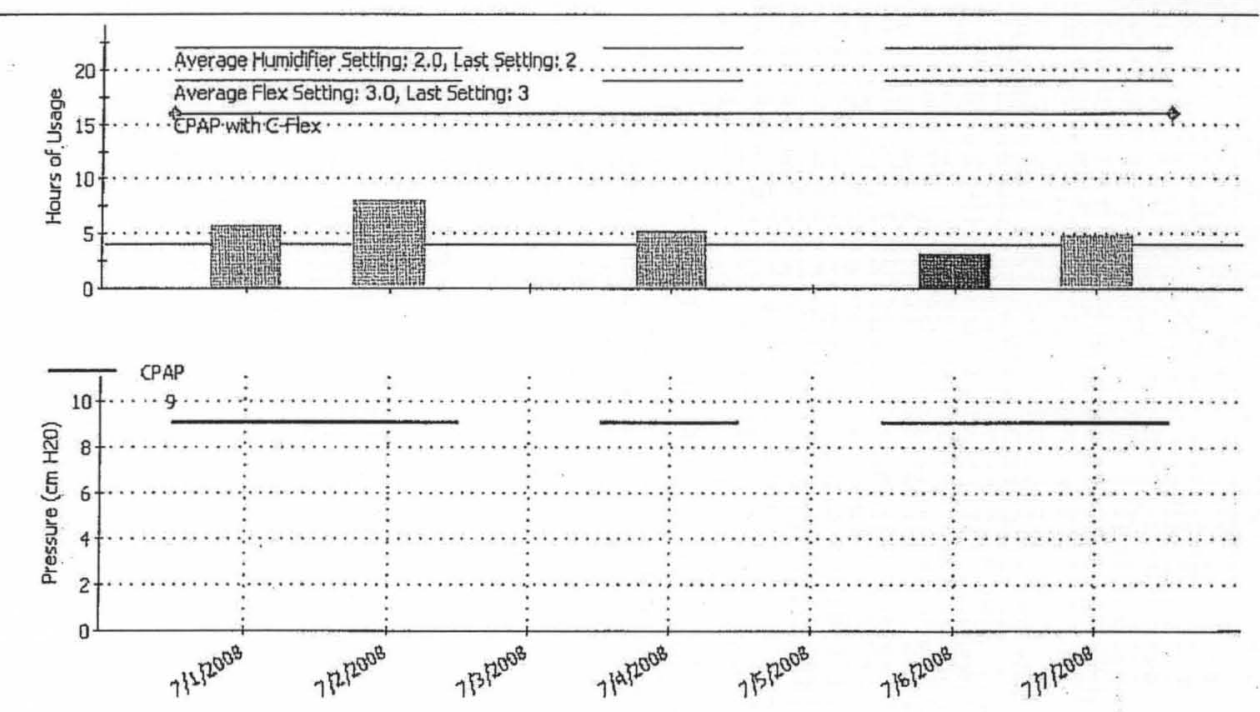


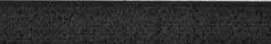

\section{Patterns of use}

July, 2008

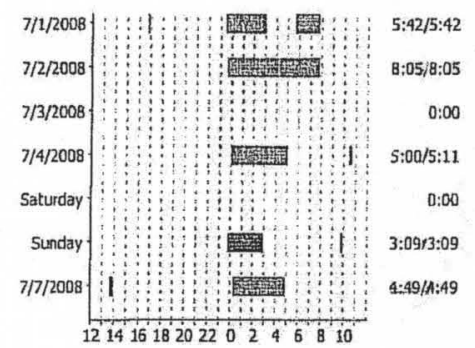

Q. Printed by Encore Pro - Version: 1.8.65

RESPIRONICS

Page 2, of 9 
Appendix J

Visit Schedule of Procedures

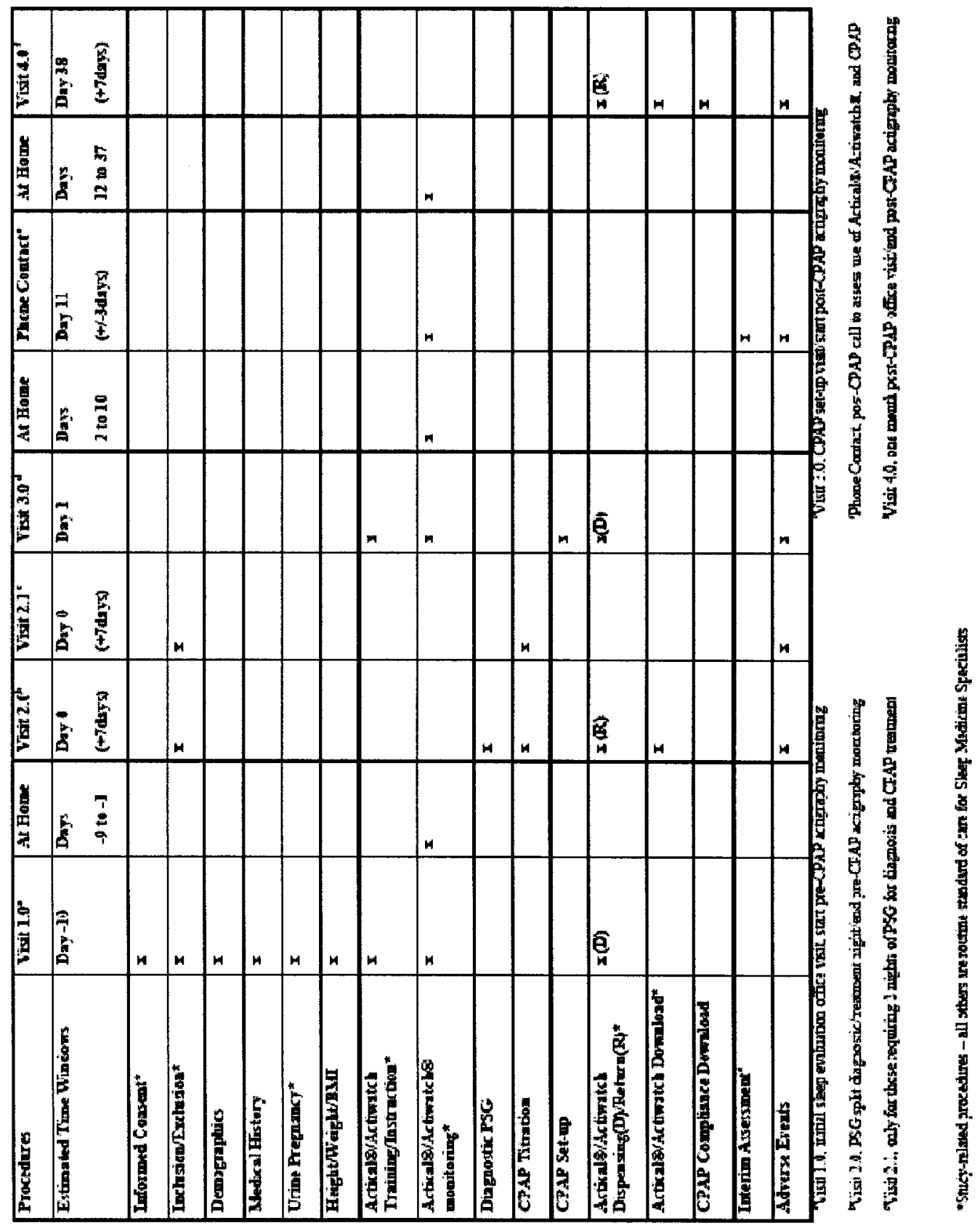




\section{Appendix K}

\section{Data Collection Forms}

Visit 1.0 (Initial Sleep Evaluation, Pre-CPAP)

\section{Date of Visit:}

\section{Informed Consent Process:}

Date of Informed Consent: Version:

Study procedures, risks, benefits, and other relevant study information were explained to the subject. Subject was allowed to ask questions and all questions were answered completely by assigned study staff. Subject was given a copy of the signed ICF. The subject signed the ICF prior to the performance of any study-related procedures.

\begin{tabular}{|l|l|l|}
\hline Y & N & Checklist: \\
\hline & & Subject is able to read, write, and comprehend the English language? \\
\hline & & Subject read and verbalized understanding of the informed consent? \\
\hline & Adequate time was allowed? \\
\hline & & Subject signed and dated the informed consent? \\
\hline & & A copy was provided to subject for his/her records? \\
\hline
\end{tabular}

Individual conducting consent process:

Date:

Date of Birth:

Age:

Gender: Male / Female

\section{Race:}

Caucasian descent

Asian descent

African descent

American Indian or Alaskan Native

Native Hawaiian or Other Pacific Islander

Other (specify): 


\section{Marital Status:}

Single

Married

Divorced

Widowed

Other:

Education level: (highest level obtained)

$<12$ years

High School Diploma (12 years)

Technical/Vocational School

College/University Undergraduate (14 years)

College/University Undergraduate (16 years)

College/University Graduate (18 years)

College/University Post-Graduate (18+ years)

\section{Work Status:}

Unemployed

Part-time

Full-time

Disabled

\section{Work Schedule:}

Day shift

Evening shift

Night shift

Swing shift

\section{Sleep Schedule:}

Usual bedtime: Usual time of awakening:

\section{Medical History:}

Hypertension

Myocardial infarction

Heart failure

Cerebrovascular accident

Dyslipidemia

Diabetes

Depression

Other (specify):

Any acute, unstable, or untreated medical conditions?

Yes:

No

Height: in . $\mathrm{cm}$

Weight: _. Ibs

-.. $\mathrm{kg}$

BMI: $\mathrm{kg} \div$ $m)^{2}=$ (weight $\mathrm{kg}) \div$ [height $(\mathrm{m})]^{2}$ 
Child-bearing Potential: Female of childbearing potential?

Yes/No:

N/A:

Urine Pregnancy Test Results: Positive/Negative N/A

Expiration/Lot \#:

Eligibility Review: (all must be marked yes, "Y", for study inclusion)

1) male or female;

2) adults age 18 to 65 ;

3) able to speak, read and write the English language;

4) overweight or obese (BMI $\geq 25$ );

5) initially present with signs and symptoms consistent with OSA, which is newly and diagnostically confirmed with an $\mathrm{AHI} \geq 5$ as determined by PSG;

6) require CPAP therapy (physician-prescribed);

7) maintain traditional work hours (no night shift work);

8) go to bed between 9:00 pm and 1:00 am, allowing 6 to 9 hours of sleep time;

9) no known acute, unstable, or untreated medical conditions; and

10) not pregnant or planning to become pregnant.

\section{Actigraphy:}

Subject instructed on the use of the Actiwatch $®$ and Actical $\circledast$ devices:

$$
\text { Yes/No }
$$

Actiwatch ${ }^{\circledR}$ dispensed:

Actical ${ }^{\circledR}$ dispensed:

Subject asked to wear both wrist devices 24 hours a day (without removing) for ten days prior to their scheduled diagnostic PSG: Yes/No

Diagnostic PSG scheduled per SMS clinical staff:

Yes: Date of PSG:

No: Explain: 
Visit 2.0/2.1 (Diagnostic PSG/CPAP Titration)

\section{Date of Visit:}

PSG:

Split diagnostic/CPAP titration

Diagnostic only

Apnea/Hypopnea Index:

CPAP Titration PSG scheduled per SMS clinical staff:

Yes: Date of CPAP Titration PSG:

No: Explain:

N/A

CPAP Pressure:

$\mathrm{cm} \mathrm{H} 2 \mathrm{O}$

Eligibility Review: (must be marked yes, "Y", for study inclusion)

- OSA diagnostically confirmed with an $\mathrm{AHI} \geq 5$ as determined by PSG;

- require CPAP therapy (physician-prescribed);

\section{Actigraphy:}

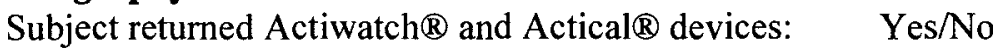

\section{Actiwatch@ data downloaded: start date}

/end date

Day 1: Total sleep minutes: Total wake minutes:

Day 2: Total sleep minutes: Total wake minutes:

Day 3: Total sleep minutes: Total wake minutes:

Day 4: Total sleep minutes: Total wake minutes:

Day 5: Total sleep minutes: Total wake minutes:

Day 6: Total sleep minutes: Total wake minutes:

Day 7: Total sleep minutes: Total wake minutes:

Day 8: Total sleep minutes: Total wake minutes:

Day 9: Total sleep minutes: Total wake minutes:

Day 10: Total sleep minutes: Total wake minutes: 
Day 1: Total EE:

Day 2: Total EE:

Day 3: Total EE:

Day 4: Total EE:

Day 5: Total EE:

Day 6: Total EE:

Day 7: Total EE:

Day 8: Total EE:

Day 9: Total EE:

Day 10: Total EE:

Adverse Events: (related to study participation and/or procedures)

Yes:

No

CPAP set-up scheduled per SMS clinical staff:

\section{Comments:}

Signature of individual completing source documents

Date 
Visit 3.0 (CPAP Set-Up, Post-CPAP)

Date of Visit:

Actigraphy:

Subject instructed on the use of the Actiwatch $\circledast$ and Actical $\circledast$ devices:

Yes/No

Actiwatch $\circledast$ dispensed:

Actical $\otimes^{8}$ dispensed:

Subject asked to wear both wrist devices 24 hours a day (without removing) for approximately 40 days starting with the first day of CPAP use at home: Yes/No

CPAP:

CPAP compliance card included with CPAP equipment: Yes/No

Adverse Events: (related to study participation and/or procedures)

Yes:

No

One Month Follow-Up visit scheduled per SMS clinical staff:

Comments:

Signature of individual completing source documents

Date 


\section{Phone Contact (Post-CPAP, At Home)}

Date of Phone Contact:

\section{Actigraphy:}

Assess use of Actiwatch $\circledast$ and Actical ${ }^{\circledR}$ devices at home: Yes/No

Comments:

\section{CPAP:}

Assess use of CPAP at home: Yes/No

Comments:

Adverse Events: (related to study participation and/or procedures)

Yes:

No

Confirm One Month Follow-Up visit scheduled per SMS clinical staff:

\section{Comments:}


Visit 4.0 (One month Post-CPAP)

Date of Visit:

\section{Actigraphy:}

Subject returned Actiwatch $\circledast$ and Actical $B$ devices: $\quad$ Yes $/$ No

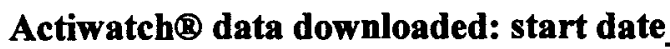

/end date

Day 1: Total sleep minutes: Total wake minutes:

Day 2: Total sleep minutes: Total wake minutes:

Day 3: Total sleep minutes: Total wake minutes:

Day 4: Total sleep minutes: Total wake minutes:

Day 5: Total sleep minutes: Total wake minutes:

Day 6: Total sleep minutes: Total wake minutes:

Day 7: Total sleep minutes: Total wake minutes:

Day 8: Total sleep minutes: Total wake minutes:

Day 9: Total sleep minutes: Total wake minutes:

Day 10: Total sleep minutes: Total wake minutes:

Day 11: Total sleep minutes: Total wake minutes:

Day 12: Total sleep minutes: Total wake minutes:

Day 13: Total sleep minutes: Total wake minutes:

Day 14: Total sleep minutes: Total wake minutes:

Day 15: Total sleep minutes: Total wake minutes:

Day 16: Total sleep minutes: Total wake minutes:

Day 17: Total sleep minutes: Total wake minutes:

Day 18: Total sleep minutes: Total wake minutes:

Day 19: Total sleep minutes: Total wake minutes: 
Day 20: Total sleep minutes: Total wake minutes:

Day 21: Total sleep minutes: Total wake minutes:

Day 22: Total sleep minutes: Total wake minutes:

Day 23: Total sleep minutes: Total wake minutes:

Day 24: Total sleep minutes: Total wake minutes:

Day 25: Total sleep minutes: Total wake minutes:

Day 26: Total sleep minutes: Total wake minutes:

Day 27: Total sleep minutes: Total wake minutes:

Day 28: Total sleep minutes: Total wake minutes:

Day 29: Total sleep minutes: Total wake minutes:

Day 30: Total sleep minutes: Total wake minutes:

Day 31: Total sleep minutes: Total wake minutes:

Day 32: Total sleep minutes: Total wake minutes:

Day 33: Total sleep minutes: Total wake minutes:

Day 34: Total sleep minutes: Total wake minutes:

Day 35: Total sleep minutes: Total wake minutes:

Day 36: Total sleep minutes: Total wake minutes:

Day 37: Total sleep minutes: Total wake minutes:

Day 38: Total sleep minutes: Total wake minutes:

Day 39: Total sleep minutes: Total wake minutes:

Day 40: Total sleep minutes: Total wake minutes: 
Day 1: Total EE:

Day 2: Total EE:

Day 3: Total EE:

Day 4: Total EE:

Day 5: Total EE:

Day 6: Total EE:

Day 7: Total EE:

Day 8: Total EE:

Day 9: Total EE:

Day 10: Total EE

Day 11: Total EE

Day 12: Total EE

Day 13: Total EE:

Day 14: Total EE:

Day 15: Total EE:

Day 16: Total EE:

Day 17: Total EE:

Day 18: Total EE:

Day 19: Total EE:

Day 20: Total EE:
Day 21: Total EE:

Day 22: Total EE:

Day 23: Total EE:

Day 24: Total EE:

Day 25: Total EE:

Day 26: Total EE:

Day 27: Total EE:

Day 28: Total EE:

Day 29: Total EE:

Day 30: Total EE:

Day 31: Total EE:

Day 32: Total EE:

Day 33: Total EE:

Day 34: Total EE:

Day 35: Total EE:

Day 36: Total EE:

Day 37: Total EE:

Day 38: Total EE:

Day 39: Total EE:

Day 40: Total EE: 
CPAP:

Subject provided CPAP compliance card: Yes/No

CPAP compliance data downloaded: start date /end date

Day 1: Hours of use:

Day 2: Hours of use:

Day 3: Hours of use:

Day 4: Hours of use:

Day 5: Hours of use:

Day 6: Hours of use:

Day 7: Hours of use:

Day 8: Hours of use:

Day 9: Hours of use:

Day 10: Hours of use:

Day 11: Hours of use:

Day 12: Hours of use:

Day 13: Hours of use:

Day 14: Hours of use:

Day 15: Hours of use:

Day 16: Hours of use:

Day 17: Hours of use:

Day 18: Hours of use:

Day 19: Hours of use:

Day 20: Hours of use:
Day 21: Hours of use:

Day 22: Hours of use:

Day 23: Hours of use:

Day 24: Hours of use:

Day 25: Hours of use:

Day 26: Hours of use:

Day 27: Hours of use:

Day 28: Hours of use:

Day 29: Hours of use:

Day 30: Hours of use:

Day 31: Hours of use:

Day 32: Hours of use:

Day 33: Hours of use:

Day 34: Hours of use:

Day 35: Hours of use:

Day 36: Hours of use:

Day 37: Hours of use:

Day 38: Hours of use:

Day 39: Hours of use:

Day 40: Hours of use:

Adverse Events: (related to study participation and/or procedures)

Yes:

No 
End of Study

Comments:

Signature of individual completing source documents

Date 


\title{
CURRICULUM VITAE
}

\section{Pamela Ann McCullough, M.S.N., A.P.R.N.}

\author{
1169 Eastern Parkway, Suite 2243 \\ Louisville, Kentucky 40217 \\ 502-479-1217 \\ 502-897-2217 \\ pmccullough@kyresearchgroup.com
}

\section{$\underline{\text { Education }}$}

2008-present Doctoral Candidate

School of Nursing, University of Louisville, Louisville, Kentucky

2006-2008 Ph.D. Student

School of Nursing, University of Louisville, Louisville, Kentucky

1997

M.S.N., Adult Nurse Practitioner

School of Nursing, University of Louisville, Louisville, Kentucky

1991

B.S.N.

School of Nursing, University of Louisville, Louisville, Kentucky

\section{Certifications \& Licensure}

Kentucky Registered Nurse, License \#1068451

Kentucky Advanced Registered Nurse Practitioner, Registration \#3002565

ACRP Certified Clinical Research Coordinator, 2004 - present

Basic Cardiac Life Support, 2004 - present

\section{Professional Employment}

2005-present Director of Clinical Research

Kentucky Research Group

Louisville, Kentucky

2000-2005 Nurse Practitioner (clinical/research)

Sleep Medicine Specialists/Chest Medicine Associates

Louisville, Kentucky/Frankfort, Kentucky 
1998-2000 Nurse Practitioner

Sleep Disorders Center

Audubon Hospital, Louisville, Kentucky

1997-1998 Registered Nurse/Nurse Practitioner (part-time)

Sleep Lab

Baptist Hospital East, Louisville, Kentucky

Registered Nurse/Nurse Practitioner (part-time)

Hospital rounds/Office clinic

Pulmonary and Internal Medicine Associates, Louisville, Kentucky

1994-1997 Registered Nurse - Weekend Pulmonary Rounds (part-time)

Physician group: Chest Medicine Associates

Audubon Hospital, Louisville, Kentucky

1992-1998 Nurse Clinician/Registered Nurse (full-time)

Sleep Disorders Center

Audubon Hospital, Louisville, Kentucky

1991-1992 Staff Registered Nurse/Charge Nurse

Cardiopulmonary/Oncology Unit

Audubon Hospital, Louisville, Kentucky

1988-1991 Nurse Extern/Patient Care Assistant

Nursing Pool

Audubon Hospital, Louisville, Kentucky

\section{Publications}

Winslow, D. H., Bowden, C. H., DiDonato, K., McCullough, P. A., Day, W. W. (2010). A randomized, double-blind, placebo-controlled, parallel-group study of Phentermine/Topiramate CR (VI-0521) for the treatment of obstructive sleep apnea/hypopnea syndrom in obese adults [Abstract]. Sleep Abstract Supplement, Abstract No. 0475.

Roth, T. \& McCullough, P.A. (2005). Understanding and managing insomnia. AANP Monograph. Wrightstown, PA: Medical Communications Media. 
McCullough, P.A. (1999). Sleep and pregnancy. University of Louisville, Perinatal Coordinating Center News, 27(9).

McCullough, P. A. (1998). Obstructive sleep apnea: does level of severity correlate with level of depression? Sleep Abstract Supplement.

McCullough, P.A. (1988). Relating OSA and depression. The Journal for Respiratory Care Practitioners/RT Magazine, 29-31.

\section{Presentations}

(Speaker, Invited)

McCullough, P.A. Nursing Journal Review. Kentucky Sleep Society Annual Sleep Medicine Conference, Louisville, KY. 2007 \& 2008.

(Speaker, Invited)

McCullough, P.A. How Well Do You Sleep? Kentucky Coalition of Nurse Practitioners and Nurse Midwives Annual Conference, Louisville, KY. 2001.

(Speaker, Invited)

McCullough, P.A. Optimizing Your Sleep. Speaking of Women's Health Conference, Louisville, KY. 2001.

(Poster) March 1999.

McCullough, P.A. OSA and depression. Narcolepsy Conference, Chicago, IL.

(Poster)

McCullough, P.A. Obstructive sleep apnea: does level of severity correlate with level of depression? Associated Professional Sleep Society $12^{\text {th }}$ Annual Meeting, New Orleans, LA. June 1998.

\section{Professional Organizations}

Association of Clinical Research Professionals

Kentucky Coalition of Nurse Practitioners and Nurse Midwives

American Academy of Sleep Medicine

Kentucky Sleep Society

\section{Continuing Education}

1994-present Associated Professional Sleep Society Annual Conference

1999-present Kentucky Sleep Society Annual Meeting

2000-2006 Annual KCNPNM Conference 
2004 Annual Association of Clinical Research Professionals Meeting

2002 Investigator Training for Medical Research. University of Rochester School of Medicine \& Dentistry, Strong Memorial Hospital, and Western Institutional Review Board. Chicago, IL.

2000 Sleep-Disordered Breathing and CHF, Louisville, KY.

2000 Pediatric Conference for the Technologist, Louisville, KY.

1999 Workshop on the Sleep Needs, Patterns and Difficulties of Adolescents. Washington, D.C.

1999 Narcolepsy Conference, Chicago, IL.

1997-1998 Annual KCNPNM Conference

1994 Regional Polysomnographic Technologist Conference, Chicago, IL.

1993 Polysomnographic Physician Course. The Sleep Disorders Center of Alabama. Birmingham, AL.

\section{$\underline{\text { Research Experience }}$}

Industry-Sponsored Pharmaceutical Research Role: Sub-Investigator/Research Coordinator

2011-present Boehringer Ingelheim 1245.20: A phase III randomised, double-blind, placebo-controlled parallel group efficacy and safety study of BI 10773 and sitagliptin administered orally over 24 weeks, in drug naïve patients with type 2 diabetes mellitus and insufficient glycaemic control despite diet and exercise. 2011 - Ongoing

Sanofi Aventis EFC6058: TERACLESA multi-center double-blind parallel-group placebo-controlled study of the efficacy and safety of teriflunomide in patients with relapsing multiple sclerosis who are treated with interferon-beta 2011 - Ongoing.

Novartis ACZ885H Study: 2361: A Randomized, Double-Blind, ActiveControlled Study of Canakinumab (ACZ885) Pre-Filled Syringes or Reconstituted Lyophilizate Versus Triamcinolone Acetonide for Treating Acute Gouty Arthritis Flares in Frequently Flaring Patients.2011-Ongoing. Merck 029: A Phase III, Multicenter, Randomized, Double-Blind, Placebo-Controlled, Parallel Group Study to Evaluate the Safety and Efficacy of MK-4305 in Patients with Primary Insomnia. 2011 - Ongoing. 
2010-present Forest LAS-MD-35: Long Term Safety and Tolerability of Aclidinium Bromide When Administered Twice Daily to Patients with Moderate to Severe Chronic Obstructive Pulmonary Disease. 2010 - Ongoing. Cephalon C10953/4030: A Randomized, Double-Blind, PlaceboControlled Study to Evaluate the Efficacy and Tolerability of Armodafinil Treatment (150mg) in Improving Clinical Condition Late in the Shift and in Improving Functional and Patient-Reported Outcomes in Adult Patients With Excessive Sleepiness Associated With Shift Work Disorder. 2010.

Eli Lilly H9X-MC-GBDC: The Impact of LY2189265 Versus Metformin on Glycemic Control in Early Type 2 Diabetes Mellitus (AWARD-3: Assessment of Weekly Adminst $\underline{R}$ ation of LY2189265 in Diabetes-3) 2010 - Ongoing

Eli Lilly H9X-MC-GBDN: The Effect of LY2189265 on Blood Pressure and Heart Rate, as Assessed by Ambulatory Blood Pressure Monitoring, in Patients with Type 2 Diabetes Mellitus. 2010 - Ongoing.

Boehringer Ingelheim 205.452: A Randomized, Active-Controlled, Double-Blind, Double-Dummy, Parallel Group Design, Multi-Center Trial to Compare the Efficacy and Safety of $2.5 \mathrm{mg}$ and $5 \mathrm{mg}$ Tiotropium Inhalation Solution Delivered by the Respimat Inhaler with Tiotropium Inhalation Capsules 18mg Delivered by the HandiHaler. 2010 - Ongoing Philips Respironics: BiPAP autoSV Advanced in Central Apnea Patients 2010 - Ongoing.

2009-2010 Takeda AD4833_402: A Phase \$, Randomized, Double-Blind, PlaceboControlled Study to Evaluate the Effect of Pioglitazone Compared to Placebo on Bone Metabolism in Impaired Fasting Glucose, Postmenopausal Women for 1 Year of Treatment 2009 - Ongoing. Pfizer A0081186: Randomized, double-blind 12-month Study of Pregabalin in Subjects with Restless Legs Syndrome. 2009-2010. Ventus C009-Aero: A prospective, multicenter parallel group, shamcontrolled, randomized, double-blinded clinical trial to compare the safety and effectiveness of the Provent Professional Sleep Apnea Therapy device to sham for the non-invasive treatment of obstructive sleep apnea. 20092010.

Sanofi-Aventis EFC3014 GetGoalM: A Randomized, Double-Blind, Placebo-Controlled Parallel-Group, Multicenter, 24 week Study Followed by an Extention Assessing the Efficacy and Safety of AVE0010 on Top of Metformin in Patients with Type-2 Diabetes not Adequately Controlled with Metformin. 2009-Ongoing.

Cephalon C10953-3067/ES/MN: A 12-week, Randomized, DoubleBlind, Placebo-Controlled, Parallel-Group, Fixed-Dosage, Study to Evaluate the Efficacy and Safety of Armodafinil $(50,150$, and 250 $\mathrm{mg}$ /day) as Treatment for Patients With Excessive Sleepiness Associated With Mild to Moderate Closed Traumatic Brain Injury. 2009-2010. 
Ventus C009E-Aero: A prospective, multicenter, single arm, open-label extension study to evaluate the long-term durability of treatment response and safety of the Provent Professional Sleep Apnea Therapy Devices for the non-invasive treatment of obstructive sleep apnea - hypopnea. 2009 2010.

Pfizer A8801015: A Randomized Phase 2, Double-Blind, PlaceboControlled, Multi-Center, Crossover Study of PF-03654745 as a Daily Treatment for Excessive Daytime Sleepiness (EDS) Associated with Narcolepsy. 2009-2010.

Pfizer A0081165: Effects of Pregabalin on Sleep Maintenance in Subjects with Fibromyalgia Syndrome and Sleep Maintenance Disturbance: Randomized, Placebo-Controlled, 2-way Crossover Polysomnography Study. 2009-2010.

Merck 009: A Phase III, Multicenter, Randomized, Double-Blind, Placebo-Controlled, Parallel-Group, Long Term Safety Study of MK-4305 in Patients with Primary Insomnia. 2009 - Ongoing.

2008-2009 Merck 015: A Multicenter, Randomized, Double-Blind, Double-Dummy, Placebo and Modafinil Controlled, 3-Period Crossover, Adaptive Dose Design Study to Determine the Safety and Efficacy of MK-0249 in Treating Excessive Daytime Sleepiness in Patients with Obstructive Sleep Apnea Using Nasal Continuous Positive Airway Pressure (nCPAP) Therapy.

Arena APD125-007: A Randomized, Double-Blind, Placebo-Controlled Subjective Study to Assess the Efficacy of APD125 in Patients with Primary Insomnia Characterized by Difficulty Maintaining Sleep. Pfizer A0081183: Randomized, Double-Blind, 6 Week Study of Pregabalin in Subjects with Restless Leg Syndrome. 2008 - Ongoing. Sanofi EFC6909: A Double-Blind, Randomized, 12-Month, PlaceboControlled, Parallel-Group, Fixed-Dose Study to Evaluate the Efficacy and Safety of AVE5530 25mg/day and AVE5530 50mg/day in Patients with Primary Hypercholesterolemia.

VIVUS OB-204: A Randomized, Double-Blind, Placebo-Controlled, Parallel-Group Study of VI-0521 for the Treatment of Obstructive Sleep Apnea/Hypopnea Syndrome in Obese Adults.

Sanofi EFC10844/ECLIPSE: Efficacy and Safety of Eplivanserin $5 \mathrm{mg} /$ day in Insomnia Characterized by Sleep Maintenance difficulties: A 6-Week, Randomized, Double-Blind, Placebo-Controlled, Polysomnography Study.

Pfizer A9001380: Refinement of Patient Reported Outcomes Instruments in Subjects with Insomnia Characterized by Non Restorative Sleep.

2007-2008 Merck 079: Randomized, Double-Blind, Active-Comparator, Clinical Trial to Study the Efficacy and Safety of MK-0431A for the Treatment of Patients with Type 2 Diabetes Mellitus (T2DM). 
Vanda VP-VEC-162-3104: A Multicenter, Randomized, Double-Blind, Placebo-Controlled, Parallel Study to Investigate the Efficacy and Safety of VEC-162 (20mg/day and $50 \mathrm{mg} /$ day) in the Treatment of Primary Insomnia.

Sanofi-Aventis LTE6673/SAMS: Efficacy and Safety of 2mg/day of M100907 on Sleep Maintenance Insomnia with a Sub-Study of the effect of M100907 on Stable Type II Diabetes Mellitus: A One Year, Multicenter, Randomized, Double- Blind, Placebo-Controlled Study.

Pfizer A5351019: A 2-Year, Randomized, Double-Blind, PlaceboControlled Phase 3 Study to Evaluate the Long-Term Efficacy and Safety of CP-945,598 in the Treatment of Obese Subjects.

Organon 176001: A Two-Week, Double-Blind, Placebo-Controlled, Randomized, Parallel Group, Efficacy and Safety, Outpatient Trial with Org 50081 in Patients with Chronic Primary Insomnia.

Organon 176004: Fifty-Two Weeks, Open-Label Extension Trial to Evaluate Safety and Efficacy of Org 50081 in Outpatients with Chronic Primary Insomnia who Completed Clinical Trial Protocol 176001 or 176002.

Takeda 01-06-TL-375-071: Randomization, Double-Blind, PlaceboControlled Study to Assess Whether the Administration of Ramelteon Could Facilitate the Discontinuation of Zolpidem (Ambien) $>10 \mathrm{mg}$ Therapy in Subjects with Chronic Insomnia.

Vivus OB-301: A Phase III, Randomized, Double-Blind, Parallel-Design Study Comparing Multiple Doses of VI-0521 to Placebo and Their Single-Agent Phentermine and Topiramate Constituents for the Treatment of Obesity in Adults.

Sanofi-Aventis EFC6072/Nocturne: Efficacy and Safety of 2mg/day of M100907 on Sleep Maintenance Insomnia: a 6-week, Multi-center, Randomized, Double-Blind, Placebo-Controlled Polysomnographic Study.

Xenoport XP-081: A Randomized, Double-Blind, Placebo-Controlled Dose Response Study to Assess the Efficacy, Safety, and Pharmacokinetics of XP13512 in Patients with Restless Legs Syndrome. Merck V512-003: Validation of Patient Reported Outcome Measures for Influenza Disease for Use in Phase II POC/Phase III Flu Peptide Vaccine Program.

Xenoport XP083: A Randomized, Double-Blind, Active- and Placebo Controlled, Parallel Group Safety Study Assessing Simulated Driving Performance in XP13512-Treated Patients with Restless Legs Syndrome.

2006-2007 Sanofi-Aventis EFC6220: Efficacy and Safety of Eplivanserin 5mg/day on Sleep Maintenance Insomnia: a 6-Week, Multicenter, Randomized, Double-Blind, Placebo-Controlled Study. 
Merck 022-01: A Multicenter, Randomized, Double-Blind, "Factorial" Design Study to Evaluate the Lipid-Altering Efficacy and Safety of MK0524B (dosed as coadministered MK-0524A and Simvastatin Tablets) in Patients With Primary Hypercholesterolemia or Mixed Hyperlipidemia. Novartis CVEA489A2302: An 8-Week, Multicenter, Randomized, Double-Blind, Parallel-Group Study to Evaluate the Efficacy and Safety of the Combination of Valsartan/HCTZ/Amlodipine Compared to Valsartan/HCTZ, Valsartan/Amlodipine, and HCTZ/Amlodipine in Patients With Moderate to Severe Hypertension.

Merck 315: A Double-Blind, Randomized, Parallel, Efficacy Study Evaluating Losartan Potassium Alone or in Combination with Hydrochlorothiazide versus Placebo in Obese Patients with Elevated Systolic and Diastolic Blood Pressure.

XenoPort, Inc. XP055: An Open-Label, 52-Week Extension Study Assessing XP13512 Safety and Efficacy in Patients with Restless Legs Syndrome.

Takeda 01-05-TL-375-067: A Phase2, Double-Blind, Randomized, Placebo-Controlled, Parallel-Group, Multi-Center Proof-of-Concept Study to Evaluate the Safety and Efficacy of Ramelteon Taken in Combination with Doxepin for the Treatment of Subjects with Chronic Insomnia. GlaxoSmithKline 103660: A 12-Week, Multi-Center, Double-Blind, Placebo-Controlled, Parallel Group, Flexible Dose Polysomnography Study of Ropinirole Controlled Release for Restless Legs Syndrome (RLS) in RLS Patients with Sleep Disturbance and Periodic Limb Movements (PLM) During Sleep.

Merck 023: a Worldwide, Multicenter, Double-Blind, Randomized, Parallel Study to Evaluate the Efficacy of MK-0524 to Improve Tolerability of Extended Release Niacin.

XenoPort, Inc. XP052: A Randomized, Double-Blind, PlaceboControlled Study to Assess the Efficacy and Safety of XP13512 in Patients with Restless Legs Syndrome.

Schwarz SP793: An Open-Label Extension Trial to Investigate the Safety and Tolerability of Long-Term Treatment with Transdermal Rotigotine in Subjects with Idiopathic Restless Legs Syndrome.

Sanofi-Aventis LTE6262: Efficacy and Safety of Eplivanserin 5mg/day on Sleep Maintenance Insomnia: a 12-Week Multicenter, Randomized, Double-Blind, Placebo-Controlled Study Followed by an Open Treatment Phase Extension with Eplivanserin for a 40 Week Period.

Merck 022: A Multicenter, Randomized, Double-Blind, "Factorial" Design Study to Evaluate the Lipid-Altering Efficacy and Safety of MK-0524B Combination Tablet in Patients With Primary Hypercholesterolemia or Mixed Hyperlipidemia.

Merck 024: A Multicenter, Randomized, Double-Blind, Parallel Group, 12 Week Study to Evaluate the Efficacy and Safety of MK-0524B Versus Atorvastatin in Patients with Mixed Hyperlipidemia. 
Takeda 01-05-TL-375-068: A Study of the Safety of Ramelteon with Moderate to Severe Chronic Obstructive Pulmonary Disease.

2005-2006 Schwarz SP792: A Multi-Center, Randomized, Double-Blind, PlaceboControlled, Five-Arm Parallel-Group Trial to Investigate the Efficacy and Safety of Four Different Transdermal Doses of Rotigotine in Subjects with Idiopathic Restless Legs Syndrome.

Kyowa 6002-US-201: A Phase 2, Double-Blind, Placebo Controlled, Randomized Parallel-Group, Multicenter Study to Evaluates the Efficacy and Safety of 40mg/day KW-6002 (Istradefylline) in Subjects with Restless Legs Syndrome.

Somaxon SP-0503: A Phase III, Randomized, Double-Blind, Placebo Controlled, Parallel-Group, Multicenter Study to Assess the Long Term Efficacy and Safety of Doxepin HCI in Primary Elderly Insomnia Patients with Sleep Maintenance Difficulties.

Takeda EC302: A Randomized, Double-Blind, Placebo-Controlled Study to Determine the Long-Term Efficacy and Safety of Ramelteon in Adults with Chronic Insomnia.

Cephalon C10953/3046/ES/US: A Short-Term (8-Week) Open-Label Study, Followed by a Long-Term Evaluation, to Assess Patient Reported Outcomes with Armodafinil Treatment (150 to $250 \mathrm{mg} /$ day) for Excessive Sleepiness in Adults with Narcolepsy or Obstructive Sleep Apnea/Hypopnea Syndrome (OSAHS).

2005 GlaxoSmithKline 1001468/205: A 12 Week, Double-Blind, PlaceboControlled, Parallel-Group Study to Assess the Efficacy and Safety of Ropinirole XR (Extended Release) in Patients with Restless Legs Syndrome.

Takeda 01-04-TL-375-041: A Randomized, Double-Blind, Placebo Controlled Study to Assess the Subjective Response to Treatment with Ramelteon (TAK-375) in Adult Subjects With Chronic Insomnia by Utilizing an Interactive Voice Response System (IVRS) for Collecting Diary Data.

Neurocrine NBI-34060-MR-0501: A Phase III, Randomized, DoubleBlind, Placebo Controlled, Polysomnographic Study to Assess the Efficacy and Safety of a Modified Release Formulation of NBI-34060 in Primary Insomnia Patients with Sleep Maintenance Difficulties.

Somaxon SP-0501: A Phase III, Randomized, Double-Blind, Placebo Controlled, Parallel-Group, Multicenter Study to Assess the Efficacy and Safety of Doxepin HCL in Primary Insomnia Patients with Sleep Maintenance Difficulties.

2004-2007 Merck 002-00: A Double-Blind, Randomized, Placebo-Controlled, Multicenter, 30-Night Polysomnographic Study of MK-0928 in Elderly Patients with Primary Insomnia 
Oscient OS-001/FORCE: A Phase IV, Stratified, Randomized, Prospective, Unblinded, Active-Control Trial of Factive Versus Biaxin $\mathrm{XL}$ for the Treatment of Community-acquired Pneumonia and Versus Amoxicillin/clavulante for the Treatment of Acute Bacterial Exacerbation of Chronic Bronchitis.

2004-2006 Merck 004-00: A Double-Blind, Randomized, Placebo-Controlled, Multicenter, 30-night Polysomnographic Study of MK-0928 in Adult Patients with Primary Insomnia. 2004-2006.

Cephalon, Inc. C10953/3024/ES/MN:A 12-Month, Open-Label, Flexible-Dosage (100 to $250 \mathrm{mg} /$ day) Extension Study of the Safety and Efficacy of CEP-10953 in the Treatment of Patients With Excessive Sleepiness Associated With Narcolepsy, Obstructive Sleep Apnea/Hypopnea Syndrome, or Chronic Shift Work Sleep Disorder.

2004-2005 Altana Pharma BY217/M2-023: A Randomized, Controlled Study of Roflumilast (250 mcg and $500 \mathrm{mcg}$ ) Versus Placebo in Patients with Asthma. A 24-Week, Multicenter, Multinational, Double-Blind, Parallel Group Clinical Study.

Cephalon Inc, CEP-10953 C10953/3021/AP/MN: A 12-Week, Randomized, Double-Blind, Placebo-Controlled, Parallel-Group Study to Evaluate the Efficacy and Safety of CEP-10953 (150 and $250 \mathrm{mg} /$ day) as Treatment for Adults With Residual Excessive Sleepiness Associated with Obstructive Sleep Apnea/Hypopnea Syndrome.

2004 Aventis M100907C/2004: A North American, 4-Week, Multicenter, Phase IIB Double-Blind, Placebo-Controlled, Randomized, Multiple Dose, Parallel-Group Study of the Efficacy and Safety of $0.5 \mathrm{mg}, 1.0 \mathrm{mg}$, and $2.0 \mathrm{mg}$ M100907 Tablets in the treatment of Sleep Maintenance Insomnia. Sanofi-Synthelabo LTE5407: Evaluation of the Long-Term Efficacy and Safety of Zolpidem-MR 12.5mg Compared to Placebo, when Both are Administered Over a Long-Term Period "As Needed", in Patients with Chronic Primary Insomnia. (A Randomized, Double Blind, PlaceboControlled, Parallel Group, Multicenter, Phase IIIb Clinical Study). Pfizer A9001243: A Study To Define The Non-Restorative Sleep Population.

GlaxoSmithKline 100013: A 12 Week, Double-Blind, Placebo Controlled, Twice Daily Dosing Study to Assess the Efficacy and Safety of Ropinirole in Patients Suffering from Restless Legs Syndrome (RLS) Requiring Extended Treatment Coverage.

2003-2004 Orphan Medical, Inc. OMC-SXB-22: A Randomized, Double-Blind, Double-Dummy, Placebo-Controlled, Parallel-Group, Multi-Center Trial Comparing the Effects of Orally Administered Xyrem (sodium oxybate) and Modafinil with Placebo in the Treatment of Daytime Sleepiness in Narcolepsy. 
GlaxoSmithKline 101468/249: A 12 Week, Double-Blind, Placebo Controlled, Parallel Group Study to Assess the Efficacy and Safety of Ropinirole in Patients Suffering from Restless Legs Syndrome (RLS).

Orphan Medical, Inc. OMC-SXB-15: A Randomized, Double-Blind, Placebo-Controlled, Parallel-Group, Multi-Center Trial Comparing the Effects of Orally Administered Xyrem (Sodium Oxybate) with Placebo for the Treatment of Narcolepsy.

Orphan Medical, Inc. OMC-SXB-19: An Open Label, Multi-Center Safety Trial studying the Effects of Orally Administered Xyrem (Sodium Oxybate).

Takeda 01-03-TL-375-038: A Phase II Safety Study of TAK-375 in Subjects with Mild to Moderate Chronic Obstructive Pulmonary Disease. Sanofi-Synthelabo EFC5202: Evaluation of the Hypnotic Properties of Zolpidem-MR $12.5 \mathrm{mg}$ and Zolpidem $10 \mathrm{mg}$ Marketed Product Compared to Placebo in Patients with Primary Insomnia. A Double- Blind, Randomized, Placebo-Controlled, Three Way Cross-Over Study.

GlaxoSmithKline 101468/191: A 12 Week, Double-Blind, PlaceboControlled Parallel Group Study to Assess the Efficacy, Safety and Tolerability of Ropinirole in Subjects with Restless Legs Syndrome (RLS) Suffering from Periodic Leg Movements of Sleep (PLMS).

Sanofi-Synthelabo EFC4529: Comparison of Efficacy and Safety of Zolpidem-MR $12.5 \mathrm{mg}$ and Placebo in Patients with Primary Insomnia. A Double-Blind, Randomized, Placebo-Controlled, Parallel Group Study. Neurocrine NBI34060-MR-0212: A Phase III, Randomized, DoubleBlind, Placebo-Controlled, Parallel-Group, Multicenter Study to Assess the Efficacy and Safety of a Modified Release Formulation of NBI-34060 in Elderly Primary Insomnia Patients with Sleep Maintenance Difficulties.

Takeda 375-022: A phase III, Open-Label, Fixed Dose Study to Determine the Safety of Long-Term Administration of TAK-375 in Subjects with Chronic Insomnia.

GlaxoSmithKline 101468/243: A 52 Week, Open-Label Extension Study of the Long-term safety and tolerability of Ropinirole in Subjects with Restless Legs Syndrome (RLS).

2001-2002 Sepracor 190-047: A Randomized, Double-Blind, Placebo-Controlled Parallel, Two-Week Objective Efficacy and Safety Study of Esopiclone in Elderly Subjects with Primary Insomnia.

Takeda 375-005: An Efficacy, Safety, and Dose Response Study of TAK-375 in Subjects with Chronic Insomnia.

2000 Sepracor 190-046: A Randomized Double-Blind, Placebo-Controlled Parallel Study of the Efficacy and Safety of (S)-Zopiclone in the Treatment of Adult Subjects with Primary Insomnia. 